\title{
Newton Polyhedra of Discriminants of Projections
}

\author{
A. Esterov
}

Received: 11 January 2009 / Revised: 30 November 2009 / Accepted: 10 January 2010 /

Published online: 6 February 2010

(C) Springer Science+Business Media, LLC 2010

\begin{abstract}
For a system of polynomial equations, whose coefficients depend on parameters, the Newton polyhedron of its discriminant is computed in terms of the Newton polyhedra of the coefficients. This leads to an explicit formula (involving Euler obstructions of toric varieties) in the unmixed case, suggests certain open questions in general, and generalizes a number of similar known results (Gelfand et al. in Discriminants, resultants, and multidimensional determinants. Birkhäuser, Boston, 1994; Sturmfels in J. Algebraic Comb. 32(2):207-236, 1994; McDonald in Discrete Comput. Geom. 27:501-529, 2002; Gonzalez-Perez in Can. J. Math. 52(2):348-368, 2000; Esterov and Khovanskii in Funct. Anal. Math. 2(1), 2008).
\end{abstract}

Keywords Discriminant - Newton polyhedron · Mixed fiber polyhedron · Mixed volume $\cdot$ Elimination theory $\cdot$ Euler obstruction $\cdot$ Dual defectiveness $\cdot$ Cayley trick

\section{Introduction}

Let $F_{0}, \ldots, F_{l}$ be Laurent polynomials on the complex torus $(\mathbb{C} \backslash 0)^{k}$, whose coefficients are Laurent polynomials on the parameter space $(\mathbb{C} \backslash 0)^{n}$. Consider the set $\Sigma \subset(\mathbb{C} \backslash 0)^{n}$ of all values of the parameter, such that the corresponding system of polynomial equations $F_{0}=\cdots=F_{l}=0$ defines a singular set in $(\mathbb{C} \backslash 0)^{k}$. In most cases (see below for details), the closure of $\Sigma$ is a hypersurface, and its defining equation is called the discriminant of $F_{0}=\cdots=F_{l}=0$.

In this paper, we compute the Newton polyhedron of the discriminant in terms of Newton polyhedra of the coefficients of the polynomials $F_{0}, \ldots, F_{l}$. The answer is known in many special cases, and we give a number of references as examples of

Partially supported by RFBR-JSPS-06-01-91063, RFBR-07-01-00593, and INTAS-05-7805 grants.

\footnotetext{
A. Esterov $(\varangle)$

Laboratoire J.-A. Dieudonne, Universite de Nice-Sophia Antipolis Parc Valrose, 06108 Nice Cedex 02, France

e-mail: esterov@mccme.ru
} 
various approaches to this problem: the universal special case for $l=0$ and $l=k$ was studied in $[14,25]$, and [6] (universal case means that $(\mathbb{C} \backslash 0)^{n}$ parameterizes all collections of polynomials $F_{0}, \ldots, F_{l}$, whose monomials are contained in a given finite set of monomials), the general case for $l=k-1$, for $l=k-1=0$, and for $l=k$ was studied in [15, 22], and [13].

To formulate the answer in general, we need the following notation: we denote the Minkowski sum $\{a+b \mid a \in A, b \in B\}$ of polyhedra $A$ and $B$ by $A+B$, and denote the mixed fiber polyhedron of the polyhedra $\Delta_{0}, \ldots, \Delta_{l}$ in $\mathbb{R}^{n} \oplus \mathbb{R}^{k}$ by the monomial $\Delta_{0} \cdots \Delta_{l}$ (it is a certain polyhedron in $\mathbb{R}^{k}$, see $[11,13,23]$, or Definition 2.11). To a polyhedron $A$ and its face $B$ we associate its Euler obstruction $e^{B, A} \in \mathbb{Z}$, whose combinatorial definition is given in Sect. 2.5, and whose geometrical meaning is $(-1)^{\operatorname{dim} A-\operatorname{dim} B}$ times the Euler obstruction of the toric variety of $A$ at its orbit, corresponding to the face $B$ (see also [20] and a remark at the end of Sect. 5.4).

Considering $F_{i}$ as a polynomial on $(\mathbb{C} \backslash 0)^{k}$ with polynomial coefficients, denote its Newton polyhedron by $A_{i} \subset \mathbb{R}^{k}$; considering the same $F_{i}$ as a polynomial on $(\mathbb{C} \backslash 0)^{n} \times(\mathbb{C} \backslash 0)^{k}$ with complex coefficients, denote its Newton polyhedron by $\Delta_{i} \subset \mathbb{R}^{n} \oplus \mathbb{R}^{k}$. Denote the preimage of a face $A^{\prime} \subset A_{i}$ under the natural projection $\Delta_{i} \rightarrow A_{i}$ by $\Delta_{i}\left(A^{\prime}\right)$. For simplicity, we assume here that $A_{0}=\cdots=A_{l}=A$, and pairwise differences of points of $A \cap \mathbb{Z}^{k}$ generate $\mathbb{Z}^{k}$.

Theorem If $F_{0}, \ldots, F_{l}$ are generic polynomials with Newton polyhedra $\Delta_{0}, \ldots, \Delta_{l}$, then the Newton polyhedron of the discriminant equals

$$
\mathcal{N}=\sum_{A^{\prime} \subset A} e^{A^{\prime}, A} \cdot \sum_{\substack{a_{0}>0, \ldots, a_{l}>0 \\ a_{0}+\cdots+a_{l}=\operatorname{dim} A^{\prime}+1}} \Delta_{0}\left(A^{\prime}\right)^{a_{0}} \cdots \Delta_{l}\left(A^{\prime}\right)^{a_{l}},
$$

where $A^{\prime}$ runs over all faces of $A$ of dimension $l$ and greater, including $A^{\prime}=A$.

More precisely, if the polyhedron $\mathcal{N}$ consists of one point, then the discriminant set $\Sigma$ has no codimension 1 components; otherwise, the closure of $\Sigma$ is a hypersurface, and the Newton polyhedron of its equation equals $\mathcal{N}$.

The word "generic" means that collections $F_{0}, \ldots, F_{l}$, for which the statement is true, form a dense set in the space of all collections with Newton polyhedra $\Delta_{0}, \ldots, \Delta_{l}$. Note that coefficients $e^{A^{\prime}, A}$ may be negative, and the formula above involves subtraction of polyhedra. The difference of polyhedra $P$ and $Q$ is by definition the solution of the equation $Q+X=P$, which is always unique if it exists; see the end of Sect. 6.6 for details and related computability questions.

The result that we actually prove is somewhat more general than the theorem above in the following sense. Firstly, together with the Newton polyhedron, we describe the leading coefficients of the discriminant (i.e., the coefficients of monomials in the boundary of the Newton polyhedron) in terms of leading coefficients of the polynomials $F_{0}, \ldots, F_{l}$ (see Theorem 4.3 for $l=k$, Proposition 5.11 for $l=0$, and Theorem 6.4, which reduces the general case to $l=0$ ). Secondly, we do not assume that $A_{0}=\cdots=A_{l}$. Thirdly, we prefer a slightly more general context throughout the paper (see [15] for motivation): instead of polynomials on $(\mathbb{C} \backslash 0)^{n}$, we consider analytic 
functions on an arbitrary affine toric variety. Nevertheless, all our results and proofs can be translated back into the global setting, word by word, substituting germs of analytic functions on affine toric varieties with Laurent polynomials on complex tori, unbounded polyhedra with bounded ones, and the local version of the elimination Theorem 4.3 with its global version [13]. For instance, the theorem above is exactly the global version of Theorem 6.10 with $A_{0}=\cdots=A_{l}$. We illustrate it with an example in Sect. 6.6.

Denote the Newton polyhedron of the discriminant of $F_{0}=\cdots=F_{l}=0$ by $\mathcal{N}\left(\Delta_{0}, \ldots, \Delta_{l}\right)$ for generic equations $F_{0}, \ldots, F_{l}$ with given Newton polyhedra $\Delta_{0}, \ldots, \Delta_{l}$. Theorem 6.10 leads to a certain relation between Newton polyhedra of discriminants (higher additivity, see Sect. 6.4 for details), provided that the Newton polyhedra $A_{0}, \ldots, A_{l}$ are similar to each other and large enough:

$$
\begin{aligned}
\mathcal{N}\left(\Delta_{0}+\Delta_{1}, \Delta_{2}, \ldots, \Delta_{l}\right) & \\
= & \sum_{\mu=1}^{\infty} \mathcal{N}(\underbrace{\Delta_{0}^{\Delta_{0}, \ldots, \Delta_{0}}}_{\mu}, \underbrace{\Delta_{1}, \ldots, \Delta_{1}}_{\mu-1}, \Delta_{2}, \ldots, \Delta_{l}) \\
& +\mathcal{N}(\underbrace{\Delta_{0}^{\Delta_{0}, \ldots, \Delta_{0}}}_{\mu-1}, \underbrace{\Delta_{1}, \ldots, \Delta_{1}}_{\mu}, \Delta_{2}, \ldots, \Delta_{l}) \\
& +2 \mathcal{N}(\underbrace{\Delta_{0}, \ldots, \Delta_{0}}_{\mu}, \underbrace{\Delta_{1}, \ldots, \Delta_{1}}_{\mu}, \Delta_{2}, \ldots, \Delta_{l})
\end{aligned}
$$

(all but finitely many polyhedra in this sum are equal to $\{0\}$; for $l=k+1$, this is a conventional additivity). Unexpectedly, the assumption of similarity for $A_{0}, \ldots, A_{l}$ can be significantly relaxed in some cases (see Sect. 6.4 for examples), and it would be interesting to know to what extent it can be relaxed in general.

The paper is organized as follows. In Sect. 2, we recall necessary facts and notation, related to convex geometry and Newton polyhedra. In Sect. 3, we study the universal case of our problem, which generalizes results of [14] and [25]. In Sect. 4, we study the special case $l=k$ of our problem (Theorem 4.3), which is a local version of elimination theory in the context of Newton polyhedra [13], and is based on a certain local version of D. Bernstein's formula (Theorem 2.15). In Sect. 5, we apply elimination theory to study the special case $l=0$ (Theorem 5.10). In Sect. 6, we reduce the general case $0 \leqslant l \leqslant k$ to the case $l=0$ by means of a classical technique, known as the Cayley trick, or Lagrange multipliers (Theorem 6.4).

In particular, if the Newton polyhedra of $F_{0}, \ldots, F_{l}$ are not too "thin," then the discriminant set $\Sigma$ is a hypersurface (see Propositions 5.2 for $l=0$ or 6.2 for arbitrary $l$ ), and its Newton polyhedron can be computed by Theorems 5.10 and 6.4. By "thin" we mean Newton polyhedra, such that the collection of polyhedra $A_{0}, \ldots, A_{l}$ (see above) is dual defect. One simple test for non-dual defectiveness is provided by Propositions 3.14 (for $l=0$ ) and 3.24.

We also study the same problem as formulated in the beginning, with another definition of the discriminant: we can define the discriminant set as the minimal set $S$ in $(\mathbb{C} \backslash 0)^{n}$, such that the restriction of the projection $(\mathbb{C} \backslash 0)^{n} \times(\mathbb{C} \backslash 0)^{k} \rightarrow(\mathbb{C} \backslash 0)^{n}$ onto the complete intersection $\left\{F_{0}=\cdots=F_{l}=0\right\}$ is a fiber bundle outside of $S$. This version of the problem is outlined in Sect. 6.5. For example, if the polyhedron $A$ is 
Delzant in the assumptions of the theorem above, then the Newton polyhedron of the equation of $S$ equals

$$
\sum_{\substack{a_{0}>0, \ldots, a_{l}>0 \\ a_{0}+\cdots+a_{l}=k+1}} \Delta_{0}^{a_{0}} \cdots \Delta_{l}^{a_{l}}
$$

(see Corollary 6.18). The counterpart of dual-defectiveness for this problem seems to behave much more simply: see Proposition 3.29 and Conjecture 3.28.

\section{Mixed Volumes, Mixed Fiber Polyhedra, and Euler Obstructions}

In this section, we recall relevant facts and notation from convex geometry: mixed fiber polyhedra [13, 23], Euler obstructions of polyhedra ([20]), relative mixed volume [8, 9], and the corresponding relative version of the KouchnirenkoBernstein formula [1, 19]. Sections 2.4 and 2.6 contain generalizations of the relative Kouchnirenko-Bernstein formula that provide a simple proof for the Matsui-Takeuchi formula for Euler obstructions and for the Gelfand-KapranovZelevinsky decomposition formula; we do not need these generalizations for other purposes. Material from other subsections is used in the proof of our main result.

\subsection{Relative Mixed Volume}

Classical Mixed Volume Recall the notion of the mixed volume of bounded polyhedra. The set $\mathcal{M}$ of all bounded polyhedra in $\mathbb{R}^{m}$ is a semigroup with respect to the Minkowski summation $P+Q=\{p+q \mid p \in P, q \in Q\}$.

Definition 2.1 The mixed volume of polyhedra is the symmetric multilinear function $\mathrm{MV}: \underbrace{\mathcal{M} \times \cdots \times \mathcal{M}}_{m} \rightarrow \mathbb{R}$, such that $\operatorname{MV}(P, \ldots, P)$ equals the volume of $P$ for every $P \in \mathcal{M}$

Lemma 2.2 [19] $\operatorname{MV}\left(\Delta_{1}, \ldots, \Delta_{m}\right)=0$ if and only if $\operatorname{dim} \Delta_{i_{1}}+\cdots+\Delta_{i_{J}}<J$ for some $i_{1}<\cdots<i_{J}$.

This fact is mentioned as obvious in [19], but we prefer to give a proof for the sake of completeness.

Proof $(\Leftarrow)$ follows by an explicit computation if $\Delta_{i_{1}}=\cdots=\Delta_{i_{J}}=C$ is a cube, and all other $\Delta_{i}$ are equal to an $m$-dimensional cube with a face $C$; the general case can be reduced to this one by monotonicity of the mixed volume. In the other direction, consider points $a_{i} \in \Delta_{i}$ and $b_{i} \in \Delta_{i}$ such that the vectors $a_{1}-b_{1}, \ldots, a_{m}-b_{m}$ are in general position in the sense that the dimension of the space generated by $a_{i_{1}}-b_{i_{1}}, \ldots, a_{i_{J}}-b_{i_{J}}$ is the maximal possible one for every subset $\left\{i_{1}, \ldots, i_{J}\right\} \subset\{1, \ldots, m\}$. By monotonicity of the mixed volume, the mixed volume of the segments, connecting $a_{i}$ and $b_{i}$, equals zero, which means that the 
vectors $a_{1}-b_{1}, \ldots, a_{m}-b_{m}$ are linearly dependent. In particular, there exists a minimal subset $\left\{i_{1}, \ldots, i_{J}\right\} \subset\{1, \ldots, m\}$ such that the vectors $a_{i_{1}}-b_{i_{1}}, \ldots, a_{i_{J}}-b_{i_{J}}$ are linearly dependent. They generate a proper subspace $L \subset \mathbb{R}^{m}$, and every $J-1$ of them form a basis of $L$. If there exists $b_{i_{j}}^{\prime} \in \Delta_{i_{j}}$ such that $a_{i_{j}}-b_{i_{j}}^{\prime} \notin L$, then the vectors $a_{i_{1}}-b_{i_{1}}, \ldots, a_{i_{J}}-b_{i_{J}}$ with $a_{i_{j}}-b_{i_{j}}^{\prime}$ instead of $a_{i_{j}}-b_{i_{j}}$ generate a subspace $L^{\prime} \nsupseteq L$, which contradicts the condition of general position. Thus, $\Delta_{i_{1}}, \ldots, \Delta_{i_{J}}$ are contained in a $(J-1)$-dimensional subspace $L$, up to a parallel translation, and $\operatorname{dim} \Delta_{i_{1}}+\cdots+\Delta_{i_{J}}<J$.

Relative Mixed Volume We need the following relative version of the mixed volume. For a convex polyhedral $m$-dimensional cone $\tau \subset\left(\mathbb{R}^{m}\right)^{*}$, denote its dual cone $\{x \in$ $\mathbb{R}^{m} \mid \gamma(x)>0$ for $\left.\gamma \in \tau\right\}$ by $\tau^{\vee}$, and let $\mathcal{M}_{\tau^{\vee}}$ be the semigroup of all (unbounded) polyhedra of the form

$$
\tau^{\vee}+\text { a bounded polyhedron. }
$$

Consider the set $\mathcal{P}_{\tau^{\vee}} \subset \mathcal{M}_{\tau^{\vee}} \times \mathcal{M}_{\tau^{\vee}}$ of all ordered pairs of polyhedra $(P, Q)$, such that the symmetric difference $P \triangle Q$ is bounded. $\mathcal{P}_{\tau} \vee$ is a semigroup with respect to the Minkowski summation of pairs $(P, Q)+(C, D)=(P+C, Q+D)$.

Definition 2.3 $[8,9]$ The volume $V(P, Q)$ of a pair of polyhedra $(P, Q) \in \mathcal{P}_{\Gamma}$ is defined to be the difference $\operatorname{Vol}(P \backslash Q)-\operatorname{Vol}(Q \backslash P)$. The mixed volume of pairs of polyhedra is defined to be the symmetric multilinear function MV : $\underbrace{\mathcal{P}_{\tau^{\vee}} \times \cdots \times \mathcal{P}_{\tau^{\vee}}}_{m} \rightarrow$ $\mathbb{R}$, such that $\operatorname{MV}((P, Q), \ldots,(P, Q))=V(P, Q)$ for every pair $(P, Q) \in \mathcal{P}_{\tau^{\vee}}$.

Existence and uniqueness are proved in [9].

Example 2.4 If $\tau^{\vee}=\{0\}$, then $\mathcal{P}_{\tau^{\vee}}$ is the set of pairs of convex bounded polyhedra, and the mixed volume of pairs $\operatorname{MV}\left(\left(P_{1}, Q_{1}\right), \ldots,\left(P_{m}, Q_{m}\right)\right)$ equals the difference of classical mixed volumes of the collections $P_{1}, \ldots, P_{m}$ and $Q_{1}, \ldots, Q_{m}$.

In general, the mixed volume of pairs can be expressed in terms of the classical mixed volume as follows.

Lemma 2.5 $[9,12]$ Let $\widetilde{P}_{i} \subseteq P_{i}$ and $\widetilde{Q}_{i} \subset Q_{i}$ be bounded polyhedra in $\mathbb{R}^{m}$, such that $\widetilde{P}_{i} \backslash \widetilde{Q}_{i}=P_{i} \backslash Q_{i}$ and $\widetilde{Q}_{i} \backslash \widetilde{P}_{i}=Q_{i} \backslash P_{i}$ for $i=1, \ldots, m$. Then

$$
\operatorname{MV}\left(\left(P_{1}, Q_{1}\right), \ldots,\left(P_{m}, Q_{m}\right)\right)=\operatorname{MV}\left(\widetilde{P}_{1}, \ldots, \widetilde{P}_{m}\right)-\operatorname{MV}\left(\widetilde{Q}_{1}, \ldots, \widetilde{Q}_{m}\right) .
$$

Note that, for any pair $\left(P_{i}, Q_{i}\right)$, we can always find the requested bounded polyhedra $\widetilde{P}_{i}$ and $\widetilde{Q}_{i}$.

The cone $\tau^{\vee}$ plays the role of the unit in the semigroup $\mathcal{P}_{\tau^{\vee}}$ :

Lemma 2.6 [9, 12]

(1) $\operatorname{MV}\left(\left(\tau^{\vee}, Q_{1}\right),\left(P_{2}, Q_{2}\right), \ldots,\left(P_{m}, Q_{m}\right)\right)=\operatorname{MV}\left(\left(\tau^{\vee}, Q_{1}\right),\left(Q_{2}, Q_{2}\right), \ldots,\left(Q_{m}\right.\right.$, $\left.Q_{m}\right)$ ), i.e., the left-hand side does not depend on the choice of $P_{2}, \ldots, P_{m}$.

(2) $\operatorname{MV}\left(\left(\tau^{\vee}, \tau^{\vee}\right),\left(P_{2}, Q_{2}\right), \ldots,\left(P_{m}, Q_{m}\right)\right)=0$. 
Mixed Volume of a Prism Let $e_{1}, \ldots, e_{l}$ be the standard basis of $\mathbb{R}^{l}$, and $e_{0}$ be $0 \in \mathbb{R}^{l}$. For bounded polyhedra $P_{0}, \ldots, P_{l}$ in $\mathbb{R}^{m}$ and a subset $I \subset\{0, \ldots, l\}$, denote the convex hull of the union of the polyhedra $P_{i} \times\left\{e_{i}\right\} \subset \mathbb{R}^{m} \oplus \mathbb{R}^{l}, i \in I$, by $P_{I}$. In what follows, it will be convenient to denote the mixed volume of bounded polyhedra $Q_{1}, \ldots, Q_{m}$ in $\mathbb{R}^{m}$ by the monomial $Q_{1} \cdots Q_{m}$.

\section{Lemma 2.7}

$$
\sum_{I \subset\{0, \ldots, l\}}(-1)^{l+1-|I|}(m+|I|-1) ! \operatorname{Vol}\left(P_{I}\right)=\sum_{\substack{a_{0}>0, \ldots, a_{l}>0 \\ a_{0}+\cdots+a_{l}=m}} m ! P_{0}^{a_{0}} \cdots P_{l}^{a_{l}} .
$$

Proof Pick generic polynomials $g_{0}, \ldots, g_{l}$ whose Newton polyhedra are $P_{0}, \ldots, P_{l}$. Compute the Euler characteristic of the hypersurface $\lambda_{0} g_{0}+\cdots+\lambda_{l} g_{l}=0$ in $\mathbb{C P}_{\lambda_{0}: \cdots: \lambda_{l}}^{l} \times(\mathbb{C} \backslash 0)^{m}$ in the following two ways.

(1) The subdivision of the toric variety $\mathbb{C P}_{\lambda_{0}: \cdots: \lambda_{l}}^{l} \times(\mathbb{C} \backslash 0)^{m}$ into complex tori $T_{I}=\left\{\lambda_{i}=0\right.$ for $\left.i \notin I\right\}$ induces the subdivision of the hypersurface into pieces, whose Euler characteristics equal $(-1)^{l+1-|I|}(m+|I|-1) ! \operatorname{Vol}\left(P_{I}\right)$ by the Kouchnirenko-Khovanskii formula [19]. By additivity, the Euler characteristic of the hypersurface $\lambda_{0} g_{0}+\cdots+\lambda_{l} g_{l}=0$ equals the left-hand side of the desired equality.

(2) Considering the projection of the hypersurface $\lambda_{0} g_{0}+\cdots+\lambda_{l} g_{l}=0$ to $(\mathbb{C} \backslash 0)^{m}$, we note that the fiber of this projection over a point $y \in(\mathbb{C} \backslash 0)^{m}$ equals $\mathbb{C P} l$ or $\mathbb{C P}^{l-1}$ depending on whether $y \in\left\{g_{0}=\cdots=g_{l}=0\right\}$ or not. Thus, integrating the Euler characteristic over fibers of this projection, we conclude that the Euler characteristic of the hypersurface $\lambda_{0} g_{0}+\cdots+\lambda_{l} g_{l}=0$ equals the Euler characteristic of the complete intersection $\left\{g_{0}=\cdots=g_{l}=0\right\}$. Computing the latter one by the Kouchnirenko-Bernstein-Khovanskii formula [19], we get the right-hand side of the desired equality.

\subsection{Mixed Fiber Polyhedra}

Minkowski Integral $\int \Delta[2]$ Denote the projections of the direct sum $\mathbb{R}^{n} \oplus \mathbb{R}^{k}$ onto the summands by $p$ and $q$ respectively. Consider an integer polyhedron $\Delta \subset \mathbb{R}^{n} \oplus \mathbb{R}^{k}$, whose projection $q(\Delta)$ is bounded, and denote the affine span of $q(\Delta)$ by $S$. Choose the volume form $\mathrm{d} x$ on $S$ such that the volume of the image of $S$ under the projection $\mathbb{R}^{k} \rightarrow \mathbb{R}^{k} / \mathbb{Z}^{k}$ equals $(1+\operatorname{dim} S)$ !, and consider the set $I \subset \mathbb{R}^{n} \oplus \mathbb{R}^{k}$ of points of the form $\int_{q(\Delta)} s(x) \mathrm{d} x$, where $s$ runs over all continuous sections of the projection $q: \Delta \rightarrow q(\Delta)$.

Definition 2.8 The Minkowski integral, or the fiber polytope $\int \Delta$ is the closure of $p(I)$.

Mixed Minkowski Integral [23] Let $\tau^{\vee} \subset \mathbb{R}^{n} \subset \mathbb{R}^{n} \oplus \mathbb{R}^{k}$ be a convex polyhedral cone that does not contain a line. The mixed Minkowski integral, or the mixed 
fiber polyhedron, is the symmetric multilinear polyhedral-valued function MP : $\underbrace{\mathcal{M}_{\tau^{\vee}} \times \cdots \times \mathcal{M}_{\tau^{\vee}}}_{k+1} \rightarrow \mathcal{M}_{\tau^{\vee}}$, such that, for every polyhedron $\Delta \in \mathcal{M}_{\tau^{\vee}}$,

$$
\operatorname{MP}(\Delta, \ldots, \Delta)= \begin{cases}\int \Delta & \text { if } \operatorname{dim} q(\Delta)=n, \\ \tau^{\vee} & \text { otherwise. }\end{cases}
$$

See [13, 23], or [11] for existence, uniqueness, and properties of the mixed fiber polyhedron.

Example 2.9 If $\Delta$ is the product of $P \subset \mathbb{R}^{n}$ and $Q \subset \mathbb{R}^{k}$, then $\int \Delta$ equals $((n+$ $1)$ ! Vol $Q) \cdot P$. If $n=1$ and $\tau^{\vee}=\{0\}$, then $\operatorname{MP}\left(\Delta_{0}, \ldots, \Delta_{n}\right)$ is a segment of length $(n+1) ! \operatorname{MV}\left(\Delta_{0}, \ldots, \Delta_{n}\right)$.

We need the following formula for the support function of the mixed fiber polyhedron. Let $\Delta_{0}, \ldots, \Delta_{k}$ be polyhedra in $\mathcal{M}_{\tau^{\vee}}$, and denote the set of positive real numbers by $\mathbb{R}_{+} \subset \mathbb{R}$, and the product $\mathbb{R}_{+} \times q\left(\Delta_{i}\right) \subset \mathbb{R} \oplus \mathbb{R}^{k}$ by $B_{i}$. For every linear function $l: \mathbb{R}^{n} \rightarrow \mathbb{R}$, denote the image of $\Delta_{i}$ under the map $(l$, id $): \mathbb{R}^{n} \oplus \mathbb{R}^{k} \rightarrow \mathbb{R} \oplus \mathbb{R}^{k}$ by $l \Delta_{i}$.

Proposition 2.10 [11] The minimal value of a linear function $l: \mathbb{R}^{n} \rightarrow \mathbb{R}$ on $\operatorname{MP}\left(\Delta_{0}, \ldots, \Delta_{k}\right)$ equals

$$
(k+1) ! \operatorname{MV}\left(\left(l \Delta_{0}, B_{0}\right), \ldots,\left(l \Delta_{k}, B_{k}\right)\right)
$$

if $l \in \tau$ and equals $-\infty$ otherwise.

The proof is given in [11] under an inessential assumption that the polyhedra are bounded.

We also need a slightly more flexible version of the notation above. For an $l$ dimensional vector space $L \subset \mathbb{R}^{k}$, consider the semigroup $\mathcal{M}_{\tau^{\vee}}(L)$ of all polyhedra of the form

$$
Q+\tau^{\vee} \times\{x\} \subset \mathbb{R}^{n} \oplus \mathbb{R}^{k},
$$

where $x$ is a point in $\mathbb{R}^{k}$ and $Q$ is a bounded polyhedron in $\mathbb{R}^{n} \oplus L$.

Definition 2.11 The mixed Minkowski integral, or the mixed fiber polyhedron, is the symmetric multilinear polyhedral-valued function

$$
\mathrm{MP}: \underbrace{\mathcal{M}_{\tau^{\vee}}(L) \times \cdots \times \mathcal{M}_{\tau^{\vee}}(L)}_{l+1} \rightarrow \mathcal{M}_{\tau^{\vee}}(0),
$$

such that, for every polyhedron $\Delta \in \mathcal{M}_{\tau} \vee(L)$,

$$
\operatorname{MP}(\Delta, \ldots, \Delta)= \begin{cases}\int \Delta & \text { if } \operatorname{dim} q(\Delta)=l, \\ \tau^{\vee} & \text { otherwise }\end{cases}
$$


Note that the value $\operatorname{MP}\left(\Delta_{0}, \ldots, \Delta_{l}\right)$ does not depend on the choice of the cone $\tau^{\vee}$ and the space $L$ in the definition above. More precisely, the cone $\tau^{\vee}$ is uniquely defined by the arguments $\Delta_{0}, \ldots, \Delta_{l}$. The space $L$ is also uniquely defined by $\Delta_{0}, \ldots, \Delta_{l}$, provided that the projection $q\left(\Delta_{0}+\cdots+\Delta_{l}\right)$ is $l$-dimensional; otherwise, $\operatorname{MP}\left(\Delta_{0}, \ldots, \Delta_{l}\right)=\tau^{\vee}$ independently of the choice of $L$.

Minkowski Integral of a Prism In what follows, it is convenient to denote the mixed fiber polyhedron $\operatorname{MP}\left(\Delta_{0}, \ldots, \Delta_{l}\right)$ by the monomial $\Delta_{0} \cdots \Delta_{l}$, as we do for the mixed volume (this agrees with Example 2.9). Let $e_{1}, \ldots, e_{l}$ be the standard basis of $\mathbb{R}^{l}$, and $e_{0}$ be $0 \in \mathbb{R}^{l}$. For polyhedra $P_{0}, \ldots, P_{l}$ in $\mathcal{M}_{\tau^{\vee}}$ and a subset $I \subset\{0, \ldots, l\}$, denote the convex hull of the union of the polyhedra $P_{i} \times\left\{e_{i}\right\} \subset \mathbb{R}^{n} \oplus \mathbb{R}^{k} \oplus \mathbb{R}^{l}, i \in I$, by $P_{I}$.

Lemma 2.12 For polyhedra $P_{0}, \ldots, P_{l}$ in $\mathcal{M}_{\tau^{\vee}}$,

(1) $P_{\{0, \ldots, l\}}^{k+l+1}=\sum_{\substack{a_{0} \geqslant 0, \ldots, a_{l} \geqslant 0 \\ a_{0}+\cdots+a_{l}=k+1}} P_{0}^{a_{0}} \cdots P_{l}^{a_{l}}$.

(2) $\sum_{I \subset\{0, \ldots, l\}}(-1)^{l+1-|I|} P_{I}^{k+|I|}=\sum_{\substack{a_{0}>0, \ldots, a_{l}>0 \\ a_{0}+\cdots+a_{l}=k+1}} P_{0}^{a_{0}} \cdots P_{l}^{a_{l}}$.

Proof These two formulas are equivalent by the inclusion-exclusion formula, and we prove the second one. Substituting mixed fiber polyhedra with mixed volumes of pairs by Proposition 2.10, and then with classical mixed volumes by Lemma 2.5, it is enough to prove the same formula for mixed volumes of bounded polyhedra, which is the statement of Lemma 2.7.

\subsection{Kouchnirenko-Bernstein Formula}

The relative version of the classical mixed volume participates in a certain relative version of the classical Kouchnirenko-Bernstein formula. To formulate it, we need some notation related to toric varieties, Newton polyhedra, and intersection numbers.

Toric Varieties For a rational fan $\Sigma$ in $\left(\mathbb{R}^{m}\right)^{*}$, the corresponding toric variety is denoted by $\mathbb{T}^{\Sigma}$. For every codimension 1 orbit $T$ of $\mathbb{T}^{\Sigma}$, the primitive generator of the corresponding 1-dimensional cone of $\Sigma$ is denoted by $\gamma(T)$. We assume that the union of cones of $\Sigma$ is a closed convex cone $\tau$, and denote its dual by $\tau^{\vee} \subset \mathbb{R}^{m}$.

If $I$ is a very ample line bundle on $\mathbb{T}^{\Sigma}$, and a meromorphic section $s$ of the bundle $I$ has no zeros and no poles in the maximal torus of $\mathbb{T}^{\Sigma}$, then there exists a unique polyhedron $\Delta \in \mathcal{M}_{\tau^{\vee}}$, such that the multiplicity of every codimension 1 orbit $T$ of $\mathbb{T}^{\Sigma}$ in the divisor of poles and zeros of the section $s$ equals the maximal value of the linear function $-\gamma(T): \mathbb{R}^{m} \rightarrow \mathbb{R}$ on the polyhedron $\Delta$. Since the pair $(I, s)$ is uniquely determined by this polyhedron $\Delta$, we denote the line bundle $I$ by $I_{\Delta}$ and the section $s$ by $s_{\Delta}$.

Newton Polyhedra The union of all precompact orbits of the toric variety $\mathbb{T}^{\Sigma}$ (i.e., the orbits corresponding to the cones of $\Sigma$ in the interior of $\tau$ ) is denoted by $\mathbb{T}_{\text {comp }}^{\Sigma}$ and is called the compact part of $\mathbb{T}^{\Sigma}$ (it is indeed a compact set). 
If $f$ is an arbitrary germ of a holomorphic section of $I_{\Delta}$ near the compact set $\mathbb{T}_{\text {comp }}^{\Sigma}$, then the function $f / s_{\Delta}$ can be represented as a power series $\sum_{a \in \Delta} c_{a} x^{a}$ for $x$ in the maximal torus $(\mathbb{C} \backslash 0)^{m}$ of the toric variety $\mathbb{T}^{\Sigma}$. The convex hull of the set $\left\{a \mid c_{a} \neq 0\right\}+\tau^{\vee}$ is an integer polyhedron in $\mathcal{M}_{\tau^{\vee}}$. It is called the Newton polyhedron of $f$ and is denoted by $\Delta_{f}$. For any bounded $\Gamma \subset \mathbb{R}^{m}$, the polynomial $\sum_{a \in \Gamma} c_{a} x^{a}$ on $(\mathbb{C} \backslash 0)^{m}$ is denoted by $f^{\Gamma}$. If $a$ is contained in a bounded face of the Newton polyhedron $\Delta_{f}$, then the coefficient $c_{a}$ is called a leading coefficient of $f$. Every section has a finite number of leading coefficients.

Intersection Numbers Let $f_{1}, \ldots, f_{k}$ be continuous sections of complex line bundles $I_{1}, \ldots, I_{k}$ on a $k$-dimensional complex algebraic variety $V$, such that the set $\left\{f_{1}=\cdots=f_{k}=0\right\}$ is compact. Consider the Chern class $c_{i} \in H^{2}\left(V,\left\{f_{i} \neq 0\right\} ; \mathbb{Z}\right)$ of the bundle $I_{i}$, localized near the zero locus of its section $f_{i}$. Then the intersection number of the divisors of the sections $f_{1}, \ldots, f_{k}$ is defined as $c_{1} \smile \cdots \smile c_{k} \in$ $H^{2 k}\left(V, \bigcup_{i}\left\{f_{i} \neq 0\right\} ; \mathbb{Z}\right)=\mathbb{Z}$ and is denoted by $m\left(f_{1} \cdots f_{k} \cdot V\right)$.

In other words, if we consider smooth (nonholomorphic) perturbations $\tilde{f}_{1}, \ldots, \tilde{f}_{k}$ of the sections $f_{1}, \ldots, f_{k}$, such that the system $\tilde{f}_{1}=\cdots=\tilde{f}_{k}=0$ has finitely many regular solutions near the set $\left\{f_{1}=\cdots=f_{k}=0\right\}$, then each of the solutions can be assigned a weight \pm 1 , depending on the orientation of the base $d \tilde{f}_{1}, \ldots, d \tilde{f}_{k}$ at this point. The intersection number $m\left(f_{1} \cdots f_{k} \cdot V\right)$ by definition equals the sum of these weights.

Relative Kouchnirenko-Bernstein Formula Let $\Delta_{1}, \ldots, \Delta_{m}$ be integer polyhedra in $\mathcal{M}_{\tau} \vee$, and let $\widetilde{\Delta}_{1}, \ldots, \widetilde{\Delta}_{m}$ be the Newton polyhedra of germs of sections $f_{1}, \ldots, f_{m}$ of the line bundles $I_{\Delta_{1}}, \ldots, I_{\Delta_{m}}$ on the toric variety $\mathbb{T}^{\Sigma}$. We compute the intersection number of the divisors of the sections $f_{1}, \ldots, f_{m}$ in terms of the polyhedra $\Delta_{1}, \ldots, \Delta_{m}$ and $\widetilde{\Delta}_{1}, \ldots, \widetilde{\Delta}_{m}$, provided that the leading coefficients of $f_{1}, \ldots, f_{m}$ are in general position.

Definition 2.13 For every face $\Gamma$ of the sum of polyhedra $\Delta_{1}, \ldots, \Delta_{m}$ in $\mathbb{R}^{p}$, the maximal collection of faces $\Gamma_{1} \subset \Delta_{1}, \ldots, \Gamma_{m} \subset \Delta_{m}$, such that $\Gamma_{1}+\cdots+\Gamma_{m}=\Gamma$, is said to be compatible.

For bounded faces, the word "maximal" can be omitted in this definition.

Definition 2.14 The leading coefficients of the sections $f_{1}, \ldots, f_{m}$ are said to be in general position if, for every collection of bounded compatible faces $\widetilde{\Gamma}_{1}, \ldots, \widetilde{\Gamma}_{m}$ of the polyhedra $\widetilde{\Delta}_{1}, \ldots, \widetilde{\Delta}_{m}$, the system of polynomial equations $f_{1}^{\widetilde{\Gamma}_{1}}=\cdots=f_{m} \widetilde{\Gamma}_{m}=0$ has no roots in the maximal torus $(\mathbb{C} \backslash 0)^{m}$.

Theorem 2.15 (Relative Kouchnirenko-Bernstein formula, [8, 9]) Let $\Delta_{1}, \ldots, \Delta_{m}$ be integer polyhedra in $\mathcal{M}_{\tau} \vee$, and let $\widetilde{\Delta}_{1}, \ldots, \widetilde{\Delta}_{m}$ be the Newton polyhedra of sections $f_{1}, \ldots, f_{m}$ of the line bundles $I_{\Delta_{1}}, \ldots, I_{\Delta_{m}}$, such that the difference $\Delta_{i} \backslash \widetilde{\Delta}_{i}$ is bounded for every $i$. Then

(1) The intersection number $m\left(f_{1} \cdots f_{m} \cdot \mathbb{T}^{\tau}\right)$ is greater than or equal to the mixed volume $m ! \operatorname{MV}\left(\left(\Delta_{1}, \widetilde{\Delta}_{1}\right), \ldots,\left(\Delta_{m}, \widetilde{\Delta}_{m}\right)\right)$. 
(2) This inequality turns into an equality if and only if leading coefficients of the sections $f_{1}, \ldots, f_{m}$ are in general position in the sense of Definition 2.14.

\subsection{Kouchnirenko-Bernstein-Khovanskii Formula}

In the assumptions of Theorem 2.15, suppose that the first line bundle $\mathcal{I}_{\Delta_{1}}$ is trivial, i.e., $\Delta_{1}=\tau^{\vee}$. The relative version of the Kouchnirenko-Bernstein-Khovanskii formula computes the Euler characteristic of the Milnor fiber of the function $f_{1}$ on the complete intersection $f_{2}=\cdots=f_{k}=0$ for $k \leqslant m$, in terms of the Newton polyhedra of the sections $f_{1}, \ldots, f_{k}$. At the end of this subsection, we also explain how to drop the assumption on the triviality of $\mathcal{I}_{\Delta_{1}}$.

To define the Milnor fiber of $s_{1}$, it is convenient to fix a family of neighborhoods for the compact part of the toric variety $\mathbb{T}^{\Sigma}$. For instance, choose an integer point $a_{i}$ on every infinite edge of $\Delta_{1}$, and let $B_{\varepsilon}$ be the set of all $x \in(\mathbb{C} \backslash 0)^{m}$ such that $\sum_{i}\left|x^{a_{i}}\right| \leqslant \varepsilon$. Then its closure in the toric variety $\mathbb{T}^{\Sigma}$ is a neighborhood of the compact part $\mathbb{T}_{\text {comp }}^{\Sigma}$.

Definition 2.16 The Milnor fiber of the function $f_{1}$ on the complete intersection $f_{2}=\cdots=f_{k}=0$ is the manifold $\left\{f_{1}-\delta=f_{2}=\cdots=f_{k}=0\right\} \cap B_{\varepsilon}$ for $|\delta| \ll \varepsilon \ll 1$.

Definition 2.17 The leading coefficients of $f_{1}, \ldots, f_{k}$, are said to be in general position if, for every collection of bounded compatible faces $\widetilde{\Gamma}_{1}, \ldots, \widetilde{\Gamma}_{k}$ of the polyhedra $\widetilde{\Delta}_{1}, \ldots, \widetilde{\Delta}_{k}$, the systems of polynomial equations $f_{1}^{\widetilde{\Gamma}_{1}}=\cdots=f_{k}^{\widetilde{\Gamma}_{k}}=0$ and $f_{2}^{\widetilde{\Gamma}_{2}}=\cdots=f_{k}^{\widetilde{\Gamma}_{k}}=0$ define regular varieties in the maximal torus $(\mathbb{C} \backslash 0)^{m}$.

We denote the mixed volume of pairs of polyhedra $\left(P_{1}, Q_{1}\right), \ldots,\left(P_{m}, Q_{m}\right)$ in $\mathbb{R}^{m}$ by the monomial $\left(P_{1}, Q_{1}\right) \cdots\left(P_{m}, Q_{m}\right)$.

Theorem 2.18 In the above assumptions, the Euler characteristic of the Milnor fiber of $f_{1}$ on the complete intersection $\left\{f_{2}=\cdots=f_{k}=0\right\}$ equals

$$
(-1)^{m-k} m ! \sum_{\substack{a_{1}>0, \ldots, a_{k}>0 \\ a_{1}+\cdots+a_{k}=m}}\left(\Delta_{1}, \widetilde{\Delta}_{1}\right)^{a_{1}} \cdots\left(\Delta_{k}, \widetilde{\Delta}_{k}\right)^{a_{k}},
$$

provided that the leading coefficients of $f_{1}, \ldots, f_{k}$ are in general position in the sense of Definition 2.17.

This is proved in [24] for a regular affine toric variety with arbitrary $k$ (based on the idea of [29]), and in [21] for an arbitrary affine toric variety (in more up-todate language). Both reasonings can be easily applied to an arbitrary (not necessarily affine) toric variety, and also provide a formula for the $\zeta$-function of monodromy of the function $f_{1}$. However, since we restrict our consideration to the Milnor number in this paper, we prefer to give a much simpler proof by reduction to the global Kouchnirenko-Bernstein-Khovanskii formula. 
Proof If the leading coefficients are in general position, then the topology of the Milnor fiber only depends on the Newton polyhedra of $f_{1}, \ldots, f_{k}$, and we can assume without loss of generality that $f_{i}=s_{\Delta_{i}} \cdot \tilde{f}_{i}$, where $\tilde{f}_{1}, \ldots, \tilde{f}_{k}$ are Laurent polynomials on $(\mathbb{C} \backslash 0)^{m}$ and satisfy the condition of general position of [19]. Denote the Newton polyhedra of the polynomials $\tilde{f}_{1}, \ldots, \tilde{f}_{k}$ and $\tilde{f}_{1}-\delta$ with $\delta \neq 0$ by $D_{1}, \ldots, D_{k}$ and $\widetilde{D}_{1}$.

By the global Kouchnirenko-Bernstein-Khovanskii formula [19], the Euler characteristics of $\left\{\tilde{f}_{1}=\cdots=\tilde{f}_{k}=0\right\}$ and $\left\{\tilde{f}_{1}-\delta=\tilde{f}_{2}=\cdots=\tilde{f}_{k}=0\right\}$ equal $(-1)^{m-k} m ! \sum_{\substack{a_{1}>0, \ldots, a_{k}>0 \\ a_{1}+\cdots+a_{k}=m}}^{\mathrm{red}} D_{1}^{a_{1}} \cdots \cdot D_{k}^{a_{k}}$ and $(-1)^{m-k} m ! \sum_{\substack{a_{1}>0, \ldots, a_{k}>0 \\ a_{1}+\cdots+a_{k}=m}}^{\mathrm{red}} \widetilde{D}_{1}^{a_{1}} \cdot D_{2}^{a_{2}}$ $\cdots D_{k}^{a_{k}}$ respectively.

Since the boundary of $B_{\varepsilon}$ subdivides the set $\left\{\tilde{f}_{1}-\delta=\tilde{f}_{2}=\cdots=\tilde{f}_{k}=0\right\}$ into two parts, homeomorphic to the set $\left\{\tilde{f}_{1}=\cdots=\tilde{f}_{k}=0\right\}$ and the Milnor fiber of $f_{1}$ on $\left\{f_{2}=\cdots=f_{n}=0\right\}$, the Euler characteristic of the latter equals

$$
(-1)^{m-k} m ! \sum_{\substack{a_{1}>0, \ldots, a_{k}>0 \\ a_{1}+\cdots+a_{k}=m}} \widetilde{D}_{1}^{a_{1}} \cdot D_{2}^{a_{2}} \cdots D_{k}^{a_{k}}-(-1)^{m-k} m ! \sum_{\substack{a_{1}>0, \ldots, a_{k}>0 \\ a_{1}+\cdots+a_{k}=m}} D_{1}^{a_{1}} \cdots D_{k}^{a_{k}}
$$

by the additivity of the Euler characteristic. This difference is equal to

$$
(-1)^{m-k} m ! \sum_{\substack{a_{1}>0, \ldots, a_{k}>0 \\ a_{1}+\cdots+a_{k}=m}}\left(\Delta_{1}, \widetilde{\Delta}_{1}\right)^{a_{1}} \cdots\left(\Delta_{k}, \widetilde{\Delta}_{k}\right)^{a_{k}}
$$

by Lemmas 2.5 and 2.6.

Summing up the Euler characteristics of the Milnor fibers of $\left.f_{1}\right|_{\left\{f_{2}=\cdots=f_{k}=0\right\}}$ over all noncompact toric subvarieties in $\mathbb{T}^{\Sigma}$, we have the following formula for the Euler characteristic of the closure of the Milnor fiber.

The fact that $\mathcal{I}_{\Delta_{i}}$ is a line bundle on the toric variety $\mathbb{T}^{\Sigma}$ implies that, for every cone $\sigma \in \Sigma$, all interior points of $\sigma$, being considered as linear functions on the polyhedron $\Delta_{i}$, attain their minimum on the same face of $\Delta_{i}$. Denote this face by $A_{i}$, the codimension of the cone $\sigma$ by $q$, and pick a vector $a_{i} \in A_{i}$. Then the shifted pairs $\mathcal{A}_{i}=\left(A_{i}-a_{i},\left(A_{i} \cap \widetilde{\Delta}_{i}\right)-a_{i}\right)$ are contained in the same rational $q$-dimensional subspace of $\mathbb{R}^{m}$, and their $q$-dimensional mixed volumes are well defined. Denote the number $(-1)^{q-k} q ! \sum_{\substack{a_{1}>0, \ldots, a_{k}>0 \\ a_{1}+\cdots+a_{k}=q}}^{\mathrm{red}} \mathcal{A}_{1}^{a_{1}} \cdots \mathcal{A}_{k}^{a_{k}}$ by $\chi_{\sigma}$.

Corollary 2.19 In the above assumptions, the Euler characteristic of the closure of the Milnor fiber of $f_{1}$ on the complete intersection $\left\{f_{2}=\cdots=f_{n}=0\right\}$ equals $\sum_{\sigma \in \Sigma} \chi_{\sigma}$, provided that the leading coefficients of $s_{1}, \ldots, s_{k}$ are in general position.

We also formulate a more general version of this theorem, with no assumptions on the triviality of the first line bundle (we do not need this generalization in what follows; since the proof is similar to that of Corollary 2.19, we omit it). Let $f_{1}, \ldots, f_{k}$ be germs of holomorphic sections of arbitrary line bundles $\mathcal{I}_{\Delta_{1}}, \ldots, \mathcal{I}_{\Delta_{k}}$ near the compact part of a toric variety $\mathbb{T}^{\Sigma}$, and pick holomorphic sections $t_{1}, \ldots, t_{k}$ of these bundles in the closure of the set $B_{\varepsilon}$ for a small $\varepsilon$. Varieties $B_{\varepsilon} \cap\left\{f_{1}-t_{1}=\cdots=f_{k}-\right.$ 
$\left.t_{k}=0\right\}$ are diffeomorphic to each other for almost all collections of small sections $\left(t_{1}, \ldots, t_{k}\right)$. Such a variety is called the Milnor fiber of the complete intersection $\left\{f_{1}=\cdots=f_{k}=0\right\}$.

Theorem 2.20 In the above assumptions, the Euler characteristic of the Milnor fiber of the complete intersection $\left\{f_{1}=\cdots=f_{k}=0\right\}$ equals

$$
(-1)^{m-k} m ! \sum_{\substack{a_{1}>0, \ldots, a_{k}>0 \\ a_{1}+\cdots+a_{k}=m}}\left(\Delta_{1}, \widetilde{\Delta}_{1}\right)^{a_{1}} \cdots\left(\Delta_{k}, \widetilde{\Delta}_{k}\right)^{a_{k}},
$$

provided that the leading coefficients of $f_{1}, \ldots, f_{k}$ are in general position.

\subsection{Euler Obstructions of Polyhedra}

Euler Obstructions of Varieties Let $\bigsqcup_{\alpha \in \Lambda} U_{\alpha}$ be a Whitney stratification of a complex algebraic variety $U$. Pick a point $x_{0}$ in a stratum $U_{\alpha^{\prime}}$ and consider a germ of an analytic function $f:\left(U, U_{\alpha^{\prime}}\right) \rightarrow(\mathbb{C}, 0)$ at this point. The Euler characteristic of the set $\left\{x \in U_{\alpha}|f(x)=\delta| x-,x_{0} \mid \leqslant \varepsilon\right\}$ takes the same value for almost all germs $f$ and all positive numbers $\delta \ll \varepsilon \ll 1$. This value does not depend on the choice of $x_{0} \in U_{\alpha^{\prime}}$, and we denote the negative of this value by $\mu^{\alpha^{\prime}, \alpha}$ (note that it equals 0 unless $U_{\alpha}$ is adjacent to $U_{\alpha^{\prime}}$ ). We also set $\mu^{\alpha, \alpha}=1$ for every $\alpha \in \Lambda$ and denote the $|\Lambda| \times|\Lambda|$ matrix with entries $\mu^{\alpha^{\prime}, \alpha}$ by $M$.

Definition 2.21 The $\left(\alpha^{\prime}, \alpha\right)$-entry of the inverse matrix $M^{-1}$ is denoted by $\epsilon^{\alpha^{\prime}, \alpha}$ and is called the Euler obstruction of the closure of the stratum $U_{\alpha}$ at a point of the stratum $U_{\alpha^{\prime}}$.

Remark Adjacency of strata induces a partial order structure on the set $\Lambda$. If we consider the collections $\mu^{\alpha^{\prime}, \alpha}$ and $\epsilon^{\alpha^{\prime}, \alpha}$ as functions on the poset $\Lambda \times \Lambda$, these functions are called Möbius inverse (see, e.g., [16] for details).

Example 2.22 Let $(U, 0)$ be an $m$-dimensional isolated toric singularity (it consists of two strata $U \backslash\{0\}$ and $\{0\}$ ). Denote the $m$-dimensional cone of its fan by $\tau$, the convex hull of $\tau^{\vee} \cap \mathbb{Z}^{n} \backslash\{0\}$ by $H$, and the volume of $\tau^{\vee} \backslash H$ by $V$. Then, by Corollary 2.19, the above matrix $M$ equals $\left(\begin{array}{cc}1 & m ! V-2 \\ 0 & 1\end{array}\right)$ for even $m$ and $\left(\begin{array}{cc}1 & -m ! V \\ 0 & 1\end{array}\right)$ for odd $m$. Thus, the Euler obstruction of $U$ equals $2-m ! V$ for even $m$ and $m ! V$ for odd $m$.

\section{Combinatorial Coefficients $c^{A^{\prime}, A}$ and $e^{A^{\prime}, A}$}

Definition 2.23 A subset $A^{\prime} \subset A \subset \mathbb{Z}^{k}$ is called a face of $A$ if it can be represented as the intersection of $A$ with a face of the convex hull of $A$.

For a face $A^{\prime}$ of a finite set $A \subset \mathbb{R}^{k}$, let $M^{\prime}$ and $M \subset \mathbb{R}^{k}$ be the vector spaces, parallel to the affine spans of $A^{\prime}$ and $A$ respectively. We denote the projection $\mathbb{R}^{k} \rightarrow \mathbb{R}^{k} / M^{\prime}$ by $s$, and choose the volume form $\eta$ on $M / M^{\prime}$ such that the volume of $M /\left(M^{\prime}+\mathbb{Z}^{k}\right)$ equals $\left(\operatorname{dim} M / M^{\prime}\right)$ !. Then the $\eta$-volume of the difference of the 
convex hulls of $s(A)$ and $s\left(A \backslash A^{\prime}\right)$ is denoted by $c^{A^{\prime}, A} \in \mathbb{Z}$. Set $c^{A, A}$ to 1 and $c^{A^{\prime}, A}$ to 0 if $A^{\prime}$ is not a face of $A$.

Example 2.24 The above difference is shown by hatching below for the following two examples:

(1) $A_{1}=\{(0,0,0),(0,0,1),(2,1,0),(1,2,1)\}$ and $A_{1}^{\prime}$ is its vertical edge.

(2) $A_{2}$ is the set of integer lattice points in the convex hull of $2 \cdot A_{1}$, and $A_{2}^{\prime}$ is its vertical edge.

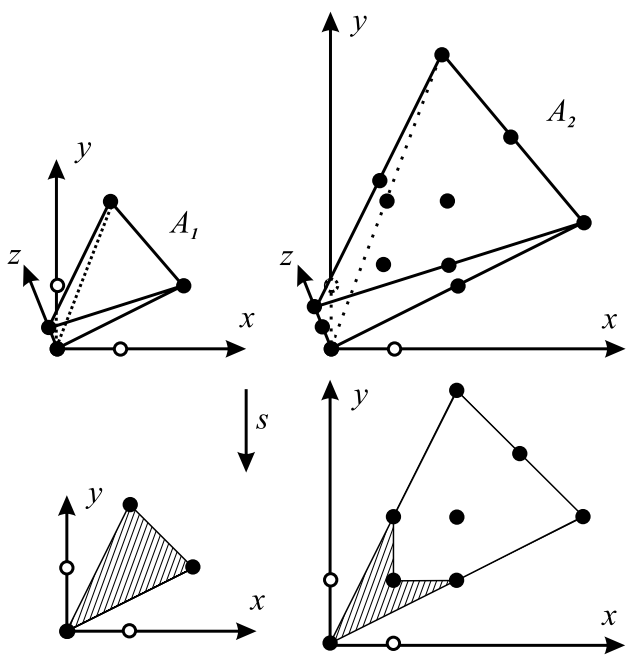

Consider the square matrix $C$ with entries $c^{A^{\prime \prime}, A^{\prime}}$, where $A^{\prime \prime}$ and $A^{\prime}$ run over the set of all faces of $A$, and define $e^{A^{\prime \prime}, A^{\prime}}$ as the $\left(A^{\prime \prime}, A^{\prime}\right)$-entry of the inverse of $C$. Note that $C$ is upper triangular with 1's on the diagonal, if we order faces of $A$ by their dimension; in particular, the determinant of $C$ equals 1 , and its inverse is integer (although positivity of the entries of $C$ does not imply positivity of the entries of its inverse).

Definition 2.25 The number $e^{A^{\prime}, A}$ is called the Euler obstruction of the set $A$ at its face $A^{\prime}$.

For instance, restricting our attention to the four faces of the set $A_{2}$ adjacent to the vertical edge $A_{2}^{\prime}$ in the previous example (including $A_{2}^{\prime}$ itself), we obtain the Euler obstruction $e^{A_{2}, A_{2}^{\prime}}$ as the top right element in the matrix $C^{-1}$ :

$$
C=\left(\begin{array}{cccc}
1 & 1 & 1 & 2 \\
& 1 & 0 & 1 \\
& & 1 & 1 \\
& & & 1
\end{array}\right), \quad C^{-1}=\left(\begin{array}{cccc}
1 & -1 & -1 & 0 \\
& 1 & 0 & -1 \\
& & 1 & -1 \\
& & & 1
\end{array}\right)
$$


For an integer polyhedron $P \subset \mathbb{R}^{m}$ and its face $Q$, we call the numbers $c^{P \cap \mathbb{Z}^{m}, Q \cap \mathbb{Z}^{m}}$ and $e^{P \cap \mathbb{Z}^{m}, Q \cap \mathbb{Z}^{m}}$ the Milnor number and the Euler obstruction of the polyhedron $P$ at its face $Q$, and denote them by $c^{Q, P}$ and $e^{Q, P}$.

Example 2.26 For an integer polygon $P$ at its vertex $Q$, denoting the convex hull of $\mathbb{Z}^{2} \cap P \backslash\{Q\}$ by $P_{Q}$, we have

$$
c^{Q, P}=2 \cdot \text { area of } P \backslash P_{Q}, \quad e^{Q, P}=2-2 \cdot \text { area of } P \backslash P_{Q} .
$$

In particular, if $c^{Q, P}=e^{Q, P}=1$, then the adjacent edges of the vertex $Q$ can be brought to the lines

$$
y=0 \text { and } \quad x=0
$$

by a suitable affine change of coordinates in $\mathbb{R}^{2}$ that preserves the integer lattice. If ${ }_{c} Q, P=2$ (and $e^{Q, P}=0$ ), then the adjacent edges of the vertex $Q$ can be brought to the lines of the form

$$
y=0 \quad \text { and } \quad y=\left(1+\frac{1}{n}\right) x, \quad \text { where } n \in \mathbb{N} .
$$

More generally, if $c^{Q, P}=s$, then the adjacent edges of the vertex $Q$ can be brought to the lines of the form

$$
y=0 \quad \text { and } \quad y=\left(p_{0}+\frac{1}{p_{1}+\cdots \frac{1}{p_{2 k}}}\right) x, \quad \text { where } p_{i} \in \mathbb{N} \quad \text { and } \quad \sum_{i} p_{2 i}=s
$$

(the number in the brackets is the continued fraction of the sequence $p_{0}, \ldots, p_{2 k}$ ). It would be interesting to extend this to classification of $r$-dimensional rational convex polyhedral cones $P$ such that $c^{0, P}=s$ or $e^{0, P}=s$ for small $r$ and $|s|$; this problem is related to problems of classification of multidimensional continued fractions and sails (see, e.g., [17]).

Geometric Meaning of $c^{A^{\prime}, A}$ and $e^{A^{\prime}, A}$ For a set $A=\left\{a_{1}, \ldots, a_{N}\right\} \subset \mathbb{Z}^{k}$, such that the differences $a_{i}-a_{j}$ generate the lattice $\mathbb{Z}^{k}$, the closure of the image of the torus $(\mathbb{C} \backslash 0)^{k}$ under the inclusion $j:(\mathbb{C} \backslash 0)^{k} \rightarrow \mathbb{C P}^{A}, j(t)=\left(t^{a_{1}}, \ldots, t^{a_{N}}\right)$, is a toric variety, whose orbits are in one-to-one correspondence with faces of $A$. Its subdivision into the orbits is a Whitney stratification $\bigsqcup U_{A^{\prime}}$, where $A^{\prime}$ runs over all faces of $A$. This stratification gives rise to the numbers $\mu^{A^{\prime \prime}}, A^{\prime}$ and $\epsilon^{A^{\prime \prime}, A^{\prime}}$ for every pair of faces $A^{\prime}$ and $A^{\prime \prime}$, as defined in the beginning of this subsection.

Since, by Theorem 2.18, we have $\mu^{A^{\prime \prime}}, A^{\prime}=(-1)^{\operatorname{dim} A^{\prime \prime}-\operatorname{dim} A^{\prime}} c^{A^{\prime \prime}, A^{\prime}}$, then $\epsilon^{A^{\prime \prime}, A^{\prime}}=$ $(-1)^{\operatorname{dim} A^{\prime \prime}-\operatorname{dim} A^{\prime}} e^{A^{\prime \prime}}, A^{\prime}$, which proves the following.

Proposition 2.27 [20] The Euler obstruction of the set $A \subset \mathbb{Z}^{k}$ at its face $A^{\prime}$ equals $(-1)^{\operatorname{dim} A^{\prime}-\operatorname{dim} A}$ times the Euler obstruction of the toric variety, corresponding to $A$, at a point of its orbit, corresponding to $A^{\prime}$. 


\subsection{Multiplicities of Nondegenerate Complete Intersections}

Here we formulate a corollary of the relative Kouchnirenko-Bernstein formula that leads to a simple proof of the Gelfand-Kapranov-Zelevinsky decomposition formula (we do not need it for other purposes).

Varieties with Multiplicities Let $f_{1}, \ldots, f_{l}$ be germs of holomorphic sections of complex line bundles $I_{1}, \ldots, I_{l}$ on a germ of a $k$-dimensional complex algebraic variety $(V, x)$, such that the set $\left\{f_{1}=\cdots=f_{l}=0\right\}$ is smooth and $(k-l)$-dimensional. Then we can choose germs of holomorphic functions $f_{l+1}, \ldots, f_{k}$ on $(V, x)$, such that the differentials of their restrictions onto $\left\{f_{1}=\cdots=f_{l}=0\right\}$ are linearly independent. The local topological degree of the map $\left(f_{1}, \ldots, f_{k}\right):(V, x) \rightarrow\left(\mathbb{C}^{k}, 0\right)$ does not depend on the choice of the germs $f_{l+1}, \ldots, f_{k}$ and is called the multiplicity of the (local) complete intersection $f_{1}=\cdots=f_{l}=0$ at its point $x$.

If $S_{i}, i=1, \ldots, I$, are the irreducible components of a complete intersection $\left\{f_{1}=\right.$ $\left.\cdots=f_{l}=0\right\}$, and $a_{i}$ is the multiplicity of this complete intersection at a smooth point of $S_{i}$, then we denote the cycle $\sum_{i=0}^{I} a_{i} S_{i}$ (i.e., a formal sum of irreducible varieties) by $\left[f_{1}=\cdots=f_{l}=0\right]$.

If $S=\sum a_{i} S_{i}$ is a cycle in $V$ (every $S_{i}$ is an irreducible variety in $V$, and every $a_{i}$ is a positive number), and $f: V \rightarrow W$ is a proper map, then we define the image $f_{*}(S)$ as follows. For every component $S_{i}$, denote the topological degree of the map $f: S_{i} \rightarrow f\left(S_{i}\right)$ by $d_{i}$, provided that $\operatorname{dim} f\left(S_{i}\right)=\operatorname{dim} S_{i}$ (otherwise, set $d_{i}=0$ by definition). Then the image $f_{*}(S)$ is defined to be the sum $\sum a_{i} d_{i} f\left(S_{i}\right)$.

Multiplicities of Nondegenerate Complete Intersections Let $\Delta \subset \mathbb{R}^{m}$ be an $m$ dimensional integer polyhedron, let $\Sigma$ be its dual fan, and let $\widetilde{\Delta}$ be the Newton polyhedron of holomorphic sections $f_{1}, \ldots, f_{l}$ of the line bundle $I_{\Delta}$ on the toric variety $\mathbb{T}^{\Sigma}$. Denote the orbit of $\mathbb{T}^{\Sigma}$, corresponding to a codimension $l$ face $\Gamma$ of $\Delta$, by $\mathbb{T}^{\Gamma}$. Then the multiplicity of the complete intersection $f_{1}=\cdots=f_{l}=0$ at a generic point of the orbit $\mathbb{T}^{\Sigma}$ can be computed as follows.

Let $p: \mathbb{R}^{m} \rightarrow \mathbb{R}^{l}$ be a projection such that $p\left(\mathbb{Z}^{m}\right)=\mathbb{Z}^{l}$ and $p(\Gamma)$ is a point.

Lemma 2.28 If $p(\widetilde{\Delta})$ touches all faces of $p(\Delta)$ except the vertex $p(\Gamma)$, and the leading coefficients of $f_{1}, \ldots, f_{l}$ are in general position, then the closure of the orbit $\mathbb{T}^{\Gamma}$ is a component of multiplicity $l ! \operatorname{Vol}(p(\Delta) \backslash p(\widetilde{\Delta}))$ in the complete intersection $f_{1}=\cdots=f_{l}=0$.

The assumption that the Newton polyhedra of the sections $f_{1}, \ldots, f_{l}$ coincide is obviously redundant; it is introduced to simplify the notation.

Proof Let $\mathbb{T}_{\Gamma} \subset(\mathbb{C} \backslash 0)^{m}$ be the $l$-dimensional subtorus that acts trivially on the orbit $\mathbb{T}^{\Gamma}$. For every point $x \subset \mathbb{T}^{\Gamma}$, consider the $\mathbb{T}_{\Gamma}$-invariant $l$-dimensional closed toric subvariety $H_{x} \subset \mathbb{T}^{\Sigma}$ that intersects the orbit $\mathbb{T}^{\Gamma}$ at the point $x$.

We should prove that, for a generic point $x$, the intersection number $\mathcal{J}$ of the variety $H_{x}$ and the complete intersection $f_{1}=\cdots=f_{l}=0$ at $x$ is well defined and equals $l ! \operatorname{Vol}(p(\Delta) \backslash p(\widetilde{\Delta}))$. To do so, we denote the restrictions of the line bundle $I_{\Delta}$ 
and its sections $f_{1}, \ldots, f_{l}$ to $H_{x}$ by $I^{\prime}$ and $f_{1}^{\prime}, \ldots, f_{l}^{\prime}$ respectively and note that $I^{\prime}=$ $I_{p(\Delta)}$ and the Newton polyhedra of $f_{1}^{\prime}, \ldots, f_{l}^{\prime}$ are equal to $p(\widetilde{\Delta})$. On one hand, the intersection number of the divisors of $f_{1}^{\prime}, \ldots, f_{l}^{\prime}$ is equal to the desired intersection number $\mathcal{J}$, while on the other hand it is well defined and equals $l ! \operatorname{Vol}(p(\Delta) \backslash p(\widetilde{\Delta}))$ by Theorem 2.15 for the sections $f_{1}^{\prime}, \ldots, f_{l}^{\prime}$.

\section{A-discriminants}

In this section, we discuss the universal case of our problem. For a collection of finite sets $A_{0}, \ldots, A_{k}$ in $\mathbb{Z}^{k}$, we recall the definition of the $\left(A_{0}, \ldots, A_{k}\right)$-resultant (Sect. 3.1) and the $A_{0}$-discriminant (Sect. 3.2). More generally, for every $l \leqslant k$, we introduce what is called the $\left(A_{0}, \ldots, A_{l}\right)$-discriminant (Sect. 3.4), and express it in terms of $A$-discriminants by means of the Cayley trick (Sect. 3.5).

The collection $A_{0}, \ldots, A_{l}$ is called dual defective if the $\left(A_{0}, \ldots, A_{l}\right)$-discriminant set is not a hypersurface, and we give a number of examples of sufficient conditions for non-dual defectiveness of a collection (Sect. 3.3 for $l=0$ and Proposition 3.24 for arbitrary $l)$. We also consider an alternative version of the $\left(A_{0}, \ldots, A_{l}\right)$-discriminant set (the bifurcation set, see Definition 3.26), which is presumably always a hypersurface (see Proposition 3.29 and the preceding conjecture). The technical proof of Propositions 3.24 and 3.29 and the alternative proof of the Gelfand-KapranovZelevinsky decomposition formula are postponed until the end of this section.

\subsection{Resultants and $A$-determinants ([25] and [14])}

Resultant For a finite set $A \subset \mathbb{Z}^{k}$, denote the set of all Laurent polynomials of the form $\sum_{a \in A} c_{a} x^{a}$ on the complex torus $(\mathbb{C} \backslash 0)^{k}$ by $\mathbb{C}[A]$. Consider finite sets $A_{0}, \ldots, A_{l}$ in $\mathbb{Z}^{k}$, such that the dimension of the convex hull of $A_{0}+\cdots+A_{l}$ is not greater than $l$. Let $\Sigma$ be the set of all collections $\left(\varphi_{0}, \ldots, \varphi_{l}\right) \in \mathbb{C}\left[A_{0}\right] \oplus \cdots \oplus \mathbb{C}\left[A_{l}\right]$ such that $\varphi_{0}(y)=\cdots=\varphi_{l}(y)=0$ for some $y \in(\mathbb{C} \backslash 0)^{k}$.

If the closure of $\Sigma$ is a hypersurface in $\mathbb{C}\left[A_{0}\right] \oplus \cdots \oplus \mathbb{C}\left[A_{l}\right]$, then

(1) it is defined by the equation $G=0$ for a certain irreducible polynomial $G$ of positive degree.

(2) For a generic collection $\left(\varphi_{0}, \ldots, \varphi_{l}\right) \in \Sigma$, the set $\left\{y \mid \varphi_{0}(y)=\cdots=\varphi_{l}(y)=0\right\}$ can be represented as $J \cdot T$, where

$$
T=\left\{z \mid z^{a}=z^{b} \text { for all } a \text { and } b \text { in the affine span of } A_{0}+\cdots+A_{l}\right\}
$$

is a subtorus in $(\mathbb{C} \backslash 0)^{k}$, and $J \subset(\mathbb{C} \backslash 0)^{k} / T$ is a certain finite set, whose cardinality does not depend on the choice of $\left(\varphi_{0}, \ldots, \varphi_{l}\right)$; we denote this cardinality by $d\left(A_{0}, \ldots, A_{l}\right)$.

Definition 3.1 If the closure of $\Sigma$ is a hypersurface, then the polynomial $G^{d\left(A_{0}, \ldots, A_{l}\right)}$ is called the $\left(A_{0}, \ldots, A_{l}\right)$-resultant and is denoted by $R_{A_{0}, \ldots, A_{l}}$, otherwise we set $R_{A_{0}, \ldots, A_{l}}=1$ by definition. 
Remark The resultant is uniquely defined up to multiplication by a nonzero constant, and all equalities involving resultants should be understood correspondingly.

This definition differs from that of [25] and [14] by the exponent $d\left(A_{0}, \ldots, A_{l}\right)$. This exponent slightly simplifies computations and can be easily expressed in terms of $A$ as follows.

Multiplicity of the Resultant Let $A_{0}, \ldots, A_{k}$ be finite sets in $\mathbb{Z}^{k}$. The resultant $R_{A_{0}, \ldots, A_{k}}$ is by definition a certain power $d\left(A_{0}, \ldots, A_{k}\right)$ of an irreducible polynomial. Here we recall an explicit formula for the number $d\left(A_{0}, \ldots, A_{k}\right)$ and a criterion for triviality of the resultant $R_{A_{0}, \ldots, A_{k}}$ in terms of the sets $A_{0}, \ldots, A_{k}$.

Definition 3.2 The dimension of the convex hull of a finite set $A \subset \mathbb{R}^{k}$ is called the dimension of $A$ and is denoted by $\operatorname{dim} A$.

Definition 3.3 For every nonempty subset $J \subset\{0, \ldots, k\}$, the difference $\operatorname{dim} \sum_{j \in J} A_{j}-|J|$ is called the codimension of the collection $A_{j}, j \in J$, and is denoted by $\operatorname{codim} J$.

\section{Proposition 3.4 [25]}

(1) There exists $J$ with $\operatorname{codim} J<-1$ if and only if $R_{A_{0}, \ldots, A_{k}}=1$.

(2) If $\operatorname{codim} J \geqslant-1$ for every $J$, then there exists the minimal set $J_{0} \subset\{0, \ldots, k\}$, such that $\operatorname{codim} J_{0}=-1$.

Under the assumption of Proposition 3.4(2), let $L_{\mathbb{Z}} \subset \mathbb{Z}^{k+1}$ be the lattice generated by the set $\left(\sum_{j \in J_{0}} A_{j}\right) \times\{1\} \subset \mathbb{Z}^{k} \oplus \mathbb{Z}$, and let $L \subset \mathbb{R}^{k+1}$ be the linear span of $L_{\mathbb{Z}}$, denote the number $\left|\left(L \cap \mathbb{Z}^{k+1}\right) / L_{\mathbb{Z}}\right|$ by $d_{1}$.

Denote the projection $\mathbb{R}^{k+1} \rightarrow \mathbb{R}^{k+1} / L$ by $s$, and choose the volume form $\eta$ on $\mathbb{R}^{k+1} / L$ such that the volume of $\mathbb{R}^{k+1} /\left(L+\mathbb{Z}^{k+1}\right)$ equals $\left(k-\left|J_{0}\right|+1\right)$ !. Denote the $\eta$-mixed volume of the convex hulls of the sets $s\left(A_{j} \times\{1\}\right), j \in\{0, \ldots, k\} \backslash J_{0}$, by $d_{2}$.

Proposition 3.5 [10] In the notation above, $d\left(A_{0}, \ldots, A_{k}\right)=d_{1} \cdot d_{2}$.

$A$-determinant Let $l$ be the dimension of the affine span $L$ of a finite set $A \subset \mathbb{Z}^{k}$, let $t_{1}, \ldots, t_{k}$ be the standard coordinates on $\mathbb{R}^{k}$, and let $y_{1}, \ldots, y_{k}$ be the standard coordinates on $(\mathbb{C} \backslash 0)^{k}$. Choose numbers $i_{1}, \ldots, i_{l}$ such that the functions $t_{i_{1}}, \ldots, t_{i_{l}}$ form a system of coordinates on $L$ (for example, if $\operatorname{dim} A=k$, then $\left\{i_{1}, \ldots, i_{l}\right\}=$ $\{1, \ldots, k\})$.

Definition 3.6 The A-determinant is the polynomial $E_{A}$ on $\mathbb{C}[A]$, defined by the equality

$$
E_{A}(\varphi)=R_{A, \ldots, A}\left(\varphi, y_{i_{1}} \frac{\partial \varphi}{\partial y_{i_{1}}}, \ldots, y_{i_{l}} \frac{\partial \varphi}{\partial y_{i_{l}}}\right)
$$

Remark The A-determinant is uniquely defined up to multiplication by a nonzero constant, and does not depend on the choice of the collection $i_{1}, \ldots, i_{l}$, because 
changing this collection results in multiplication of the vector $\left(\varphi, y_{i_{1}} \frac{\partial \varphi}{\partial y_{i_{1}}}, \ldots, y_{i_{l}} \frac{\partial \varphi}{\partial y_{i_{l}}}\right)$ by a square nondegenerate matrix (by definition, the resultant $R_{A}, \ldots, A$ is invariant with respect to multiplication of its argument by a nondegenerate matrix).

\subsection{A-discriminants ([14])}

For a finite set $A \in \mathbb{Z}^{k}$, let $\Sigma_{A} \subset \mathbb{C}[A]$ be the set of all polynomials $\varphi \in \mathbb{C}[A]$ such that both $\varphi$ and its differential $\mathrm{d} \varphi$ vanish at some point $y \in(\mathbb{C} \backslash 0)^{k}$.

If the closure of $\Sigma_{A}$ is a hypersurface, then

(1) it is defined by the equation $G=0$ for a certain irreducible polynomial $G$ of positive degree.

(2) For a generic $\varphi \in \Sigma_{A}$, its singular locus $\{y \mid \varphi(y)=\mathrm{d} \varphi(y)=0\}$ has the form $J \cdot T$, where $T=\left\{z \mid z^{a}=z^{b}\right.$ for all $a$ and $b$ in the affine span of $\left.A\right\}$ is a subtorus in $(\mathbb{C} \backslash 0)^{k}$, and $J \subset(\mathbb{C} \backslash 0)^{k} / T$ is a finite set, whose cardinality $|J|$ does not depend on $\varphi$.

Definition 3.7 If the closure of $\Sigma_{A}$ is a hypersurface, then the polynomial $G^{|J|}$ is called the $A$-discriminant and is denoted by $D_{A}$; otherwise, we set $D_{A}=1$.

Remark The discriminant is uniquely defined up to multiplication by a nonzero constant, and all equalities involving discriminants should be understood correspondingly.

This definition differs from [14] by the exponent $|J|$. This exponent slightly simplifies computations in what follows, and can be easily expressed in terms of $A$ : let $L_{\mathbb{Z}} \subset \mathbb{Z}^{k+1}$ be the lattice generated by the set $A \times\{1\} \subset \mathbb{Z}^{k} \oplus \mathbb{Z}$, and let $L \subset \mathbb{R}^{k+1}$ be its linear span, then $|J|=\left|\left(L \cap \mathbb{Z}^{k+1}\right) / L_{\mathbb{Z}}\right|$.

Lemma 3.8 For every $A \subset \mathbb{Z}^{m}$, the discriminant $D_{A}$ is a power of an irreducible polynomial.

Proof The set of all pairs $(\varphi, y) \in \mathbb{C}[A] \times(\mathbb{C} \backslash 0)^{m}$, such that $\varphi(y)=\mathrm{d} \varphi(y)=0$, is the total space of a vector bundle $\mathcal{V}$ with the base space $(\mathbb{C} \backslash 0)^{m}$ and the projection $\mathbb{C}[A] \times(\mathbb{C} \backslash 0)^{m} \rightarrow(\mathbb{C} \backslash 0)^{m}$. Thus, it is irreducible, and thus its image $\Sigma_{A}$ under the projection to $\mathbb{C}[A]$ is also irreducible.

Recall that the dimension of the convex hull of $A \subset \mathbb{Z}^{k}$ is denoted by $\operatorname{dim} A$.

Lemma 3.9 For a generic $\varphi \in \mathbb{C}[A]$, the codimension of the set of all points $y \in$ $(\mathbb{C} \backslash 0)^{k}$, such that $\varphi(y)=\mathrm{d} \varphi(y)=0$, equals $\operatorname{dim} A-\operatorname{codim} \Sigma_{A}+1$.

Proof The fiber of the vector bundle $\mathcal{V}$, introduced in the previous proof, has codimension $1+\operatorname{dim} A$ in $\mathbb{C}[A]$.

$A$-discriminants and $A$-determinants are related as follows. For a polynomial $\varphi(y)=\sum_{a \in A} \varphi_{a} y^{a}$ and a face $A^{\prime}$ of the set $A$, denote the polynomial $\sum_{a \in A^{\prime}} \varphi_{a} y^{a}$ by $\varphi^{A^{\prime}}$. Recall that coefficients $c^{A^{\prime}, A}$ are introduced in Sect. 2.5. 
Proposition 3.10 [14]

$$
E_{A}(\varphi)=\prod_{A^{\prime}}\left(D_{A^{\prime}}\left(\varphi^{A^{\prime}}\right)\right)^{c^{A^{\prime}, A}},
$$

where $A^{\prime}$ runs over all faces of $A$, including $A^{\prime}=A$.

This also follows from Theorem 2.15, see Sect. 3.8.

\subsection{Dual Defectiveness}

Definition 3.11 A finite set $A \subset \mathbb{Z}^{k}$ is said to be dual defect, if $D_{A}=1$.

We recall a few simple facts about dual defect sets. There is also a well-known way to decide combinatorially if the set is dual defect or not (Corollary 5.13), which will follow from our results on Newton polyhedra of discriminants. One more prospective combinatorial criterion for dual defectiveness is given by Conjecture 3.20 below. Note that these facts do not provide classification of dual defect sets, which is a much more complicated problem, and is solved only for Delzant polytopes as of now, see [5]. An obvious but useful reformulation of this definition is as follows.

Lemma 3.12 A finite set $A \subset \mathbb{Z}^{k}$, whose convex hull is $k$-dimensional, is dual defect if and only if a generic polynomial in $\mathbb{C}[A]$ has a singular point.

Proof If a generic polynomial $\varphi \in \mathbb{C}[A]$ has a singular point, then a generic line of the form $\left\{\varphi-c \mid c \in \mathbb{C}\right.$ intersects the set $\Sigma_{A}$, thus codim $\Sigma_{A}=1$.

If codim $\Sigma_{A}=1$ and the convex hull of $A$ is $k$-dimensional, then a generic polynomial in $\Sigma_{A}$ has an isolated singular point, thus all nearby polynomials in $\Sigma_{A}$ have a singular point as well.

Proposition 3.13 (Monotonicity; see also [3]) If a subset $A^{\prime}$ of a finite set $A \subset \mathbb{Z}^{k}$ is not dual defect and is not contained in an affine hyperplane, then $A$ is not dual defect.

Proof We prove this by induction on $|A|$. Since dual defectiveness is by definition invariant with respect to parallel translations, the inductive step can be reduced to the following fact: if $A^{\prime}$ is not dual defect and is not contained in an affine hyperplane, then $A^{\prime} \cup\{0\}$ is not dual defect. The latter implication follows by Lemma 3.12

Dual defect sets are "thin" in many senses; for example, as in the following.

Proposition 3.14 If $A$ is not contained in a union of two parallel hyperplanes, then it is not dual defect.

This can be deduced from [4]; we also give a simple self-contained proof, which consists of two simple properties of iterated circuits. 
Definition 3.15 A set $B \subset \mathbb{R}^{m}$ of cardinality $m+2$ is called a circuit if none of its cardinality $m+1$ subsets is contained in an affine hyperplane.

A set $B \subset \mathbb{R}^{m}$ is called an iterated circuit if it is not contained in an affine hyperplane, and if, after a suitable parallel translation, it can be represented as a disjoint union $\{0\} \sqcup B_{1} \sqcup \cdots \sqcup B_{p}$, such that the following condition is satisfied: denote the linear span of $\{0\} \sqcup B_{1} \sqcup \cdots \sqcup B_{i}$ by $L_{i}$ for $i=0, \ldots, p$, then the projection $L_{i+1} \rightarrow L_{i+1} / L_{i}$ maps the union $\{0\} \sqcup B_{i+1}$ injectively onto a circuit in $L_{i+1} / L_{i}$.

The minimal possible number $p$ in this representation is called the depth of the iterated circuit $B$.

Example 3.16 Some iterated circuits do not contain circuits. The simplest example is the set of integer points in the unit ball centered at the origin in $\mathbb{R}^{m}$ (this set is an iterated circuit: the sets $B_{i}$ above are the pairs of its opposite points). If the cardinality of $B \subset \mathbb{R}^{m}$ is $2 m$ or greater, then it is not an iterated circuit.

Lemma 3.17 An iterated circuit $B \subset \mathbb{Z}^{m}$ is not dual defect.

Proof Proving this by induction on the depth of $B$, the inductive step can be reduced to the following statement by a suitable $\mathbb{Q}$-affine change of coordinates in $\mathbb{R}^{m}$ : if $B \subset \mathbb{Z}^{k}$ is not dual defect and $B^{\prime} \sqcup\{0\} \subset \mathbb{Z}^{l}$ is a circuit, then $B \sqcup B^{\prime} \subset \mathbb{Z}^{k} \oplus \mathbb{Z}^{l}$ is not dual defect.

Since $B^{\prime} \sqcup\{0\}$ is a circuit and $B$ is not dual defect, then generic polynomials $\varphi \in \mathbb{C}[B]$ and $\psi \in \mathbb{C}\left[B^{\prime}\right]$ have singular points by Lemma 3.12. Thus, their sum $\varphi+\psi$, which is a generic polynomial in $\mathbb{C}\left[B \sqcup B^{\prime}\right]$, has a singular point as well, hence $B \sqcup B^{\prime}$ is not dual defect by the same lemma.

Lemma 3.18 If a finite set $B \subset \mathbb{R}^{m}$ does not contain an iterated circuit, then it is contained in a union of two parallel hyperplanes.

Example 3.19 If $B$ does not contain a circuit, it does not imply that $B$ is contained in a union of two parallel hyperplanes. The simplest example is the same as the previous one.

Proof Without loss of generality, we may assume that $0 \in B$, and pick a maximal subset $B^{\prime} \subset B$, such that

(1) $0 \in B^{\prime}$, and

(2) $B^{\prime}$ is an iterated circuit as a subset of its vector span $L$

The image $\widetilde{B}$ of the set $B$ under the projection along $L$ consists of at most $k-$ $\operatorname{dim} L+1$ points, otherwise $B$ is not maximal. Thus, $\widetilde{B}$ is contained in the union of two parallel hyperplanes, and so is $B$.

Proof of Proposition 3.14 If $A$ is dual defect, then it does not contain an iterated circuit by Proposition 3.13 and Lemma 3.17, and thus it is contained in the union of two parallel hyperplanes by Lemma 3.18.

In particular, we have proved the easy half of the following conjecture. 
Conjecture 3.20 A finite set $A \subset \mathbb{Z}^{k}$, whose convex hull is $k$-dimensional, is dual defect if and only if it does not contain an iterated circuit.

Recall that $D_{A}$ is a polynomial in the indeterminate coefficients $\varphi_{a}$ of the polyno$\operatorname{mial} \varphi(y)=\sum_{a \in A} \varphi_{a} y^{a}$.

Lemma 3.21 For every non-dual defect $A \subset \mathbb{Z}^{m}$ and every $a \in A$, the discriminant $D_{A}$ has positive degree as a polynomial of $\varphi_{a}$, unless it is a constant.

Proof Since dual defectiveness is by definition invariant with respect to parallel translations, it is enough to prove the statement for $a=0$, which follows from Lemma 3.12.

\subsection{Discriminants $D_{A_{0}, \ldots, A_{l}}$ and $B_{A_{0}, \ldots, A_{l}}$}

Discriminant $D_{A_{0}, \ldots, A_{l}}$ Let $A_{0}, \ldots, A_{l}, l \leqslant k$, be finite sets in $\mathbb{Z}^{k}$, and let $\Sigma_{A_{0}, \ldots, A_{l}} \subset \mathbb{C}\left[A_{0}\right] \oplus \cdots \oplus \mathbb{C}\left[A_{l}\right]$ be the set of all collections of polynomials $\left(\varphi_{0}, \ldots, \varphi_{l}\right)$, such that their differentials are linearly dependent at some point of the set $\left\{y \in(\mathbb{C} \backslash 0)^{k} \mid \varphi_{0}(y)=\cdots=\varphi_{l}(y)=0\right\}$. The union of the codimension 1 components of the closure $\overline{\Sigma_{A_{0}, \ldots, A_{l}}}$ is defined by the equation $G=0$ for a certain square-free polynomial $G$ on $\mathbb{C}\left[A_{0}\right] \oplus \cdots \oplus \mathbb{C}\left[A_{l}\right]$.

Definition 3.22 The polynomial $G$ is called the reduced A-discriminant of degree $\left(A_{0}, \ldots, A_{l}\right)$ and is denoted by $D_{A_{0}, \ldots, A_{l}}^{\text {red }}$.

We assume that $l \leqslant k$, because the codimension of $\Sigma_{A_{0}, \ldots, A_{l}}$ is greater than 1 otherwise (however, one can still study the tropicalization of $\Sigma_{A_{0}, \ldots, A_{l}}$ instead of its Newton polyhedron; see [6] and [26] for details).

If $l=k$, then $D_{A_{0}, \ldots, A_{k}}^{\text {red }}$ is the reduced version of the resultant $R_{A_{0}, \ldots, A_{k}}$ (see Definition 3.1); if $l=0$, then $D_{A_{0}}^{\text {red }}$ is the reduced version of the discriminant $D_{A_{0}}$ (see Definition 3.7). In both of these cases, the discriminant set $\Sigma_{A_{0}, \ldots, A_{l}}$ is irreducible, and a combinatorial way to verify $\operatorname{codim} \Sigma_{A_{0}, \ldots, A_{l}}=1$ is known (see Corollary 5.13(2) for $l=0$ and Proposition 3.4(1) for $l=k$ ). In general, however, unlike in these two special cases, the set $\Sigma_{A_{0}, \ldots, A_{l}}$ may be not irreducible and not even of pure dimension. Thus, both its codimension 1 part $\left\{D_{A_{0}, \ldots, A_{l}}^{\text {red }}=0\right\}$ and its higher codimension part $\Sigma_{A_{0}, \ldots, A_{l}} \backslash\left\{D_{A_{0}, \ldots, A_{l}}^{\text {red }}=0\right\}$ may be nonempty for $0<l<k$ (see Example 3.25 below). Nevertheless, restricting our attention to the codimension 1 components, it turns out to be possible to express $D_{A_{0}, \ldots, A_{l}}^{\text {red }}$ in terms of $A$-discriminants by means of the Cayley trick, see Theorem 3.31 below.

\section{Dual Defectiveness}

Definition 3.23 A collection of sets $A_{0}, \ldots, A_{l}$ is said to be dual defect if the closure of the set $\Sigma_{A_{0}, \ldots, A_{l}}$ is not a hypersurface.

One sufficient condition for non-dual defectiveness is as follows. 
Proposition 3.24 If neither of the sets $A_{0}, \ldots, A_{l}$ is contained in an affine hyperplane, and at least one of them is not dual defect, then the collection $A_{0}, \ldots, A_{l}$ is not dual defect.

The proof is given in Sect. 3.6 below, as well as a more refined condition (Proposition 3.40), which is presumably a criterion. Note that neither of the conditions of this statement can be omitted in general, as the following examples demonstrate.

Example 3.25 If $k=2$ and $A_{0}=A_{1}$ is the set of vertices of the standard 2-dimensional simplex (which is dual defect), then $\Sigma_{A_{0}, A_{1}}$ has codimension 2 .

If $k=2, A_{0}=\{0,1,2\} \times\{0\}$, and $A_{1}=\{0,1\} \times\{0,1\}$ (neither of these sets is dual defect, but $\operatorname{dim} A_{0}<2$ ), then $\overline{\Sigma_{A_{0}, A_{1}}}$ has two components. The first one consists of all pairs of polynomials of the form $\left(c(x-a)^{2}, b_{11} x y+b_{01} x+b_{10} y+b_{00}\right)$, and has codimension 1 . Another one consists of all pairs of polynomials of the form $\left(c_{1}\left(x-a_{1}\right)\left(x-a_{2}\right), c_{2}\left(x-a_{1}\right)(y-b)\right)$, and has codimension 2.

Discriminant $B_{A_{0}, \ldots, A_{l}}$ We also consider another possible definition of discriminant, such that the corresponding version of the non-dual defectiveness assumption is weaker than the conventional one. Let $W$ be the set of all collections $\left(y, \varphi_{0}, \ldots, \varphi_{l}\right) \in(\mathbb{C} \backslash 0)^{k} \times \mathbb{C}\left[A_{0}\right] \times \cdots \times \mathbb{C}\left[A_{l}\right]$, such that $\varphi_{0}(y)=\cdots=\varphi_{l}(y)=0$. Let $S_{A_{0}, \ldots, A_{l}} \subset \mathbb{C}\left[A_{0}\right] \times \cdots \times \mathbb{C}\left[A_{l}\right]$ be the minimal (closed) set, such that the projection $W \rightarrow \mathbb{C}\left[A_{0}\right] \times \cdots \times \mathbb{C}\left[A_{l}\right]$ is a fiber bundle outside of $S$.

Definition 3.26 The set $S_{A_{0}, \ldots, A_{l}}$ is called the bifurcation set. The collection $A_{0}, \ldots, A_{l}$ is said to be $B$-nondegenerate if $S_{A_{0}, \ldots, A_{l}}$ is a hypersurface. In this case, the equation of $S_{A_{0}, \ldots, A_{l}}$ is denoted by $B_{A_{0}, \ldots, A_{l}}$ and is called the bifurcation discriminant.

In contrast to the discriminant $D_{A_{0}, \ldots, A_{l}}^{\text {red }}$, the bifurcation discriminant takes "singularities of the system $\varphi_{0}=\cdots=\varphi_{l}=0$ at infinity" into account.

Example 3.27 In the notation of the previous example $\left(k=2, A_{0}=\{0,1,2\} \times\right.$ $\left.\{0\}, A_{1}=\{0,1\} \times\{0,1\}\right)$, despite the fact that the discriminant set $\overline{\Sigma_{A_{0}, A_{1}}}$ is not of pure dimension, the bifurcation set $S_{A_{0}, A_{1}} \supset \overline{\Sigma_{A_{0}, A_{1}}}$ is a hypersurface that consists of five components. A generic point in each of these components is as follows (the codimension 2 component of the discriminant set is the intersection of the last two components):

$$
\begin{aligned}
& c(x-a)^{2}, \quad b_{11} x y+b_{10} y+b_{01} x+b_{00} ; \\
& a_{1} x^{2}-a_{2} x, \quad b_{11} x y+b_{10} y+b_{01} x+b_{00} ; \\
& a_{1} x-a_{2}, \quad b_{11} x y+b_{10} y+b_{01} x+b_{00} ; \\
& c_{1}\left(x-a_{1}\right)\left(x-a_{2}\right), \quad c_{2}\left(x y-a_{1} y+b_{01} x+b_{00}\right) ; \\
& c_{1}\left(x-a_{1}\right)\left(x-a_{2}\right), \quad c_{2}\left(b_{11} x y+b_{10} y+x-a_{1}\right) .
\end{aligned}
$$


In particular, we have

$$
B_{A_{0}, A_{1}}=D_{A_{0}, A_{1}}^{\mathrm{red}} \cdot D_{\{(0,0)\}, A_{1}}^{\mathrm{red}} \cdot D_{\{(2,0)\}, A_{1}}^{\mathrm{red}} \cdot D_{A_{0},\{0,1\} \times\{1\}}^{\mathrm{red}} \cdot D_{A_{0},\{0,1\} \times\{0\}}^{\mathrm{red}} \cdot
$$

These observations generalize as follows.

Conjecture 3.28 All collections are B-nondegenerate.

Proposition 3.29 If the convex hulls of finite sets $A_{0}, \ldots, A_{l}$ in $\mathbb{Z}^{k}$ have the same dual fan, then the collection $A_{0}, \ldots, A_{l}$ is $B$-nondegenerate.

In particular, if a collection consists of one set, then it is $B$-nondegenerate, in contrast to dual defectiveness. The proof is given in Sect. 3.7.

Lemma 3.30 The bifurcation discriminant $B_{A_{0}, \ldots, A_{l}}$ equals the product of discriminants $D_{A_{0}^{\prime}, \ldots, A_{l}^{\prime}}^{\text {red }}$ over all compatible collections of faces $A_{0}^{\prime} \subset A_{0}, \ldots, A_{l}^{\prime} \subset A_{l}$.

We omit the proof, since it follows by definitions.

\subsection{Cayley Trick}

Let $e_{0}, \ldots, e_{l}$ be the standard basis in $\mathbb{Z}^{l+1}$. For $J \subset\{0, \ldots, l\}$, denote the set $\bigcup_{j \in J} A_{j} \times\left\{e_{j}\right\}$ by $A_{J} \subset \mathbb{Z}^{k} \oplus \mathbb{Z}^{l+1}$. We identify the space $\bigoplus_{j \in J} \mathbb{C}\left[A_{j}\right]$ with the space $\mathbb{C}\left[A_{J}\right]$ by identifying a collection of polynomials $\varphi_{j} \in \mathbb{C}\left[A_{j}\right], j \in J$, on the complex torus $(\mathbb{C} \backslash 0)^{n}$ with the polynomial $\sum_{j \in J} \lambda_{j} \varphi_{j}$ on $(\mathbb{C} \backslash 0)^{k} \times(\mathbb{C} \backslash 0)^{l+1}$, where $\lambda_{0}, \ldots, \lambda_{l}$ are the standard coordinates on $(\mathbb{C} \backslash 0)^{l+1}$. This identification allows us to regard the discriminant $D_{A_{J}}^{\mathrm{red}}$ as a polynomial on the space $\mathbb{C}\left[A_{0}\right] \oplus \cdots \oplus \mathbb{C}\left[A_{l}\right]$.

Theorem 3.31 (Cayley trick) The discriminant $D_{A_{0}, \ldots, A_{l}}^{\mathrm{red}}$ equals the product of the discriminants $D_{A_{J}}^{\text {red }}$ over all subsets $J \subset\{0, \ldots, l\}$, such that $\operatorname{codim} J \leqslant \operatorname{codim} J^{\prime}$ for every $J^{\prime} \supset J$ (recall that codim $J$ stands for the difference $\operatorname{dim} \sum_{j \in J} A_{j}-|J|$ ).

For $l=k$, this is proved in [14]; the only admissible $J$ is $\{0, \ldots, k\}$ in this case. If neither of $A_{i}$ is contained in an affine hyperplane, then $\{0, \ldots, k\}$ is the only admissible $J$ as well. The proof of Theorem 3.31 is given at the end of this subsection and is based on the following construction.

Definition 3.32 We define $\Sigma_{\left\{j_{0}, \ldots, j_{q}\right\}}$ as the set of all collections $\left(\varphi_{0}, \ldots, \varphi_{l}\right) \subset$ $\mathbb{C}\left[A_{0}\right] \oplus \cdots \oplus \mathbb{C}\left[A_{l}\right]$, such that

(1) $\varphi_{0}(x)=\cdots=\varphi_{l}(x)=0$ for some $x \in(\mathbb{C} \backslash 0)^{k}$, and

(2) $\lambda_{j_{0}} \mathrm{~d} \varphi_{j_{0}}(x)+\cdots+\lambda_{j_{q}} \mathrm{~d} \varphi_{j_{q}}(x)=0$ for some $\left(\lambda_{j_{0}}, \ldots, \lambda_{j_{q}}\right) \in(\mathbb{C} \backslash 0)^{q+1}$

We have two options for each set $\Sigma_{J}$ : 


\section{Lemma 3.33}

(A) If $A_{J}$ is not dual defect, and $\operatorname{codim} J \leqslant \operatorname{codim} J^{\prime}$ for every $J^{\prime} \supset J$, then the closure of $\Sigma_{J}$ is defined by the equation $D_{A_{J}}^{\mathrm{red}}=0$.

(B) Otherwise, codim $\Sigma_{J}>1$.

The proof of this lemma is given below and is based on the following important fact.

Lemma 3.34 [19] Generic polynomials $\psi_{i} \in \mathbb{C}\left[A_{i}\right], ; i=0, \ldots, l$, have a common root in $(\mathbb{C} \backslash 0)^{n}$ if and only if $\operatorname{dim} A_{i_{1}}+\cdots+A_{i_{q}} \geqslant q$ for every sequence $0 \leqslant i_{1}<$ $\cdots<i_{q} \leqslant l$.

This fact is mentioned as obvious in [19], but we prefer to give a proof for the sake of completeness.

Proof If $l \geqslant k$ then the statement is obvious, because, on one hand, generic polynomials $\psi_{0}, \ldots, \psi_{l}$ have no common roots and, on the other hand, we have $\operatorname{dim} A_{0}+\cdots+$ $A_{l}<l+1$. The case $l<k-1$ can be reduced to the case $l=k-1$ by introducing arbitrary finite sets $A_{l+1}, \ldots, A_{k-1}$, whose convex hulls are $k$-dimensional, and considering generic polynomials $\psi_{i} \in \mathbb{C}\left[A_{i}\right], i=0, \ldots, k-1$. Finally, if $l=k-1$, then the number of common roots of generic polynomials $\psi_{0}, \ldots, \psi_{k-1}$ equals $k$ ! times the mixed volume of the convex hulls of the sets $A_{0}, \ldots, A_{k-1}$, which is nonzero under the assumption of the lemma by Lemma 2.2.

Proof of Lemma 3.33 First, note that the image of the set $\Sigma_{J}$ under the natural projection $\mathbb{C}\left[A_{0}\right] \oplus \cdots \oplus \mathbb{C}\left[A_{l}\right] \rightarrow \bigoplus_{j \in J} \mathbb{C}\left[A_{j}\right]$ is contained in $\Sigma_{A_{J}}$. In particular, if $\operatorname{codim} \Sigma_{A_{J}}>1$, then the set $\Sigma_{J}$ satisfies both of the statements (A) and (B) of Lemma 3.33, independently of codim $J^{\prime}$ for $J^{\prime} \supset J$. Thus, we can assume that $\operatorname{codim} \Sigma_{A_{J}}=1$, i.e., $A_{J}$ is not dual defect.

Consider the vector space $L \subset \mathbb{R}^{n}$, parallel to the affine span of the sum $\sum_{j \in J} A_{j}$, denote the projection $\mathbb{R}^{k} \rightarrow \mathbb{R}^{k} / L$ by $p_{L}$, and consider the torus $T_{L}=\left\{x \mid x^{a}=\right.$ 1 for $a \in L\} \subset(\mathbb{C} \backslash 0)^{k}$. Then, for a generic polynomial in $\mathbb{C}\left[A_{j}\right]$, its restriction to $T_{L}$ is a generic polynomial in $\mathbb{C}\left[p_{L} A_{j}\right]$. Thus, by Lemma 3.34, generic polynomials $\varphi_{i} \in \mathbb{C}\left[A_{i}\right], i \notin J$, have a common zero on a torus $c \cdot T_{L}, c \in(\mathbb{C} \backslash 0)^{k}$, if and only if every subset $I \subset\{0, \ldots, l\} \backslash J$ satisfies inequality $\operatorname{dim} \sum_{i \in I} p_{L}\left(A_{i}\right) \geqslant|I|$, i.e., $\operatorname{codim} I \cup J \geqslant \operatorname{codim} J$.

Proof of Part $A$ For $J=\left\{j_{0}, \ldots, j_{q}\right\}$ and a polynomial $\left.\lambda_{j_{0}} \varphi_{j_{0}}+\cdots+\lambda_{j_{q}} \varphi_{j_{q}}\right) \in$ $\mathbb{C}\left[A_{J}\right]$, the set

$$
\begin{aligned}
& \left\{x \in(\mathbb{C} \backslash 0)^{k} \mid \varphi_{j_{0}}(x)=\cdots=\varphi_{j_{q}}(x)=0 \text { and } \lambda_{j_{0}} \mathrm{~d} \varphi_{j_{0}}(x)+\cdots+\lambda_{j_{q}} \mathrm{~d} \varphi_{j_{q}}(x)=0\right. \\
& \text { for some } \left.\left(\lambda_{j_{0}}, \ldots, \lambda_{j_{q}}\right) \in(\mathbb{C} \backslash 0)^{q+1}\right\}
\end{aligned}
$$

is nonempty and preserved under multiplication by elements of $T_{L}$, and thus it contains a torus $c \cdot T_{L}, c \in(\mathbb{C} \backslash 0)^{k}$. Thus, generic polynomials $\varphi_{i} \in \mathbb{C}\left[A_{i}\right], i \notin J$, have a common zero on it under the assumption of Part A. Thus, every fiber of the projection $\Sigma_{J} \rightarrow \Sigma_{A_{J}}$ is Zariski open in the corresponding fiber of the ambient projection $\mathbb{C}\left[A_{0}\right] \oplus \cdots \oplus \mathbb{C}\left[A_{l}\right] \rightarrow \bigoplus_{j \in J} \mathbb{C}\left[A_{j}\right]$. Thus, codim $\Sigma_{J}=\operatorname{codim} \Sigma_{A_{J}}=1$. 
Proof of Part $B \quad$ For $J=\left\{j_{0}, \ldots, j_{q}\right\}$ and a generic polynomial $\lambda_{j_{0}} \varphi_{j_{0}}+\cdots+\lambda_{j_{q}} \varphi_{j_{q}}$ in $\mathbb{C}\left[A_{J}\right]$, the set

$$
\begin{aligned}
& \left\{x \in(\mathbb{C} \backslash 0)^{k} \mid \varphi_{j_{0}}(x)=\cdots=\varphi_{j_{q}}(x)=0 \text { and } \lambda_{j_{0}} \mathrm{~d} \varphi_{j_{0}}(x)+\cdots+\lambda_{j_{q}} \mathrm{~d} \varphi_{j_{q}}(x)=0\right. \\
& \text { for some } \left.\left(\lambda_{j_{0}}, \ldots, \lambda_{j_{q}}\right) \in(\mathbb{C} \backslash 0)^{q+1}\right\}
\end{aligned}
$$

consists of a finite number of tori $c_{s} \cdot T_{L}, c_{s} \in(\mathbb{C} \backslash 0)^{k}, s=1, \ldots, S$, because $A_{J}$ is not dual defect. Thus, generic polynomials $\varphi_{i} \in \mathbb{C}\left[A_{i}\right], i \notin J$, have no common zero on it under the assumption of Part B. Thus, a generic fiber of the projection $\Sigma_{J} \rightarrow \Sigma_{A_{J}}$ has codimension 1 or greater in the corresponding fiber of the ambient projection $\mathbb{C}\left[A_{0}\right] \oplus \cdots \oplus \mathbb{C}\left[A_{l}\right] \rightarrow \bigoplus_{j \in J} \mathbb{C}\left[A_{j}\right]$. Thus, codim $\Sigma_{J} \geqslant \operatorname{codim} \Sigma_{A_{J}}>1$.

Proof of Theorem 3.31 The desired set $\Sigma_{A_{0}, \ldots, A_{l}}$ is the union of the sets $\Sigma_{J}$ over all $J \subset\{0, \ldots, l\}$. By Lemma 3.33, the product of the discriminants, mentioned in the formulation of the theorem, vanishes at the union of codimension 1 components of the closure $\overline{\Sigma_{A_{0}, \ldots, A_{l}}}$. Since all nonconstant discriminants in this product are irreducible and distinct by Lemmas 3.8 and 3.21, this product is a square-free polynomial and thus equals $D_{A_{0}, \ldots, A_{l}}^{\text {red }}$.

\subsection{Proof of Proposition 3.24}

The proof relies upon Lemma 3.33 and relevant notation from the previous subsection. We split Proposition 3.24 into the following two lemmas.

Lemma 3.35 If $A_{0}$ is not dual defect, and $\operatorname{dim} A_{i}=k$ for every $i=0, \ldots, l$, then $A_{\{0, \ldots, l\}}$ is not dual defect.

Lemma 3.36 If $A_{\{0, \ldots, l\}}$ is not dual defect, and $\operatorname{dim} A_{i}=k$ for every $i=0, \ldots, l$, then $\Sigma_{A_{0}, \ldots, A_{l}}$ is irreducible of codimension 1.

Proof of both of these lemmas is given below and is based on the following fact.

Lemma 3.37 If $\operatorname{dim} A=k, \varphi \in \mathbb{C}[A], \varphi\left(y_{0}\right)=0$, and a vector $v \in \mathbb{C}^{n}$ is close enough to $\mathrm{d} \varphi\left(y_{0}\right)$, then there exists $\tilde{\varphi} \in \mathbb{C}[A]$ near $\varphi$, such that $\tilde{\varphi}\left(y_{0}\right)=0$ and $\mathrm{d} \tilde{\varphi}\left(y_{0}\right)=v$.

Proof One can readily verify this statement if $A$ is of cardinality $k+1$ and $\varphi=0$. Thus, if $A_{0} \subset A$ is the set of vertices of a $k$-dimensional simplex, then there exists a small $\psi \in \mathbb{C}\left[A_{0}\right]$ such that $\mathrm{d} \psi\left(y_{0}\right)=v-\mathrm{d} \varphi\left(y_{0}\right)$, and we can set $\tilde{\varphi}=\varphi+\psi$.

Proof of Lemma 3.35 Since dual defectiveness is preserved by parallel translations, we can assume without loss of generality that $0 \in A_{0}$. By Lemma 3.9, a generic polynomial $\varphi_{0} \in \Sigma_{A_{0}}$ has an isolated singular point $y_{0}$. By Lemma 3.37, generic polynomials $\varphi_{i} \in \mathbb{C}\left[A_{i}\right]$, such that $\varphi_{i}\left(y_{0}\right)=0$, define a nondegenerate complete intersection $\varphi_{1}=\cdots=\varphi_{l}=0$, passing through $y_{0}$ and transversal to the hypersurface $\varphi_{0}=0$ in a punctured neighborhood of $y_{0}$. Then, for generic $\tilde{\varphi}_{i} \in \mathbb{C}\left[A_{i}\right]$ near $\varphi_{i}, i=0, \ldots, l$, 
there exists a small number $\varepsilon$ such that the nondegenerate complete intersection $\tilde{\varphi}_{1}=\cdots=\tilde{\varphi}_{l}=0$ is tangent to the smooth hypersurface $\tilde{\varphi}_{0}=\varepsilon$ at some point near $y_{0}$, which implies that $\left(\tilde{\varphi}_{0}-\varepsilon, \tilde{\varphi}_{1}, \ldots, \tilde{\varphi}_{l}\right) \in \Sigma_{A_{0}, \ldots, A_{l}}$. Thus, codim $\Sigma_{A_{0}, \ldots, A_{l}}=1$ near the point $\left(\varphi_{0}, \ldots, \varphi_{l}\right)$.

Since codim $\Sigma_{A_{0}, \ldots, A_{l}}=1$ at some point of this set,

$$
\Sigma_{A_{0}, \ldots, A_{l}}=\bigcup_{J \subset\{0, \ldots, l\}} \Sigma_{J},
$$

$\operatorname{codim} \Sigma_{J}>1$ for $J \varsubsetneqq\{0, \ldots, l\}$ by Lemma 3.33(B),

and $\Sigma_{\{0, \ldots, l\}}=\Sigma_{A_{\{0, \ldots, l\}}}$,

we have codim $\Sigma_{A_{\{0, \ldots, l\}}}=1$, thus $A_{\{0, \ldots, l\}}$ is not dual defect.

Proof of Lemma $3.36 \mathrm{~F}$ or every collection $\left(\varphi_{0}, \ldots, \varphi_{l}\right) \in \Sigma_{A_{0}, \ldots, A_{l}}$, there exists a point $y_{0}$, such that $\varphi_{0}\left(y_{0}\right)=\cdots=\varphi_{l}\left(y_{0}\right)=0$ and $\mathrm{d} \varphi_{0}\left(y_{0}\right), \ldots, \mathrm{d} \varphi_{l}\left(y_{0}\right)$ are linearly dependent. There exist vectors $v_{0}, \ldots, v_{l}$ near $\mathrm{d} \varphi_{0}\left(y_{0}\right), \ldots, \mathrm{d} \varphi_{l}\left(y_{0}\right)$, such that $\sum_{j=0}^{l} \lambda_{j} v_{j}=0$ with $\lambda_{j} \neq 0$ for every $j=0, \ldots, l$. Thus, by Lemma 3.37, there exists a collection $\left(\tilde{\varphi}_{0}, \ldots, \tilde{\varphi}_{l}\right)$ near $\left(\varphi_{0}, \ldots, \varphi_{l}\right)$, such that

$$
\sum_{j=0}^{l} \lambda_{j} \mathrm{~d} \tilde{\varphi}_{j}\left(y_{0}\right)=0
$$

with $\lambda_{j} \neq 0$ for every $j=0, \ldots, l$. This means that $\left(\lambda_{0}, \ldots, \lambda_{l}, y_{0}\right) \in \Sigma_{A_{\{0, \ldots, l\}}}$. Thus, $\Sigma_{A_{0}, \ldots, A_{l}}$ contains the irreducible codimension 1 set $\Sigma_{A_{\{0, \ldots, l\}}}$ and is contained in its closure, which completes the proof.

Refinement of Proposition 3.24 Consider finite sets $A_{0}, \ldots, A_{l}$ in $\mathbb{Z}^{k}$ and a subset $J \subset\{0, \ldots, l\}$.

Definition 3.38 $J$ is said to be reliable if $A_{J}$ is not dual defect, and $\operatorname{codim} J \leqslant$ $\operatorname{codim} J^{\prime}$ for every $J^{\prime} \supset J$ (recall that $\operatorname{codim} J$ stands for the difference $\left.\operatorname{dim} \sum_{j \in J} A_{j}-|J|\right)$.

Note that $J$ is reliable if and only if $\Sigma_{J}$ is a hypersurface.

Definition 3.39 Vectors $v_{0}, \ldots, v_{l}$ are said to be $J$-linearly dependent if $\sum_{j \in J} \lambda_{j} v_{j}=0$ with $\lambda_{j} \neq 0$ for every $j \in J$.

For a set $A \subset \mathbb{R}^{k}$, denote the space of all linear functions, whose restrictions to $A$ are constant, by $A^{\perp} \subset\left(\mathbb{R}^{k}\right)^{*}$. The following refined version of Proposition 3.24 is presumably a criterion for non-dual defectiveness of a collection of sets.

Proposition 3.40 A collection of finite sets $A_{0}, \ldots, A_{l}$ in $\mathbb{Z}^{k}$ is not dual defect, if codim $J \geqslant-1$ for every $J \subset\{0, \ldots, l\}$, and there exists a $J$-linearly dependent collection $\tilde{v}_{0} \in A_{0}^{\perp}, \ldots, \tilde{v}_{l} \in A_{l}^{\perp}$ with a reliable $J$ in every neighborhood of every linearly dependent collection of vectors $v_{0} \in A_{0}^{\perp}, \ldots, v_{l} \in A_{l}^{\perp}$. 
We can formulate Lemma 3.37 with no assumption $\operatorname{dim} A=k$ as follows. Denote the natural identification $T_{y}(\mathbb{C} \backslash 0)^{n} \rightarrow T_{(1, \ldots, 1)}(\mathbb{C} \backslash 0)^{n}$ by $e_{y}$.

Lemma 3.41 Suppose that $\varphi \in \mathbb{C}[A], \varphi(y)=0$, and $v \in \mathbb{C}^{n}$ is near $\mathrm{d} \varphi(y)$. Then every neighborhood of $\varphi$ contains $\tilde{\varphi} \in \mathbb{C}[A]$ with $\tilde{\varphi}(y)=0$ and $\mathrm{d} \tilde{\varphi}(y)=v$ if and only if $e_{y}(v) \in A^{\perp}$.

Proof of Proposition 3.40 For a collection $\left(\varphi_{0}, \ldots, \varphi_{l}\right) \subset \Sigma_{A_{0}, \ldots, A_{l}}$, choose a point $y$, such that $\varphi_{0}(y)=\cdots=\varphi_{l}(y)=0$ and the differentials $\mathrm{d} \varphi_{0}(y), \ldots, \mathrm{d} \varphi_{l}(y)$ are linearly dependent, and choose nearby vectors $v_{0}, \ldots, v_{l}$ to be $J$-linearly dependent for a reliable $J$. By Lemma 3.41, we have $\tilde{\varphi}_{i}(y)=0$ and $\mathrm{d} \tilde{\varphi}_{i}(y)=v_{i}$ for some $\tilde{\varphi}_{i}$ near $\varphi_{i}$; thus the collection $\left(\tilde{\varphi}_{0}, \ldots, \tilde{\varphi}_{l}\right)$ is contained in the hypersurface $\Sigma_{J}$.

\subsection{Proof of Proposition 3.29}

See Sect. 3.5 for the definition of $A_{J}$ and $\Sigma_{J}$ for $J \subset\{0, \ldots, l\}$.

We prove the statement by induction on $k$. For a subset $A^{\prime} \subset A$, there is a natural projection from $\mathbb{C}[A]$ to $\mathbb{C}\left[A^{\prime}\right]$ that assigns the polynomial $\sum_{a \in A^{\prime}} c_{a} y^{a}$ to a polynomial $\sum_{a \in A} c_{a} y^{a}$. For subsets $A_{0}^{\prime} \subset A_{0}, \ldots, A_{l}^{\prime} \subset A_{l}$, we denote the preimage of the set $B \subset \mathbb{C}\left[A_{0}^{\prime}\right] \oplus \cdots \oplus \mathbb{C}\left[A_{l}^{\prime}\right]$ under this projection by $\widetilde{B}$.

The bifurcation set $S_{A_{0}, \ldots, A_{l}}$ contains the set $\widetilde{S}_{A_{0}^{\prime}, \ldots, A_{l}^{\prime}}$ for every collection of compatible faces $A_{0}^{\prime} \subset A_{0}, \ldots, A_{l}^{\prime} \subset A_{l}$. The difference of $S_{A_{0}, \ldots, A_{l}}$ and $\bigcup_{A_{0}^{\prime}, \ldots, A_{l}^{\prime}} \widetilde{S}_{A_{0}^{\prime}, \ldots, A_{l}^{\prime}}$ is contained in the set $\Sigma_{A_{0}, \ldots, A_{l}}$, which is the union of irreducible sets $\Sigma_{J}, J \subset\{0, \ldots, l\}$. Thus, we can reformulate Proposition 3.29 as follows: every set of the form $\Sigma_{J}$ is contained in the closure of a set of the form $\Sigma_{J}$ or $\widetilde{S}_{A_{0}^{\prime}, \ldots, A_{l}^{\prime}}$, whose codimension is 1 .

For a generic point $\left(\varphi_{0}, \ldots, \varphi_{l}\right) \in \Sigma_{J}$, we have the following three cases:

(1) The set of all $y \in(\mathbb{C} \backslash 0)^{k}$, such that $\mathrm{d} \varphi_{0}(y), \ldots, \mathrm{d} \varphi_{l}(y)$ are linearly dependent, has positive dimension. Then it contains a germ of a curve $(\mathbb{C} \backslash 0) \rightarrow(\mathbb{C} \backslash 0)^{k}$, whose leading term is $\left(c_{1} t^{\gamma_{1}}, \ldots, c_{k} t^{\gamma_{k}}\right) \neq$ const. The covector $\left(\gamma_{1}, \ldots, \gamma_{k}\right) \neq 0$, as a function on $A_{i}$, attains its maximum at some proper face $A_{i}^{\prime} \subsetneq A_{i}$. Thus, $\left(\varphi_{0}, \ldots, \varphi_{l}\right) \in \widetilde{S}_{A_{0}^{\prime}, \ldots, A_{l}^{\prime}}$, which is a hypersurface by induction.

(2) $J=\{0, \ldots, l\}$, and there exists an isolated point $y \in(\mathbb{C} \backslash 0)^{k}$, such that $\mathrm{d} \varphi_{0}(y), \ldots, \mathrm{d} \varphi_{l}(y)$ are linearly dependent. Then we can choose vectors $v_{i}$ near $\mathrm{d} \varphi_{i}(y)$, such that the nontrivial linear combination of $v_{0}, \ldots, v_{l}$ is unique and has all nonzero coefficients. By Lemma 3.37, we can perturb the collection $\left(\varphi_{0}, \ldots, \varphi_{l}\right)$ into $\left(\tilde{\varphi}_{0}, \ldots, \tilde{\varphi}_{l}\right) \in \Sigma_{J}$, with $\tilde{\varphi}_{i}(y)=0$ and $\mathrm{d} \tilde{\varphi}_{i}(y)=v_{i}$. Thus, the polynomial $\sum_{i=0}^{l} \lambda_{i} \tilde{\varphi}_{i} \in \Sigma_{A_{J}}$ has an isolated line of singular points in its zero set, and $\Sigma_{A_{J}}=\Sigma_{J}$ is a hypersurface by Lemma 3.9.

(3) In the general case, in the same way as above, we can perturb the collection $\left(\varphi_{0}, \ldots, \varphi_{l}\right)$ into $\left(\tilde{\varphi}_{0}, \ldots, \tilde{\varphi}_{l}\right) \in \Sigma_{\{0, \ldots, l\}}$. But $\Sigma_{\{0, \ldots, l\}}$ is either a hypersurface itself, or is contained in a hypersurface $\widetilde{S}_{A_{0}^{\prime}, \ldots, A_{l}^{\prime}}$ (see Cases 1 and 2). 


\subsection{Proof of GKZ Decomposition Formula}

To deduce Gelfand-Kapranov-Zelevinsky's decomposition formula (Proposition 3.10) from the relative Kouchnirenko-Bernstein formula, we reformulate the definition of the $A$-determinant as follows (we use notation and facts from Sect. 2.6).

Geometric Characterization of A-resultant and A-determinant Denote the projection $(\mathbb{C} \backslash 0)^{k} \times \mathbb{C}\left[A_{0}\right] \oplus \cdots \oplus \mathbb{C}\left[A_{k}\right] \rightarrow \mathbb{C}\left[A_{0}\right] \oplus \cdots \oplus \mathbb{C}\left[A_{k}\right]$ by $p$. Let $R_{i}$ be the tautological polynomial on $(\mathbb{C} \backslash 0)^{k} \times \mathbb{C}\left[A_{0}\right] \oplus \cdots \oplus \mathbb{C}\left[A_{k}\right]$ that assigns the number $\varphi_{i}(y)$ to a point $\left(y, \varphi_{0}, \ldots, \varphi_{k}\right) \in(\mathbb{C} \backslash 0)^{k} \times \mathbb{C}\left[A_{0}\right] \oplus \cdots \oplus \mathbb{C}\left[A_{k}\right]$. Denote the convex hull of $A_{i}$ by $B_{i}$, and the dual fan of $B_{0}+\cdots+B_{k}$ by $\Sigma$. Then $s_{B_{i}} \cdot R_{i}$ extends to a section $\widetilde{R}_{i}$ of the line bundle $I_{B_{i}}$ on the product $\mathbb{T}^{\Sigma} \times \mathbb{C}\left[A_{0}\right] \oplus \cdots \oplus \mathbb{C}\left[A_{k}\right]$ (see Sect. 2.3 for the notation $I_{B}$ and $s_{B}$ ), and we can reformulate the definition of the resultant $R_{A_{0}, \ldots, A_{k}}$ as follows.

Lemma 3.42 $\left[R_{A_{0}, \ldots, A_{k}}=0\right]=p_{*}\left[\widetilde{R}_{0}=\cdots=\widetilde{R}_{k}=0\right]$.

We also need a similar description for the $A$-determinant. Denote the projection $(\mathbb{C} \backslash 0)^{k} \times \mathbb{C}[A] \rightarrow \mathbb{C}[A]$ by $p$. Let $S_{0}$ be the tautological polynomial on $(\mathbb{C} \backslash 0)^{k} \times$ $\mathbb{C}[A]$ that assigns the number $\varphi(y)$ to a point $(y, \varphi) \in(\mathbb{C} \backslash 0)^{k} \times \mathbb{C}[A]$, and let $S_{i}$ be $y_{i} \frac{\partial S_{0}}{\partial y_{i}}$, where $y_{1}, \ldots, y_{k}$ are the standard coordinates on $(\mathbb{C} \backslash 0)^{k}$. Denote the convex hull of $A$ by $B$ and its dual fan by $\Sigma$. Then $s_{B} \cdot S_{i}$ extends to a section $\widetilde{S}_{i}$ of the line bundle $I_{B}$ on the product $\mathbb{T}^{\Sigma} \times \mathbb{C}[A]$, and we can reformulate the definition of the $A$-determinant as follows.

Lemma 3.43 $\left[E_{A}=0\right]=p_{*}\left[\widetilde{S}_{0}=\cdots=\widetilde{S}_{k}=0\right]$.

Proof Let $j$ be the inclusion $\mathbb{C}[A] \hookrightarrow \underbrace{C[A] \oplus \cdots \oplus \mathbb{C}[A]}_{k+1}$ that assigns the collection $\left(\varphi, y_{1} \frac{\partial \varphi}{\partial y_{1}}, \ldots, y_{k} \frac{\partial \varphi}{\partial y_{k}}\right)$ to every $\varphi \in \mathbb{C}[A]$, and denote the induced inclusion $(\mathbb{C} \backslash 0)^{k} \times$ $\mathbb{C}[A] \hookrightarrow(\mathbb{C} \backslash 0)^{k} \times \underbrace{C[A] \oplus \cdots \oplus \mathbb{C}[A]}_{k+1}$ by the same letter $j$. Then

$$
\begin{aligned}
p_{*}\left[\widetilde{S}_{0}=\cdots=\widetilde{S}_{k}=0\right] & =p_{*} j^{*}\left[\widetilde{R}_{0}=\cdots=\widetilde{R}_{k}=0\right] \\
& =j^{*} p_{*}\left[\widetilde{R}_{0}=\cdots=\widetilde{R}_{k}=0\right] \\
& =j^{*}\left[R_{A_{0}, \ldots, A_{k}}=0\right]=\left[E_{A}=0\right],
\end{aligned}
$$

where the last two equalities are by Lemma 3.42 and by definition of the $A$ determinant respectively.

Proof of Proposition 3.10 In the notation of Lemma 3.43, represent the complete intersection $\left[\widetilde{S}_{0}=\cdots=\widetilde{S}_{k}=0\right]$ as a linear combination of irreducible varieties $\sum a_{i} V_{i}$. For every $V_{i}$, let $\mathbb{T}_{i}$ be the minimal orbit of the toric variety $\mathbb{T}^{\Sigma}$, such that $V_{i}$ is contained in the closure of $\mathbb{T}_{i} \times \mathbb{C}[A]$. For an arbitrary face $A^{\prime}$ of the set $A$, we denote the corresponding orbit of $\mathbb{T}^{\Sigma}$ by $\mathbb{T}_{A^{\prime}}$, and denote the sum $\sum_{i \mid \mathbb{T}_{i}=\mathbb{T}_{A^{\prime}}} a_{i} V_{i}$ by $V_{A^{\prime}}$. To 
prove the equality $\left[E_{A}=0\right]=\sum_{A^{\prime} \subset A} c^{A^{\prime}, A}\left[D_{A^{\prime}}=0\right]$ (which is exactly the statement of Proposition 3.10), it is enough to prove the following lemma.

Lemma $3.44 p_{*}\left(V_{A^{\prime}}\right)=c^{A^{\prime}, A}\left[D_{A^{\prime}}=0\right]$.

Proof Denote $\operatorname{dim} A^{\prime}$ by $l$, and choose a generic real $(k+1) \times(k+1)$-matrix $M$, whose first $l+1$ rows generate the vector span of the set $\{1\} \times A^{\prime} \subset \mathbb{Z} \oplus \mathbb{Z}^{k}$. Denote the entries of the product $M \cdot\left(\widetilde{S}_{0}, \ldots, \widetilde{S}_{k}\right)^{T}$ by $Z_{0}, \ldots, Z_{k}$ (recall that they are sections of the line bundle $I_{B}$ ). The desired equality is a corollary of the following facts:

(1) $\left[E_{A}=0\right]=p_{*}\left(\left[Z_{0}=\cdots=Z_{l-1}=0\right] \cap\left[Z_{l}=\cdots=Z_{k}=0\right]\right)$ by Lemma 3.43.

(2) The orbit $\mathbb{T}_{A^{\prime}}$ is a component of the complete intersection $\left[Z_{0}=\cdots=Z_{l-1}=0\right]$ of multiplicity $c^{A^{\prime}, A}$ by Lemma 2.28 .

(3) The complete intersection $\left[Z_{l}=\cdots=Z_{k}=0\right]$ intersects the orbit $\mathbb{T}_{A^{\prime}}$ transversally, and $p_{*}\left(\left[Z_{l}=\cdots=Z_{k}=0\right] \cap \mathbb{T}_{A^{\prime}}\right)=\left[D_{A^{\prime}}=0\right]$ by definition of the $A$ discriminant.

\section{Eliminants}

In the first subsection, we formulate a local version of elimination theory in the context of Newton polyhedra (the global version is presented in [13]); i.e., we study the Newton polyhedron and leading coefficients of the equation of a projection of a complete intersection which is defined by equations with given Newton polyhedra and generic leading coefficients. The main result is Theorem 4.3; the proof is given in Sect. 4.2. In Sect. 4.3, we specialize this to $A$-determinants.

\subsection{Elimination Theory}

Notation Let $\tau \subsetneq\left(\mathbb{R}^{n}\right)^{*}$ be a convex $n$-dimensional rational polyhedral strictly convex cone (i.e., a cone that does not contain a line), denote its dual cone $\{v \in$ $\mathbb{R}^{n} \mid \gamma(v) \geqslant 0$ for $\left.\gamma \in \tau\right\}$ by $\tau^{\vee}$, and consider the corresponding affine toric variety $\mathbb{T}^{\tau}=\operatorname{spec} \mathbb{C}\left[\tau^{\vee}\right]$ with the maximal torus $(\mathbb{C} \backslash 0)^{n}$ and the vertex $O$. Note that $\tau^{\vee}$ is unbounded. A germ of a meromorphic function on $\mathbb{T}^{\tau}$ near $O$ with no poles in the maximal torus can be represented as a power series $f(x)=\sum_{b \in \mathbb{Z}^{n}} c_{b} x^{b}$ for $x \in(\mathbb{C} \backslash 0)^{n}$, and the convex hull of the set $\left\{b \mid c_{b} \neq 0\right\}+\tau^{\vee}$ is called the Newton polyhedron $\Delta_{f}$ of $f$. The union of all bounded faces of $\Delta_{f}$ is called the Newton diagram $\partial \Delta_{f}$, and the coefficients $c_{b}, b \in \partial \Delta_{f}$, are called the leading coefficients of the germ $f$.

Eliminant Let $A_{0}, \ldots, A_{k}$ be finite sets in $\mathbb{Z}^{k}$. For $i=0, \ldots, k$ and $a \in A_{i}$, let $f_{a, i}$ be a germ of a meromorphic function on the toric variety $\mathbb{T}^{\tau}$ with no poles in the maximal torus. We define the germs of functions $F_{0}, \ldots, F_{k}$ on $\mathbb{T}^{\tau} \times(\mathbb{C} \backslash 0)^{k}$ by the formula

$$
F_{i}(x, y)=\sum_{a \in A_{i}} f_{a, i}(x) y^{a} \quad \text { for } x \in \mathbb{T}^{\tau}, y \in(\mathbb{C} \backslash 0)^{k},
$$


note that $F_{i}(x, \cdot) \in \mathbb{C}\left[A_{i}\right]$, and denote the number $R_{A_{0}, \ldots, A_{k}}\left(F_{0}(x, \cdot), \ldots, F_{k}(x, \cdot)\right)$ by $R_{F_{0}, \ldots, F_{k}}(x)$ (see Sect. 3.1 for the definition of the resultant $R_{A_{0}, \ldots, A_{k}}$ ).

Definition 4.1 The function $R_{F_{0}, \ldots, F_{k}}$ on the toric variety $\mathbb{T}^{\tau}$ is called the eliminant of the projection of the complete intersection $F_{0}=\cdots=F_{k}=0$ to $\mathbb{T}^{\tau}$.

The geometric meaning of the function $R_{F_{0}, \ldots, F_{k}}$ is as follows: if leading coefficients of the functions $f_{a, i}$ are in general position, and the image of the complete intersection $F_{0}=\cdots=F_{k}=0$ under the projection $\mathbb{T}^{\tau} \times(\mathbb{C} \backslash 0)^{k} \rightarrow \mathbb{T}^{\tau}$ has codimension 1 , then the closure of this image is the zero locus of $R_{F_{0}, \ldots, F_{k}}$ (see the beginning of the next subsection for details). Under these assumptions, the Newton polyhedron of $R_{F_{0}, \ldots, F_{k}}$ does not depend on coefficients of the functions $f_{a, i}$, but only on their Newton polyhedra. Theorem 4.3 below solves the following problem:

Express the Newton polyhedron and leading coefficients of the eliminant $R_{F_{0}, \ldots, F_{k}}$ in terms of the Newton polyhedra and leading coefficients of the functions $f_{a, i}$, provided that leading coefficients are in general position, and describe this condition of general position explicitly.

Condition of General Position We denote the Newton polyhedron of $f_{a, i}$ by $\Delta_{a, i}$, and define the Newton polyhedron $\Delta_{i}$ of the function $F_{i}$ as the convex hull of the set $\bigcup_{a \in A_{i}} \Delta_{a, i} \times\{a\} \subset \mathbb{R}^{n} \oplus \mathbb{R}^{k}$; then $F_{i}(z)$ can be represented as a power series $\sum_{b \in \Delta_{i}} c_{b, i} z^{b}$ for $z \in(\mathbb{C} \backslash 0)^{n} \times(\mathbb{C} \backslash 0)^{k}$. If $\Gamma$ is a face of $\Delta_{i}$, then we denote the function $\sum_{b \in \Gamma} c_{b, i} z^{b}$ by $F_{i}^{\Gamma}$.

Definition 4.2 The leading coefficients of the functions $F_{0}, \ldots, F_{k}$ are said to be in general position if, for every collection of compatible bounded faces $\Gamma_{i} \subset \Delta_{i}$, $i=0, \ldots, k$ (see Definition 2.13), such that the restriction of the projection $\mathbb{R}^{n} \oplus$ $\mathbb{R}^{k} \rightarrow \mathbb{R}^{k}$ to $\Gamma_{0}+\cdots+\Gamma_{k}$ is injective, the system of polynomial equations $F_{0}^{\Gamma_{0}}=$ $\cdots=F_{k}^{\Gamma_{k}}=0$ has no solutions in $(\mathbb{C} \backslash 0)^{n} \times(\mathbb{C} \backslash 0)^{k}$.

This condition is obviously satisfied for generic leading coefficients of the functions $f_{a, i}$. Note that this condition is slightly weaker than the one in [13]. For example, if a face $\Gamma_{i} \subset \Delta_{i}$ is contained in a fiber of the projection $\mathbb{R}^{n} \oplus \mathbb{R}^{k} \rightarrow \mathbb{R}^{k}$, then we do not impose any assumptions on the leading coefficients of $F_{i}$, corresponding to internal integer points of $\Gamma_{i}$. This slight difference is important for our purpose (cf. [15]); see the proof of Proposition 4.7 below.

Elimination Theorem We recall that the Minkowski integral $\int \Delta \subset \mathbb{R}^{n}$ of a polyhedron $\Delta \subset \mathbb{R}^{n} \oplus \mathbb{R}^{k}$ is defined in Sect. 1. For bounded faces $\Gamma_{i} \subset \Delta_{i}, i=0, \ldots, k$, we denote the intersection of $A_{i}$ with the image of $\Gamma_{i}$ under the projection $\mathbb{R}^{n} \oplus \mathbb{R}^{k} \rightarrow$ $\mathbb{R}^{k}$ by $\widetilde{\Gamma}_{i}$, and the value $R_{\widetilde{\Gamma}_{0}, \ldots, \widetilde{\Gamma}_{k}}\left(F_{0}^{\Gamma_{0}}(x, \cdot), \ldots, F_{k}^{\Gamma_{k}}(x, \cdot)\right)$ by $R_{F_{0} \Gamma_{0}, \ldots, F_{k}}(x)$ for $x \in(\mathbb{C} \backslash 0)^{n}$. Then the Laurent polynomial $R_{F_{0} \Gamma_{0}, \ldots, F_{k} \Gamma_{k}}$ on $(\mathbb{C} \backslash 0)^{n}$ depends only on leading coefficients of the functions $f_{a, i}$. 


\section{Theorem 4.3}

(1) The Newton polyhedron of the eliminant $R_{F_{0}, \ldots, F_{k}}$ is contained in the mixed fiber polyhedron

$$
\operatorname{MP}\left(\Delta_{0}, \ldots, \Delta_{k}\right) .
$$

These two polyhedra coincide if and only if the leading coefficients of $F_{0}, \ldots, F_{k}$ are in general position in the sense of Definition 4.2.

(2) For every face $\Gamma$ of the polyhedron $\operatorname{MP}\left(\Delta_{0}, \ldots, \Delta_{k}\right) \subset \mathbb{R}^{n} \subset \mathbb{R}^{n} \oplus \mathbb{R}^{k}$,

$$
R_{F_{0}, \ldots, F_{k}}^{\Gamma_{0}}=\prod_{\Gamma_{0}, \ldots, \Gamma_{k}} R_{F_{0} \Gamma_{0}, \ldots, F_{k} \Gamma_{k}},
$$

where the collection $\left(\Gamma_{0}, \ldots, \Gamma_{k}\right)$ runs over all collections of faces of the polyhedra $\Delta_{0}, \ldots, \Delta_{k}$, such that $\Gamma, \Gamma_{0}, \ldots, \Gamma_{k}$ are compatible.

The proof of a global version of this theorem is given in [13], but cannot be extended to the local case word by word.

\subsection{Proof of Theorem 4.3}

Geometric Characterization of Eliminant We can describe the geometric meaning of the eliminant $R_{F_{0}, \ldots, F_{k}}$ as follows (we assume that all functions $f_{a, i}, a \in A_{i}$, are holomorphic for simplicity). Denote the convex hull of $A_{i}$ by $B_{i}$, and the dual fan of $B_{0}+\cdots+B_{k}$ by $\Sigma$. Then the product $s_{B_{i}} \cdot F_{i}$ extends to a section $\widetilde{F}_{i}$ of the line bundle $I_{B_{i}}$ on the product $\mathbb{T}^{\Sigma} \times \mathbb{T}^{\tau}$ (we use the notation $I_{B}, s_{B}$, and $m\left(f_{1} \cdots f_{k} \cdot V\right.$ ) introduced in Sect. 2.3). Denote the projection $\mathbb{T}^{\Sigma} \times \mathbb{T}^{\tau} \rightarrow \mathbb{T}^{\tau}$ by $p$.

Lemma 4.4 For a germ of a curve $C \subset \mathbb{T}^{\tau}$ near the origin,

$$
m\left(R_{F_{0}, \ldots, F_{k}} \cdot C\right)=m\left(\widetilde{F}_{0} \ldots \widetilde{F}_{k} \cdot p^{(-1)}(C)\right) .
$$

In particular, both parts of the equality are well defined simultaneously.

Proof We first consider the special case $R_{F_{0}, \ldots, F_{k}}=R_{A_{0}, \ldots, A_{k}}$. In this case $\mathbb{T}^{\tau}=$ $\mathbb{C}\left[A_{0}\right] \oplus \cdots \oplus \mathbb{C}\left[A_{k}\right]$, and the function $F_{i}$ equals the tautological function $R_{i}$ on $(\mathbb{C} \backslash 0)^{k} \times \mathbb{C}\left[A_{0}\right] \oplus \cdots \oplus \mathbb{C}\left[A_{k}\right]$, that maps a point $\left(x, \varphi_{0}, \ldots, \varphi_{k}\right)$ to $\varphi_{i}(x)$. Accordingly, we denote the section $\widetilde{F}_{i}$ by $\widetilde{R}_{i}$ in this case.

(1) If $R_{F_{0}, \ldots, F_{k}}=R_{A_{0}, \ldots, A_{k}}$, and $C$ intersects the set $R_{A_{0}, \ldots, A_{k}}=0$ transversally, then the statement follows by definition of the $A$-resultant (Definition 3.1).

(2) If $R_{F_{0}, \ldots, F_{k}}=R_{A_{0}, \ldots, A_{k}}$, and $C$ intersects the set $R_{A_{0}, \ldots, A_{k}}=0$ properly, then we can perturb $C$ so that it intersects the set $R_{A_{0}, \ldots, A_{k}}=0$ transversally at a finite number of points, which reduces the statement to the case (1).

(3) In general, consider the map $\mathcal{F}: \mathbb{T}^{\tau} \rightarrow \mathbb{C}\left[A_{0}\right] \oplus \cdots \oplus \mathbb{C}\left[A_{k}\right]$ that assigns the collection of polynomials $\left(F_{0}(x, \cdot), \ldots, F_{k}(x, \cdot)\right)$ to every point $x \in \mathbb{T}^{\tau}$. Accordingly, denote the induced map $\mathbb{T}^{\Sigma} \times \mathbb{T}^{\tau} \rightarrow \mathbb{T}^{\Sigma} \times \mathbb{C}\left[A_{0}\right] \oplus \cdots \oplus \mathbb{C}\left[A_{k}\right]$ by (id, $\left.\mathcal{F}\right)$. Then we have

$$
R_{F_{0}, \ldots, F_{k}}=R_{A_{0}, \ldots, A_{k}} \circ \mathcal{F} \quad \text { and } \quad \widetilde{F}_{i}=(\mathrm{id}, \mathcal{F})^{*} \widetilde{R}_{i},
$$

which reduces the general case to the case (2). 
Proof of Theorem 4.3 First, we can assume without loss of generality that all germs $f_{a, i}, a \in A_{i}$, are holomorphic. Indeed, if we multiply every $f_{a, i}$ by a monomial $x^{c_{i}}$, where $c_{i} \in \tau^{\vee}$ is far enough from the boundary of $\tau^{\vee}$, then all functions $f_{a, i}$ become holomorphic, while the statement of Theorem 4.3 does not change because of the homogeneity of the $\left(A_{0}, \ldots, A_{k}\right)$-resultant.

Proof of Part 1 For an arbitrary positive integer linear function $l$ on the cone $\tau^{\vee}$, let $\left(l_{1}, \ldots, l_{n}\right)$ be its differential, pick generic complex numbers $c_{1}, \ldots, c_{n}$, and consider the corresponding germ of a monomial curve $C: \mathbb{C} \rightarrow \mathbb{T}^{\tau \vee}$ defined by the formula $C(t)=\left(c_{1} t^{l_{1}}, \ldots, c_{n} t^{l_{n}}\right) \in(\mathbb{C} \backslash 0)^{n}$ for $t \neq 0$. Then the minimal value of $l$ on the Newton polyhedron of the eliminant $R_{F_{0}, \ldots, F_{k}}$ equals the intersection number $m\left(R_{F_{0}, \ldots, F_{k}} \cdot C\right)$, which, by Lemma 4.4 , equals

$$
m\left(\widetilde{F}_{0} \cdots \widetilde{F}_{k} \cdot p^{(-1)}(C)\right) .
$$

Denote the convex hull of $A_{i}$ by $B_{i}$, the projection $\mathbb{R}^{k} \oplus \mathbb{R}^{n} \rightarrow \mathbb{R}^{k} \oplus \mathbb{R}$ along $\operatorname{ker} l \subset \mathbb{R}^{n}$ by $\pi_{l}$, and the restriction of the germ $F_{i}$ to the toric variety $p^{(-1)}(C)$ by $G_{i}$. If the leading coefficients of the functions $F_{0}, \ldots, F_{k}$ are in general position in the sense of Definition 4.2, and the exponents $l_{1}, \ldots, l_{n}$ are generic in the sense that the restriction of the projection $\pi_{l}$ to $\Delta_{0}+\cdots+\Delta_{k}$ is one to one over bounded faces of its image, then the Newton polyhedron of $G_{i}$ equals $\pi_{l}\left(\Delta_{i}\right)$, and the leading coefficients of $G_{i}$ are in general position in the sense of Definition 2.14. Thus, by Theorem 2.15, we have

$m\left(\widetilde{F}_{0} \ldots \widetilde{F}_{k} \cdot p^{(-1)}(C)\right)=(k+1) ! \operatorname{MV}\left(\left(\pi_{l} \Delta_{0}, B_{0} \times \mathbb{R}_{+}\right), \ldots,\left(\pi_{l} \Delta_{k}, B_{k} \times \mathbb{R}_{+}\right)\right)$.

Thus, if $\mathcal{N}$ is the desired Newton polyhedron of the eliminant $R_{F_{0}, \ldots, F_{k}}$, then, for every positive integer linear function $l$ on the cone $\tau^{\vee}$, we have

$$
\left.\min l\right|_{\mathcal{N}}=(k+1) ! \operatorname{MV}\left(\left(\pi_{l} \Delta_{0}, B_{0} \times \mathbb{R}_{+}\right), \ldots,\left(\pi_{l} \Delta_{k}, B_{0} \times \mathbb{R}_{+}\right)\right) .
$$

This is exactly the formula for the support function of the mixed fiber polyhedron $\operatorname{MP}\left(\Delta_{0}, \ldots, \Delta_{k}\right)$; see Proposition 2.10.

Remark Suppose that, on the contrary, the condition of general position of Definition 4.2 is not satisfied for some compatible faces $\Gamma_{0}, \ldots, \Gamma_{k}$ of the polyhedra $\Delta_{0}, \ldots, \Delta_{k}$. Then the Newton polyhedron of the eliminant $R_{F_{0}, \ldots, F_{k}}$ is strictly smaller than the mixed fiber polyhedron $\operatorname{MP}\left(\Delta_{0}, \ldots, \Delta_{k}\right)$.

Namely, pick the face $\Gamma$ of the polyhedron $\operatorname{MP}\left(\Delta_{0}, \ldots, \Delta_{k}\right)$, compatible with $\Gamma_{0}, \ldots, \Gamma_{k}$. Consider a linear function $l$ that attains its minimum on $\Gamma$ as a function on $\operatorname{MP}\left(\Delta_{0}, \ldots, \Delta_{k}\right)$. Then, in the notation of the proof of Part 1 , the leading coefficients of the functions $G_{0}, \ldots, G_{k}$ are not in general position in the sense of Definition 2.14; thus,

$$
\left.\min l\right|_{\mathcal{N}}>(k+1) ! \operatorname{MV}\left(\left(\pi_{l} \Delta_{0}, B_{0} \times \mathbb{R}_{+}\right), \ldots,\left(\pi_{l} \Delta_{k}, B_{0} \times \mathbb{R}_{+}\right)\right),
$$

and thus, the face $\Gamma$ is not contained in the Newton polyhedron of the eliminant $R_{F_{0}, \ldots, F_{k}}$. 
Proof of Part 2 First, suppose that the condition of general position of Definition 4.2 is not satisfied for some faces $\Gamma_{0}, \ldots, \Gamma_{k}$ of the polyhedra $\Delta_{0}, \ldots, \Delta_{k}$, compatible with the face $\Gamma$. Then the corresponding multiplier on the right-hand side of the desired equality vanishes. On the other hand, by the remark above, the left-hand side vanishes as well.

Suppose that, on the contrary, the condition of general position of Definition 4.2 is satisfied for all collections of faces $\Gamma_{0}, \ldots, \Gamma_{k}$ of the polyhedra $\Delta_{0}, \ldots, \Delta_{k}$, compatible with the face $\Gamma$. Then the desired equality is proved in [13]. Note that the proof in [13] is written in the global setting, with a complex torus instead of the toric variety $\mathbb{T}^{\tau}$, and polynomials instead of analytic functions on it. However, one can readily verify that the same proof remains valid in the local setting as well.

\subsection{Reach Discriminants and Their Newton Polyhedra}

The following version of the discriminant of a projection is not what we promised to study in the introduction; nevertheless, it allows us to reduce the study of discriminants of projections to elimination theory. Let $A$ be a finite set in $\mathbb{Z}^{k}$, and let $f_{a}$ be a germ of a meromorphic function on the toric variety $\mathbb{T}^{\tau}$ for every $a \in A$. Define the germ of a function $F$ on $\mathbb{T}^{\tau} \times(\mathbb{C} \backslash 0)^{k}$ by the formula

$$
F(x, y)=\sum_{a \in A} f_{a}(x) y^{a} \quad \text { for } x \in \mathbb{T}^{\tau}, y \in(\mathbb{C} \backslash 0)^{k},
$$

and denote the number $E_{A}(F(x, \cdot))$ by $E_{F}(x)$ for every $x \in \mathbb{T}^{\tau}$ near the origin (see Sect. 3.1 for the definition of the $A$-determinant $E_{A}$ ).

Definition 4.5 The function $E_{F}$ on the toric variety $\mathbb{T}^{\tau}$ is called the reach discriminant of the projection of the hypersurface $F=0$ to $\mathbb{T}^{\tau}$.

We denote the Newton polyhedron of $f_{a}$ by $\Delta_{a}$, and define the Newton polyhedron $\Delta$ of the function $F$ as the convex hull of the set $\bigcup_{a \in A} \Delta_{a} \times\{a\} \subset \mathbb{R}^{n} \oplus \mathbb{R}^{k}$; then $F(z)$ can be represented as a power series $\sum_{b \in \Delta} c_{b} z^{b}$ for $z \in(\mathbb{C} \backslash 0)^{n} \times(\mathbb{C} \backslash 0)^{k}$. For any $\Gamma \subset \mathbb{R}^{n} \oplus \mathbb{R}^{k}$, we denote the function $\sum_{b \in \Gamma} c_{b} z^{b}$ by $F^{\Gamma}$.

Definition 4.6 The leading coefficients of the functions $f_{a}, a \in A$, are said to be in general position if, for every bounded face $\Gamma \subset \Delta$, such that the restriction of the projection $\mathbb{R}^{n} \oplus \mathbb{R}^{k} \rightarrow \mathbb{R}^{k}$ to $\Gamma$ is injective, 0 is a regular value of the Laurent polynomial $F^{\Gamma}$.

Obviously, this condition is satisfied for generic leading coefficients of the functions $f_{a}$.

\section{Proposition 4.7}

(1) If the leading coefficients of the functions $f_{a}, a \in A$ are in general position in the sense of Definition 4.6, then the Newton polyhedron of $E_{F}$ equals $\int \Delta$. 
(2) For every bounded face $\Gamma$ of the polyhedron $\int \Delta \subset \mathbb{R}^{n} \subset \mathbb{R}^{n} \oplus \mathbb{R}^{k}$,

$$
E_{F}^{\Gamma}=\prod E_{F^{\Gamma^{\prime}}},
$$

where $\Gamma^{\prime}$ runs over all compatible with $\Gamma$ bounded faces $\Gamma^{\prime}$ of the polyhedron $\Delta \subset \mathbb{R}^{n} \oplus \mathbb{R}^{k}$, such that the image of $\Gamma^{\prime}$ under the projection $\mathbb{R}^{n} \oplus \mathbb{R}^{k} \rightarrow \mathbb{R}^{k}$ has the same dimension as $A$.

Proof We can assume that $\operatorname{dim} A=k$ without loss of generality (otherwise, we can dehomogenize the function $F$ ). Consider $k+1$ generic linear combinations of the functions $F, y_{1} \frac{\partial F}{\partial y_{1}}, \ldots, y_{k} \frac{\partial F}{\partial y_{k}}$, where $y_{1}, \ldots, y_{k}$ are the standard coordinates on the torus $(\mathbb{C} \backslash 0)^{k}$. We denote these linear combinations by $F_{0}, \ldots, F_{k}$, and note that $\Delta$ is the Newton polyhedron of each of these functions (while it is not always the Newton polyhedron of the functions $y_{i} \frac{\partial F}{\partial y_{i}}$ ). General position for the leading coefficients of the functions $f_{a}, a \in A$, in the sense of Definition 4.6 implies general position for the leading coefficients of the functions $F_{0}, \ldots, F_{k}$ in the sense of Definition 4.2. Thus, the statement of Proposition 4.7 follows from Theorem 4.3 for the functions $F_{0}, \ldots, F_{k}$.

\section{Discriminants of Hypersurfaces}

In this section, we study the Newton polyhedron and leading coefficients of the discriminant of a projection of an analytic hypersurface, whose Newton polyhedron is given and whose leading coefficients are in general position.

In the first subsection, we give an "algebraic" definition of the discriminant, and clarify its geometric meaning in Propositions 5.2 and 5.3; the proof of these facts occupies Sects. 5.2 and 5.3. In Sect. 5.4, we study the Newton polyhedron (Theorem 5.10) and leading coefficients (Proposition 5.11) of the discriminant. These results are proved in the last subsection.

\subsection{Discriminants of Hypersurfaces}

Let $A$ be a finite set in $\mathbb{Z}^{k}$, let $\tau \subset\left(\mathbb{R}^{n}\right)^{*}$ be a convex $n$-dimensional rational polyhedral cone that does not contain a line, and let $f_{a}$ be a germ of a meromorphic function on the affine toric variety $\mathbb{T}^{\tau}$ for every $a \in A$. Define the germ of a function $F$ on $\mathbb{T}^{\tau} \times(\mathbb{C} \backslash 0)^{k}$ by the formula

$$
F(x, y)=\sum_{a \in A} f_{a}(x) y^{a} \quad \text { for } x \in \mathbb{T}^{\tau}, y \in(\mathbb{C} \backslash 0)^{k},
$$

note that $F(x, \cdot) \in \mathbb{C}[A]$, and denote the number $D_{A}(F(x, \cdot))$ by $D_{F}(x)$ (see Sect. 3.2 for the definition of the $A$-discriminant $D_{A}$ ).

Definition 5.1 The function $D_{F}$ on the toric variety $\mathbb{T}^{\tau}$ is called the discriminant of the projection of the hypersurface $F=0$ to $\mathbb{T}^{\tau}$. 
The discriminant has the expected geometric meaning if the leading coefficients are in general position. Namely, denote the set $\left\{x \in(\mathbb{C} \backslash 0)^{n} \mid D_{F}(x)=0\right\}$ by $Z(F)$, and consider the set $\Sigma(F)$ of all $x \in(\mathbb{C} \backslash 0)^{n}$ such that 0 is a singular value of the polynomial $F(x, \cdot)$ on $(\mathbb{C} \backslash 0)^{k}$.

Proposition 5.2 Suppose that the Newton polyhedra of the functions $f_{a}, a \in A$, are given, and the leading coefficients of these functions are in general position. If $A$ is not dual defect (for example, if A satisfies assumptions of Proposition 3.14), then $\overline{\Sigma(F)}=\overline{Z(F)}$ in $(\mathbb{C} \backslash 0)^{n}$, otherwise codim $\Sigma(F)>1$.

This statement can be extended from the maximal torus $(\mathbb{C} \backslash 0)^{n}$ to the toric variety $\mathbb{T}^{\tau}$ as follows. For a face $\theta$ of the cone $\tau^{\vee} \subset \mathbb{R}^{n}$, define $A(\theta)$ as the set of all $a$ such that the Newton polyhedron of $f_{a}$ intersects $\theta$. Consider the set $\Sigma_{0}(F)$ of all $x \in \mathbb{T}^{\tau}$ such that 0 is a singular value of the polynomial $F(x, \cdot)$ on $(\mathbb{C} \backslash 0)^{k}$.

Proposition 5.3 Suppose that the functions $f_{a}, a \in A$, are holomorphic, their Newton polyhedra are such that $A(\theta) \neq \varnothing$ for every codimension 1 face $\theta \subset \tau^{\vee}$, and their leading coefficients are in general position. Then

(1) the union of all codimension 1 components of $\overline{\Sigma_{0}(F)}$ equals $\overline{Z(F)}$ in $\mathbb{T}^{\tau}$.

(2) If, in addition, $A$ is not dual defect and $\operatorname{dim} A(\theta)>\operatorname{dim} A+\operatorname{dim} \theta-n$ for every $\theta \neq \tau^{\vee}$, then $\overline{\Sigma_{0}(F)}=\overline{Z(F)}$ (in particular, $\overline{\Sigma_{0}(F)}$ is a hypersurface).

Note that $\overline{Z(F)}$ is contained in the zero set of the discriminant $D_{F}$ on $\mathbb{T}^{\tau}$, but is smaller in general (even under the assumptions of the proposition). The equality of Proposition 5.3(2) may turn into the strict inequality $\overline{\Sigma_{0}(F)} \varsubsetneqq \overline{Z(F)}$ in the case of arbitrary leading coefficients of the functions $f_{a}$, and even this inequality may not be valid if $\operatorname{dim} A(\theta)<\operatorname{dim} A+\operatorname{dim} \theta-n$ for some $\theta$. One can readily observe corresponding examples in the simplest nontrivial case $n=1, A=\{0,1,2\} \subset \mathbb{Z}^{1}$; a more refined example with $\operatorname{dim} A(\theta)=\operatorname{dim} A+\operatorname{dim} \theta-n$ and $\Sigma(F)$ not of pure dimension is given at the end of Sect. 5.3.

\subsection{Maps with Generic Leading Coefficients}

To prove the statements above, we need the following.

Proposition 5.4 Let $h_{1}, \ldots, h_{p}$ be either

(1) Laurent polynomials on the complex torus $(\mathbb{C} \backslash 0)^{n}$, or

(2) germs of meromorphic functions on an affine toric variety $\left(\mathbb{T}^{\tau}, O\right)$ with no poles in the maximal torus $(\mathbb{C} \backslash 0)^{n}$.

In both cases, consider the map $h=\left(h_{1}, \ldots, h_{p}\right):(\mathbb{C} \backslash 0)^{n} \rightarrow \mathbb{C}^{p}$.

If $S \subset \mathbb{C}^{p}$ is an arbitrary algebraic set of codimension $s$, the Newton polyhedra of the functions $h_{i}$ are given, and the leading coefficients of these functions are in general position, then the set $h^{(-1)}(S)$ has the same codimension $s$. 
Note that, in both settings, $h$ is defined as a map from the torus $(\mathbb{C} \backslash 0)^{n}$, rather than from the toric variety $\left(\mathbb{T}^{\tau}, O\right)$, and, in particular, $h^{(-1)}(S) \subset(\mathbb{C} \backslash 0)^{n}$. If $S^{\prime} \subset$ $\mathbb{C}^{p}$ is a constructible set (i.e., if it is obtained by applying the operations of union, intersection, and subtraction to algebraic sets), then, applying Proposition 5.4 to its closure $\overline{S^{\prime}}$ and to the closure of the difference $\overline{S^{\prime}} \backslash S^{\prime}$, one gets the following.

Corollary 5.5 If $S^{\prime} \subset \mathbb{C}^{p}$ is a constructible set, then, under the assumptions of Proposition 5.4, the closure of $h^{(-1)}\left(S^{\prime}\right)$ equals $h^{(-1)}\left(\overline{S^{\prime}}\right)$.

To prove Proposition 5.4, we reduce it to the following lemma.

Lemma 5.6 Let $g_{1}, \ldots, g_{p}$ be Laurent polynomials on $(\mathbb{C} \backslash 0)^{k}$, whose coefficients are germs of meromorphic functions on an affine toric variety $\left(\mathbb{T}^{\tau}, O\right)$ with no poles in its maximal torus $(\mathbb{C} \backslash 0)^{n}$. If $Q \subset(\mathbb{C} \backslash 0)^{n} \times(\mathbb{C} \backslash 0)^{k}$ is an arbitrary algebraic set, the Newton polyhedra of the functions $g_{i}$ are given, and the leading coefficients of these functions are in general position, then the set $\left\{g_{1}=\cdots=g_{p}=0\right\}$ intersects $Q$ properly near $\{O\} \times(\mathbb{C} \backslash 0)^{k} \subset \mathbb{T}^{\tau} \times(\mathbb{C} \backslash 0)^{k}$.

Definition 5.7 For a covector $\gamma \in\left(\mathbb{Z}^{n}\right)^{*}$ and an analytic function $g(y)=\sum_{a \in \mathbb{Z}^{n}} c_{a} y^{a}$ on $(\mathbb{C} \backslash 0)^{n}$, the $\gamma$-truncation $g^{\gamma}$ is defined to be the last nonzero sum in the sequence of sums $\sum_{a \mid \gamma(a)=k} c_{a} y^{a}, k \in \mathbb{Z}$, provided that these sums are equal to 0 for large $k$.

For a covector $\gamma \in\left(\mathbb{Z}^{n}\right)^{*}$ and an ideal $I$ in $\mathbb{C}\left[\mathbb{Z}^{n}\right]$, the $\gamma$-truncation $I^{\gamma}$ is the ideal, generated by $\gamma$-truncations of all elements of $I$. The $\gamma$-truncation $Q^{\gamma}$ of an algebraic variety $Q \in(\mathbb{C} \backslash 0)^{n}$ is defined to be the zero locus of the $\gamma$-truncation of its ideal.

Proof of Lemma 5.6 Choose any covector $\gamma \in\left(\mathbb{R}^{n} \times \mathbb{R}^{k}\right)^{*}$ that takes only negative values on the closed cone $\tau^{\vee} \times\{0\} \subset \mathbb{R}^{n} \times \mathbb{R}^{k}$. By the Bertini-Sard theorem, the set $\left\{g_{1}^{\gamma}=\cdots=g_{p}^{\gamma}=0\right\}$ intersects $Q^{\gamma}$ properly under an appropriate assumption of general position for the leading coefficients of the functions $g_{1}, \ldots, g_{p}$. Since the set of all possible varieties of the form $Q^{\gamma}$ is finite (see, e.g., [18], [28]), one can choose the latter assumption of general position to be independent of $\gamma$. Under this assumption, the set $\left\{g_{1}^{\gamma}=\cdots=g_{p}^{\gamma}=0\right\}$ intersects $Q^{\gamma}$ properly for every $\gamma$; thus, the same holds for $\left\{g_{1}=\cdots=g_{p}=0\right\}$ and $Q$ near the set $\{O\} \times(\mathbb{C} \backslash 0)^{k} \subset \mathbb{T}^{\tau} \times(\mathbb{C} \backslash 0)^{k}$ (see, e.g., [18], [28]).

In the same way, if $Q$ is smooth, one can prove that $\left\{g_{1}=\cdots=g_{p}=0\right\}$ intersects $Q$ transversally, if leading coefficients are in general position.

Proof of Proposition 5.4 Denote the standard coordinates on $\mathbb{C}^{p}$ by $y_{1}, \ldots, y_{p}$, consider an arbitrary subdivision $\{1, \ldots, p\}=I \sqcup J$, and denote the torus $\left\{\left(y_{1}, \ldots, y_{p}\right) \mid\right.$ $y_{i} \neq 0$ for $i \in I$ and $y_{j}=0$ for $\left.j \in J\right\}$ by $(\mathbb{C} \backslash 0)^{I}$, and apply Lemma 5.6 to the set $Q=\left(S \cap(\mathbb{C} \backslash 0)^{I}\right) \times \mathbb{T}^{\tau}$ and functions $g_{i}=\left\{\begin{array}{ll}h_{i}-y_{i} & \text { for } i \in I \\ h_{i} & \text { for } i \in J\end{array}\right\}$ on the toric variety $(\mathbb{C} \backslash 0)^{I} \times \mathbb{T}^{\tau}$. 


\subsection{Proofs of Propositions 5.2 and 5.3}

Proof of Proposition 5.2 Recall that $\Sigma \subset \mathbb{C}[A]$ is the set of all $\varphi$ such that $\varphi(y)=$ $\mathrm{d} \varphi(y)=0$ for some $y \in(\mathbb{C} \backslash 0)^{k}$. Its closure $\bar{\Sigma}$ is a hypersurface and is defined by the equation $D_{A}=0$ (otherwise, identically $D_{A}=1$ and $Z(F)=\varnothing$ by definition).

Let $\mathcal{F}: \mathbb{T}^{\tau} \rightarrow \mathbb{C}[A]$ be the map that assigns the polynomial $F(x, \cdot)$ to a point $x \in \mathbb{T}^{\tau}$. We can express the desired sets $\Sigma(F)$ and $Z(F)=\left\{D_{F}=0\right\}$ in terms of this map:

$$
\begin{gathered}
\Sigma(F)=\mathcal{F}^{(-1)}(\Sigma) \cap(\mathbb{C} \backslash 0)^{n}, \\
D_{F}=D_{A} \circ \mathcal{F} .
\end{gathered}
$$

Proposition 5.2 now follows by Corollary 5.4 with $\left(h_{1}, \ldots, h_{p}\right)=\mathcal{F}$ and $S^{\prime}=\Sigma$.

We can also formulate a refinement of Proposition 5.2, taking multiplicities into account (the proof follows the same lines but requires more technical details; we omit it, since we do not need this refinement in what follows). Restrict the projection $p: \mathbb{T}^{\tau} \times(\mathbb{C} \backslash 0)^{k} \rightarrow \mathbb{T}^{\tau}$ to the regular locus of the set $\{F=0\}$ and denote the singular locus of this restriction by $S$.

Proposition 5.8 (See Sect. 2.6 for the notation) $p_{*}(S)$ equals $\left[D_{\bar{F}}=0\right]$ on the complex torus $(\mathbb{C} \backslash 0)^{n}$, and does not contain codimension 1 orbits of the toric variety $\mathbb{T}^{\tau}$, if the leading coefficients of the functions $f_{a}, a \in A$, are in general position.

Proof of Proposition 5.3 For a closed regular subvariety $M \subset(\mathbb{C} \backslash 0)^{k}$, define $\Sigma(M) \subset \mathbb{C}[A]$ as the set of all Laurent polynomials $\varphi$ in $\mathbb{C}[A]$, such that the set $\{y \in M \mid \varphi(y)=\mathrm{d} \varphi(y)=0\}$ has at least one isolated point. This definition implies the following properties of $\Sigma(M)$ (in contrast to $\Sigma$ ):

(1) for every $\varphi \in \Sigma(M)$, at least one of the local components of $\Sigma(M)$ near $\varphi$ is closed.

(2) The set $\Sigma(M)$ has codimension $1+\operatorname{codim} M+\operatorname{dim} A-k$ at all of its points. We need the following corollary of (1) and (2):

(3) The set $\mathcal{F}^{(-1)}(\Sigma(M))$ has codimension at most $1+\operatorname{codim} M+\operatorname{dim} A-k$ at all of its points. We denote the latter set by $\Sigma(M, F)$.

For every face $\theta \subset \tau^{\vee}$, we can choose a closed regular subvariety $M_{\theta} \subset(\mathbb{C} \backslash 0)^{k}$ such that $\Sigma\left(M_{\theta}\right) \cap \mathbb{C}[A(\theta)]$ is dense in $\Sigma \cap \mathbb{C}[A(\theta)]$. Let $T_{\theta}$ be the orbit of the variety $\mathbb{T}^{\tau}$, corresponding to the face $\theta$, then the restriction of the map $\mathcal{F}$ to this orbit is a map $\mathcal{F}_{\theta}: T_{\theta} \rightarrow \mathbb{C}[A(\theta)]$. Proposition 5.4 for $\left(h_{1}, \ldots, h_{p}\right)=\mathcal{F}_{\theta}, S=\overline{\Sigma(M)_{\theta}} \cap$ $\mathbb{C}[A(\theta)]$ and Corollary 5.5 for $\left(h_{1}, \ldots, h_{p}\right)=\mathcal{F}_{\theta}, S=\Sigma(M)_{\theta} \cap \mathbb{C}[A(\theta)]$ imply the following:

(4) If the leading coefficients of the functions $f_{a}, a \in A$, are in general position, then the set $\Sigma(M, F) \cap T_{\theta}$ is dense in $\Sigma_{0}(F) \cap T_{\theta}$, and its codimension in $T_{\theta}$ is equal to $1+\operatorname{codim} M+\operatorname{dim} A(\theta)-k$, which is greater than $1+\operatorname{codim} M+\operatorname{dim} A-$ $k+\operatorname{dim} \theta-n$ under the assumption of Proposition 5.3. 
Since, by (3) and (4), the codimension of $\Sigma(M, F) \cap T_{\theta}$ in the toric variety $\mathbb{T}^{\tau}$ is greater than the codimension $\Sigma(M, F)$ in $\mathbb{T}^{\tau}$ at every point of $\Sigma(M, F) \cap T_{\theta}$, then $\Sigma(M, F) \cap T_{\theta}$ is contained in the closure of $\Sigma(M, F) \backslash T_{\theta}$. The inclusions

$$
\overline{\Sigma_{0}(F) \cap T_{\theta}}=\overline{\Sigma(M, F) \cap T_{\theta}} \subset \overline{\Sigma(M, F) \backslash T_{\theta}} \subset \overline{\Sigma(F)} \subset \overline{Z(F)}
$$

prove Proposition 5.3.

Example 5.9 Note that the inclusion $\overline{\Sigma_{0}(F) \cap T_{\theta}} \subset \overline{\Sigma(F)}$ and the statement of Proposition 5.3 may fail if $\operatorname{dim} A(\theta)=\operatorname{dim} A+\operatorname{dim} \theta-n$. For example, let $\mathbb{T}^{\tau}$ be the space $\mathbb{C}^{3}$ with coordinates $x, y, z$, and let $T_{\theta}$ be the torus $\{x \neq 0, y \neq 0, z=0\}$. Pick generic linear functions $l_{1}, l_{2}, l_{3}, m_{1}, m_{2}$ of the variables $x$ and $y$, and choose the face $A$ and the functions $f_{a}, a \in A$ as follows (each function $f_{a}(x, y, z)$ is written near the corresponding point $a \in A$ ):

$$
A\left\{\begin{array}{ccc}
\bullet m_{1}(x, y) & z m_{2}(x, y) \\
l_{1}(x, y) & l_{2}(x, y) & l_{3}(x, y) \\
\bullet & \bullet & \bullet
\end{array}\right\} A(\theta)
$$

Then the sets $\overline{\Sigma_{0}(F) \cap T_{\theta}}$ and $\overline{\Sigma(F)} \cap T_{\theta}$ are given by the equations

$$
l_{1}^{2}-l_{1} l_{3}=0 \quad \text { and } \quad l_{1} m_{1}^{2}-l_{2} m_{1} m_{2}+l_{3} m_{2}^{2}=0
$$

in $x$ and $y$, and hence do not intersect.

\subsection{Newton Polyhedra of Discriminants of Hypersurfaces}

If the Newton polyhedra $\Delta_{a}$ of functions $f_{a}, a \in A \subset \mathbb{Z}^{k}$, on the affine toric variety $\mathbb{T}^{\tau}$ are given, and the leading coefficients of these functions satisfy a certain condition of general position, then the Newton polyhedron of the discriminant $D_{F}$, where $F(x, y)=\sum_{a \in A} f_{a}(x) y^{a}$, depends only on the Newton polyhedra of these functions, not on the coefficients. Theorem 5.10 and Proposition 5.11 below solve the following problem:

Express the Newton polyhedron and leading coefficients of the discriminant $D_{F}$ in terms of the Newton polyhedra and leading coefficients of the functions $f_{a}$, provided that the leading coefficients are in general position.

Newton Polyhedron of the Discriminant The Minkowski integral $\int \Delta \subset \mathbb{R}^{n}$ of a polyhedron $\Delta \subset \mathbb{R}^{n} \oplus \mathbb{R}^{k}$ and combinatorial Euler obstructions $e^{A^{\prime}, A}$ and Milnor numbers $c^{A^{\prime}, A}$ are introduced in Sect. 2. Recall that a face of a set $A \subset \mathbb{Z}^{k}$ is the intersection of $A$ with a face of its convex hull, and $\operatorname{dim} A$ is the dimension of its convex hull. For a face $A^{\prime}$ of the set $A \subset \mathbb{Z}^{k}$, we denote the convex hull of the union $\bigcup_{a \in A^{\prime}} \Delta_{a} \times\{a\}$ by $\Delta\left(A^{\prime}\right)$; this is an unbounded face of $\Delta=\Delta(A)$. 


\section{Theorem 5.10}

(1) If the Newton polyhedra of the functions $f_{a}$ are given, and the leading coefficients of these functions are in general position in the sense of Definition 4.6, then the Newton polyhedron of the discriminant $D_{F}$ equals

$$
\mathcal{N}_{\Delta}^{A}=\sum_{A^{\prime} \subset A} e^{A^{\prime}, A} \cdot \int \Delta\left(A^{\prime}\right),
$$

where $A^{\prime}$ runs over all faces of $A$, including $A^{\prime}=A$.

(2) If the leading coefficients of the functions $f_{a}$ are arbitrary, then the Newton polyhedron of the discriminant $D_{F}$ is contained in $\mathcal{N}_{\Delta}^{A}$.

The proof is given in Sect. 5.5, and an example of application is given in Sect. 6.6. Note that Definition 4.6 is not the weakest possible condition of general position for this theorem. The weakest one (very complicated to verify though) can be extracted from Proposition 5.11 below; see the subsequent discussion.

Leading Coefficients of the Discriminant Let $\Gamma$ be a face of the polyhedron $\Delta$, denote the set of all $a \in A$, such that $\Gamma$ intersects $\Delta_{a} \times a$, by $A_{\Gamma}$, and denote the minimal face of $A$, containing $A_{\Gamma}$, by $\bar{A}_{\Gamma}$. For a bounded face $\widetilde{\Gamma}$ of another polyhedron, define the number $d^{\widetilde{\Gamma}, \Gamma}$ as $\sum_{\Gamma^{\prime}} c^{A_{\Gamma}, A_{\Gamma^{\prime}}} \cdot e^{\bar{A}_{\Gamma^{\prime}}, A}$, where $\Gamma^{\prime}$ runs over all bounded compatible with $\widetilde{\Gamma}$ faces of $\Delta$ such that $\Gamma^{\prime} \supset \Gamma$ and $\operatorname{dim} A_{\Gamma^{\prime}}=\operatorname{dim} \bar{A}_{\Gamma^{\prime}}$. Defining the value $D_{F^{\Gamma}}(x)$ as $R_{A_{\Gamma}}\left(F^{\Gamma}(x, \cdot)\right)$ for $x \in(\mathbb{C} \backslash 0)^{n}$, the Laurent polynomial $D_{F^{\Gamma}}$ on $(\mathbb{C} \backslash 0)^{n}$ depends only on leading coefficients of the functions $f_{a}, a \in A_{\Gamma}$.

\section{Proposition 5.11}

(1) For every bounded face $\widetilde{\Gamma}$ of the expected Newton polyhedron $\mathcal{N}_{\Delta}^{A}$ of the discriminant $D_{F}$,

$$
D_{F}^{\widetilde{\Gamma}}=\prod_{\Gamma}\left(D_{F \Gamma}\right)^{d^{\widetilde{\Gamma}, \Gamma}},
$$

where $\Gamma$ runs over all bounded faces of the polyhedron $\Delta$.

(2) Every multiplier $\left(D_{F^{\Gamma}}\right)^{d^{\widetilde{\Gamma}}, \Gamma}$ on the right-hand side of this equality is a polynomial (i.e., $d^{\widetilde{\Gamma}}, \Gamma \geqslant 0$ whenever the polynomial $D_{F} \Gamma$ is of positive degree).

In particular, the leading coefficients of the discriminant $D_{F}$ only depend on those of the functions $f_{a}, a \in A$. The proof is given in Sect. 5.5.

An assumption of general position for leading coefficients would be redundant in this statement: if the leading coefficients of the functions $f_{a}, a \in A$, are degenerate enough, then both parts of the equality become identically zero simultaneously. In particular, the Newton polyhedron of $D_{F}$ equals $\mathcal{N}_{\Delta}^{A}$ (i.e., $D_{F}$ has nonzero coefficients of the monomials, corresponding to the vertices of $\mathcal{N}_{\Delta}^{A}$ ) if and only if the following condition is satisfied for all $\widetilde{\Gamma}$ and $\Gamma$ : if $\widetilde{\Gamma}$ is a vertex, and $d^{\widetilde{\Gamma}}, \Gamma>0$, then $D_{F^{\Gamma}}$ is not identically zero. Note that the coefficient $d^{\widetilde{\Gamma}}, \Gamma$ is complicated to compute, and no simple combinatorial criterion for its positivity (i.e., for divisibility $D_{F}^{\widetilde{\Gamma}}$ 
by $D_{F^{\Gamma}}$ ) is known. See, for example, Theorem 15 in [4] for one important special case.

Degree of A-discriminants The Gelfand-Kapranov-Zelevinsky discriminant $D_{A}$ is a special case of the discriminant $D_{F}$ with $\mathbb{T}^{\tau}=\mathbb{C}[A]$. In this case, the discriminant is homogeneous, and its Newton polyhedron $\mathcal{N}$ is contained in the space $\mathbb{R}^{A}$, whose integer lattice consists of monomials of the form $\prod_{a \in A} c_{a}^{\lambda_{a}}$ in coefficients of the indeterminate polynomial $\sum_{a \in A} c_{a} x^{a} \in \mathbb{C}[A]$. We consider $\lambda_{a}, a \in A$, as a system of coordinates on $\mathbb{R}^{A}$, denote $\sum_{a} \lambda_{a}$ by $l$, and note that the degree of the discriminant $D_{A}$ is the minimal value of $l$ on $\mathcal{N}$.

Computing $\mathcal{N}$ by Theorem 5.10 and then $\left.\min l\right|_{\mathcal{N}}$ by Proposition 2.10 in this case, we get the following formula for the degree of $D_{A}$. Let $A^{\prime}$ be a face of $A$, and let $M \subset \mathbb{R}^{k}$ be the vector space, parallel to the affine span of $A^{\prime}$. We choose the volume form $\mu$ on $M$ such that the volume of $M /\left(M \cap \mathbb{Z}^{k}\right)$ equals $(\operatorname{dim} M)$ !, and denote the $\mu$-volume of the convex hull of $A^{\prime}$ by $\operatorname{Vol} A^{\prime}$.

Definition 5.12 Define the number $\operatorname{deg} A$ as the sum

$$
\sum_{A^{\prime} \subset A}^{\text {red }} e^{A^{\prime}, A} \cdot\left(\operatorname{dim} A^{\prime}+1\right) \operatorname{Vol}\left(A^{\prime}\right)
$$

over all faces $A^{\prime}$ of the set $A$, including $A^{\prime}=A$.

\section{Corollary 5.13 [20]}

(1) $\operatorname{deg} D_{A}=\operatorname{deg} A$.

(2) $D_{A}$ is a constant if and only if $\operatorname{deg} A=0$.

Remark A useful generalization of this fact for discriminants of higher codimension is proved in [20], based on a totally different technique (the Ernström formula [7]). Amazingly, that technique also ends up with Euler obstructions of toric varieties, which suggests that the two techniques could be unified. In particular, it would be interesting to find a common generalization of Theorem 5.10 and the Ernström formula, which would, for instance, compute the tropicalization of the dual of an arbitrary projective variety $V$ in terms of Euler obstructions of truncations of $V$.

\subsection{Proofs of Theorem 5.10 and Proposition 5.11}

Applying the Gelfand-Kapranov-Zelevinsky decomposition (Proposition 3.10) to the definition of reach discriminant (Definition 4.5), we have the following relation between discriminants and reach discriminants (see Definition 4.5) for every face $A^{\prime} \subset A$ :

$$
E_{F^{\Delta\left(A^{\prime}\right)}}=\prod_{A^{\prime \prime}}\left(D_{F^{\Delta\left(A^{\prime \prime}\right)}}\right)^{c^{A^{\prime \prime}, A^{\prime}},}
$$


where $A^{\prime \prime}$ runs over all faces of $A^{\prime}$, including $A^{\prime \prime}=A^{\prime}$. Inverting the formulas $\left(* A_{A^{\prime}}\right)$ by induction on the dimension of $A^{\prime}$, we have the following relations:

$$
D_{F \Delta\left(A^{\prime}\right)}=\prod_{A^{\prime \prime}}\left(E_{F \Delta\left(A^{\prime \prime}\right)}\right)^{e^{A^{\prime \prime}, A^{\prime}}},
$$

where $A^{\prime \prime}$ runs over all faces of $A^{\prime}$, including $A^{\prime \prime}=A^{\prime}$.

In more detail, assume that we have already obtained the formulas $\left(* *_{B}\right)$ for all faces $B$ of dimension less than $p$. Then, for every $A^{\prime}$ of dimension $p$, rewriting the formula $\left(* A^{\prime}\right)$ as $D_{F^{\Delta\left(A^{\prime}\right)}}=E_{F^{\Delta\left(A^{\prime}\right)}} \cdot \prod_{A^{\prime \prime} \neq A^{\prime}}\left(D_{F^{\Delta\left(A^{\prime \prime}\right)}}\right)^{-c^{A^{\prime \prime}, A^{\prime}}}$, expressing $D_{F^{\Delta\left(A^{\prime \prime}\right)}}$ in terms of $E_{F \Delta\left(A^{\prime \prime}\right)}$ by the formulas $\left(* *_{B}\right)$ on the right-hand side, and collecting similar multipliers, we obtain the formula $\left(* * A^{\prime}\right)$.

Informally speaking, if we consider the formal logarithm of the formulas $\left(*_{A^{\prime}}\right)$ to pass to the additive notation instead of the multiplicative one, then the vector of logarithms $\ln E_{F^{\Delta\left(A^{\prime}\right)}}, A^{\prime} \subset A$, equals the matrix with entries $c^{A^{\prime \prime}, A^{\prime}}$ times the vector of logarithms $\ln D_{F^{\Delta\left(A^{\prime}\right)}}, A^{\prime} \subset A$. Inverting the matrix, we obtain the logarithm of the formulas $\left(* * A_{A^{\prime}}\right)$.

In particular, $D_{A}(\varphi)=\prod_{A^{\prime}}\left(E_{A^{\prime}}\left(\varphi^{A^{\prime}}\right)\right)^{e^{A^{\prime}, A}}$.

Proof of Theorem 5.10 To prove Part 1, apply Proposition 4.7(1) and the identity $\left(* *_{A}\right)$ above.

To prove Part 2, consider a monomial curve $C: \mathbb{C} \rightarrow \mathbb{T}^{\tau}$, corresponding to an arbitrary positive integer linear function $\gamma: \tau^{\vee} \rightarrow \mathbb{R}_{>0}$; by definition, $C(t)=$ $\left(h_{1} t^{\gamma_{1}}, \ldots, h_{n} t^{\gamma_{n}}\right) \in(\mathbb{C} \backslash 0)^{n}$, where $\gamma_{1}, \ldots, \gamma_{n}$ are the coefficients of the linear function $\gamma$, and the coefficients $h_{1}, \ldots, h_{n}$ are generic. Then $D_{F}(C(t))$ is a germ of a meromorphic function of one variable $t$, and the order of its zero or pole is equal to the minimal value of the function $\gamma$ on the Newton polyhedron of $D_{F}$. Since this order of zero depends upper-semicontinuously on $F$, so does the Newton polyhedron of $D_{F}$.

\section{Proof of Proposition 5.11}

Part 1 For every face $A^{\prime}$ of the set $A$, choose a face $\widetilde{\Gamma}\left(A^{\prime}\right)$ of the polyhedron $\int \Delta\left(A^{\prime}\right)$, such that these faces $\widetilde{\Gamma}\left(A^{\prime}\right)$ together with $\widetilde{\Gamma}$ form a compatible collection. Represent the discriminant $D_{F}$ as a product of reach discriminants by formula $\left(*_{A}\right)$ above, then

$$
D_{F}^{\widetilde{\Gamma}}=\prod_{A^{\prime}}\left(E_{F^{\Delta\left(A^{\prime}\right)}}^{\widetilde{\Gamma}\left(A^{\prime}\right)}\right)^{e^{A^{\prime}, A}} .
$$

By Proposition 4.7(2), represent every truncated reach discriminant $E_{F^{\Delta\left(A^{\prime}\right)}}^{\widetilde{\Gamma}\left(A^{\prime}\right)}$ on the right-hand side as a product of reach discriminants of the form $E_{F^{\Gamma^{\prime}}}, \Gamma^{\prime} \subset \Delta\left(A^{\prime}\right)$. Finally, represent each of these reach discriminants as a product of discriminants by formula $\left(* A_{\Gamma^{\prime}}\right)$ above. 
Part 2 If the set $A_{\Gamma}$ is dual defect, then identically $D_{F^{\Gamma}}=1$, and the sign of the exponent $d^{\widetilde{\Gamma}}, \Gamma$ on the right-hand side of the identity in the statement of Proposition 5.11(1) is not important. Otherwise, we have the following.

Lemma 5.14 If the set $A_{\Gamma}$ is not dual defect, then $d^{\widetilde{\Gamma}}, \Gamma \geqslant 0$ for every bounded face $\widetilde{\Gamma} \subset \mathcal{N}_{\Delta}^{A}$.

Proof Let $A_{\Gamma}$ consist of points $a_{1}, \ldots, a_{N}$, and let $D_{A_{\Gamma}}\left(u_{1}, \ldots, u_{N}\right)$ be the value of the discriminant $D_{A_{\Gamma}}$ at the polynomial $\sum_{i} u_{i} t^{a_{i}} \in \mathbb{C}\left[A_{\Gamma}\right]$. Consider both sides of the identity in the statement of Proposition 5.11(1) as polynomials of the leading coefficients of the functions $f_{a}, a \in A$, with a fixed value of the variable $x \in(\mathbb{C} \backslash 0)^{n}$. Then

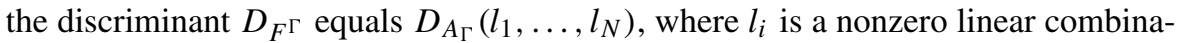
tion of leading coefficients of the function $f_{a_{i}}$. Since $D_{A_{\Gamma}}$ is a power of a homogeneous irreducible polynomial that nontrivially depends on all the variables $u_{1}, \ldots, u_{N}$ (by Lemmas 3.8 and 3.21), so does $D_{F^{\Gamma}}=D_{A_{\Gamma}}\left(l_{1}, \ldots, l_{N}\right)$ : it is a power of a homogeneous irreducible polynomial that nontrivially depends on all the coefficients of $F^{\Gamma}$ and does not depend on other leading coefficients of the functions $f_{a}, a \in A$.

Thus, if $d^{\widetilde{\Gamma}, \Gamma}$ were negative for some $\Gamma$, then the multiplier $\left(D_{F^{\Gamma}}\right)^{d^{\widetilde{\Gamma}}, \Gamma}$ could not be canceled by other multipliers on the right-hand side of the equality in the statement of Proposition 5.11(1), and the right-hand side would be a rational function with a nontrivial denominator. But this is impossible because the left-hand side is a polynomial.

\section{Discriminants of Complete Intersections}

In this section, we generalize the results of the previous two sections to discriminants of projections of analytic complete intersections.

The discriminant is defined in the first subsection. In Sect. 6.2, we reduce the study of its Newton polyhedron and leading coefficients to the case of projections of hypersurfaces, which is studied in the previous section. In some important special cases (Sects. 6.3 and 6.4), this leads to an explicit answer. An example of a computation of such an answer is given in the last subsection. We also consider an alternative definition of the discriminant in Sect. 6.5.

\subsection{Discriminants of Complete Intersections}

Let $\tau \subset\left(\mathbb{R}^{n}\right)^{*}$ be a convex $n$-dimensional rational polyhedral cone that does not contain a line, and let $A_{0}, \ldots, A_{l}, l \leqslant k$, be finite sets in $\mathbb{Z}^{k}$. For every $i=0, \ldots, l$, $a \in A_{i}$, let $f_{a, i}$ be a germ of a meromorphic function on the toric variety $\mathbb{T}^{\tau}$ with no poles in the maximal torus. Define the germ of a function $F_{i}$ on $\mathbb{T}^{\tau} \times(\mathbb{C} \backslash 0)^{k}$ by the formula

$$
F_{i}(x, y)=\sum_{a \in A_{i}} f_{a, i}(x) y^{a} \quad \text { for } x \in \mathbb{T}^{\tau}, y \in(\mathbb{C} \backslash 0)^{k},
$$

and denote the number $D_{A_{0}, \ldots, A_{l}}^{\mathrm{red}}\left(F_{0}(x, \cdot), \ldots, F_{l}(x, \cdot)\right)$ by $D_{F_{0}, \ldots, F_{l}}^{\text {red }}(x)$ (see Sect. 3.4 for the definition of the discriminant $\left.D_{A_{0}, \ldots, A_{l}}^{\text {red }}\right)$. 
Definition 6.1 The germ of the function $D_{F_{0}, \ldots, F_{l}}^{\text {red }}$ on the affine toric variety $\left(\mathbb{T}^{\tau}, O\right)$ is called the discriminant of the projection of the complete intersection $F_{0}=\cdots=$ $F_{l}=0$ to $\mathbb{T}^{\tau}$.

The discriminant has the expected geometric meaning if the leading coefficients are in general position. Namely, denote the set $\left\{x \in(\mathbb{C} \backslash 0)^{n} \mid D_{F_{0}, \ldots, F_{l}}^{\text {red }}(x)=0\right\}$ by $Z\left(F_{0}, \ldots, F_{l}\right)$, and consider the set $\Sigma\left(F_{0}, \ldots, F_{l}\right)$ of all $x \in(\mathbb{C} \backslash 0)^{n}$, such that $(0, \ldots, 0)$ is a singular value of the map $\left(F_{0}(x, \cdot), \ldots, F_{l}(x, \cdot)\right):(\mathbb{C} \backslash 0)^{k} \rightarrow \mathbb{C}^{l+1}$.

Proposition 6.2 Suppose that the Newton polyhedra of the functions $f_{a, i}, a \in A_{i}$, are given, and the leading coefficients of these functions are in general position. Then

(1) the union of codimension 1 components of the closure $\overline{\Sigma\left(F_{0}, \ldots, F_{l}\right)}$ equals $\overline{Z\left(F_{0}, \ldots, F_{l}\right)}$.

(2) If, in addition, the collection $A_{0}, \ldots, A_{k}$ is not dual defect (for example, if it satisfies assumptions of Proposition 3.24), then

$$
\overline{\Sigma\left(F_{0}, \ldots, F_{l}\right)}=\overline{Z\left(F_{0}, \ldots, F_{l}\right)},
$$

and, in particular, $\Sigma\left(F_{0}, \ldots, F_{l}\right)$ is a hypersurface.

This can be extended from the maximal torus $(\mathbb{C} \backslash 0)^{n}$ to the toric variety $\mathbb{T}^{\tau}$ in the same way as in Proposition 5.2 (see Proposition 5.3 for the notation). Consider the set $\Sigma_{0}\left(F_{0}, \ldots, F_{l}\right)$ of all $x \in \mathbb{T}^{\tau}$, such that $(0, \ldots, 0)$ is a singular value of the map $\left(F_{0}(x, \cdot), \ldots, F_{l}(x, \cdot)\right):(\mathbb{C} \backslash 0)^{k} \rightarrow \mathbb{C}^{l+1}$.

Proposition 6.3 Suppose that the functions $f_{a, i}, a \in A_{i}$, are holomorphic, their Newton polyhedra are such that $A_{j}(\theta) \neq \varnothing$ for every codimension 1 face $\theta \subset \tau^{\vee}$ and $j=0, \ldots, l$, and their leading coefficients are in general position. Then

(1) the union of all codimension 1 components of $\overline{\Sigma_{0}\left(F_{0}, \ldots, F_{l}\right)}$ equals $\overline{Z\left(F_{0}, \ldots, F_{l}\right)}$.

(2) If, in addition, the collection $A_{0}, \ldots, A_{k}$ is not dual defect, and $\operatorname{dim} \sum_{j} A_{j}(\theta)>$ $\operatorname{dim} L+\operatorname{dim} \theta-n$ for every $\theta \neq \tau^{\vee}$, then

$$
\overline{\Sigma_{0}\left(F_{0}, \ldots, F_{l}\right)}=\overline{Z\left(F_{0}, \ldots, F_{l}\right)},
$$

and, in particular, $\Sigma_{0}\left(F_{0}, \ldots, F_{l}\right)$ is a hypersurface.

Since the proof of these facts is the same as for Propositions 5.2 and 5.3, with the exception of more complicated notation coming from $l>0$, we omit it.

\subsection{Newton Polyhedra of Discriminants of Complete Intersections}

The study of the Newton polyhedron and leading coefficients of the discriminant $D_{F_{0}, \ldots, F_{l}}^{\text {red }}$ can be reduced to the case $l=0$ (which is studied in the previous section) by the Cayley trick, which represents $D_{F_{0}, \ldots, F_{l}}^{\text {red }}$ as a product of discriminants of the 
form $D_{G_{J}}^{\text {red }}$ for linear combinations $G_{J}=\sum_{j \in J} \lambda_{j} F_{j}$ with indeterminate coefficients $\lambda_{j}$, where $J$ runs over certain subsets of $\{0, \ldots, l\}$.

More precisely, define the function $G_{J}$ on $\mathbb{T}^{\tau} \times(\mathbb{C} \backslash 0)^{k} \times(\mathbb{C} \backslash 0)^{l+1}$ as $\sum_{j \in J} \lambda_{j} F_{j}$, where $\lambda_{0}, \ldots, \lambda_{l}$ are coordinates on $(\mathbb{C} \backslash 0)^{l+1}$. Let $e_{0}, \ldots, e_{l}$ be the standard basis in $\mathbb{Z}^{l+1}$, and denote the set $\bigcup_{j \in J}^{\text {red }} A_{j} \times\left\{e_{j}\right\}$ by $A_{J} \subset \mathbb{Z}^{k} \oplus \mathbb{Z}^{l+1}$. Then, for every $x \in \mathbb{T}^{\tau}$, the polynomial $G_{J}(x, \cdot)$ on $(\mathbb{C} \backslash 0)^{k} \times(\mathbb{C} \backslash 0)^{l+1}$ is contained in $\mathbb{C}\left[A_{J}\right]$, and the discriminant $D_{G_{J}}^{\mathrm{red}}$ is defined by the formula $D_{G_{J}}^{\mathrm{red}}(x)=D_{A_{J}}^{\mathrm{red}}\left(G_{J}(x, \cdot)\right)$.

These discriminants are related to the desired one as follows. For every $J \subset$ $\{0, \ldots, l\}$, denote the difference $\operatorname{dim} \sum_{j \in J} A_{j}-|J|$ by codim $J$.

Theorem 6.4 (Cayley trick) The discriminant $D_{F_{0}, \ldots, F_{l}}^{\mathrm{red}}, 0<l<k$, equals the product of the discriminants $D_{G_{J}}^{\text {red }}$ over all subsets $J \subset\{0, \ldots, l\}$, such that $\operatorname{codim} J \leqslant$ codim $J^{\prime}$ for every $J^{\prime} \supset J$.

This is Theorem 3.31 in the new notation. To describe the Newton polyhedra and leading coefficients of the discriminants $D_{G_{J}}^{\text {red }}$, and therefore those of $D_{F_{0}, \ldots, F_{l}}^{\text {red }}$, we can apply Theorem 5.10 and Proposition 5.11 to the functions $G_{J}$ under an appropriate condition of general position for their leading coefficients (see Definition 4.6). In many cases, the result of this computation can be written as an explicit formula for the Newton polyhedron of $D_{F_{0}, \ldots, F_{l}}^{\text {red }}$; see, for example, Theorem 6.10 below. The aforementioned condition of general position can be formulated in terms of leading coefficients of the functions $F_{0}, \ldots, F_{l}$ as follows.

We denote the Newton polyhedron of $f_{a, i}$ by $\Delta_{a, i}$, and define the Newton polyhedron $\Delta_{i}$ of the function $F_{i}$ as the convex hull of the set $\bigcup_{a \in A_{i}} \Delta_{a, i} \times\{a\} \subset \mathbb{R}^{n} \oplus \mathbb{R}^{k}$; then $F_{i}(z)$ can be represented as a power series $\sum_{b \in \Delta_{i}} c_{b, i} z^{b}$ for $z \in(\mathbb{C} \backslash 0)^{n} \times$ $(\mathbb{C} \backslash 0)^{k}$. If $\Gamma$ is a face of $\Delta_{i}$, then we denote the function $\sum_{b \in \Gamma} c_{b, i} z^{b}$ by $F_{i}^{\Gamma}$.

Definition 6.5 The leading coefficients of the functions $f_{a, i}, a \in A_{i}$, are said to be in general position if, for every sequence $0 \leqslant i_{1}<\cdots<i_{q} \leqslant l$ and every collection of compatible bounded faces $\Gamma_{i_{j}} \subset \Delta_{i_{j}}$ (see Definition 2.13), such that the restriction of the projection $\mathbb{R}^{n} \oplus \mathbb{R}^{k} \rightarrow \mathbb{R}^{k}$ to $\Gamma_{i_{1}}+\cdots+\Gamma_{i_{q}}$ is injective, $(0, \ldots, 0)$ is a regular value of the polynomial map $\left(F_{i_{1}}^{\Gamma_{i_{1}}}, \ldots, F_{i_{q}}^{\Gamma_{i_{q}}}\right):(\mathbb{C} \backslash 0)^{n+k} \rightarrow \mathbb{C}^{q}$.

\subsection{The Case of Analogous Newton Polyhedra}

Under some additional assumptions on the sets $A_{0}, \ldots, A_{l}$, the Cayley trick allows us to explicitly compute the Newton polyhedron of the discriminant of a complete intersection as follows.

Definition 6.6 Let $A^{\prime}$ be a face of a finite set $A \subset \mathbb{R}^{k}$, and let $L$ be the vector subspace in $\mathbb{R}^{k}$, parallel to the affine span of $A^{\prime}$. The $A^{\prime}$-link of $A$ is the (nonconvex and nonclosed) polyhedron $\widetilde{A} \backslash \widetilde{A}^{\prime} \subset \mathbb{R}^{k} / L$, where $\widetilde{A}$ and $\widetilde{A}^{\prime}$ are the convex hulls of the images of the sets $A$ and $A \backslash A^{\prime}$ under the projection $\mathbb{R}^{k} \rightarrow \mathbb{R}^{k} / L$. 
Definition 6.7 Finite sets $A$ and $B$ in $\mathbb{R}^{k}$ are said to be analogous if there is a oneto-one correspondence between the posets of their faces, such that, for every pair of corresponding faces $A^{\prime} \subset A$ and $B^{\prime} \subset B$, the $A^{\prime}$-link of $A$ equals the $B^{\prime}$-link of $B$ up to a parallel translation (in particular, the affine spans of $A^{\prime}$ and $B^{\prime}$ are parallel to the same subspace $L \subset \mathbb{R}^{k}$ ).

\section{Example 6.8}

(1) If $A=B$, then $A$ and $B$ are analogous.

(2) If $P$ and $Q$ are analogous integer polyhedra (i.e., their dual fans coincide) and $k \in \mathbb{Z}$ is large enough, then the sets of integer points in $k P$ and $k Q$ are analogous. Note that those sets are not necessarily analogous for $k=1$. For example, the two sets on the picture in Sect. 2.5 have different links of their vertical faces.

Recall that the standard basis in $\mathbb{Z}^{l+1}$ is denoted by $e_{0}, \ldots, e_{l}$.

Lemma 6.9 If $A_{0}, \ldots, A_{l}$ in $\mathbb{Z}^{k}$ are analogous, then, for every collection of corresponding faces $A_{0}^{\prime} \subset A_{0}, \ldots, A_{l}^{\prime} \subset A_{l}$,

(1) $e^{A_{0}^{\prime}, A_{0}}=\cdots=e^{A_{l}^{\prime}, A_{l}}$,

(2) $A^{\prime}=\bigcup_{i=0}^{l^{\prime}} A_{i}^{\prime} \times\left\{e_{i}\right\}$ is a face of $A=\bigcup_{i=0}^{l} A_{i} \times\left\{e_{i}\right\}$, and $e^{A^{\prime}, A}=e^{A_{0}^{\prime}, A_{0}}$.

Proof Part 1 and Part 2 for $l^{\prime}=l$ follow by the fact that the Euler obstruction $e^{B^{\prime}, B}$ depends on the $B^{\prime}$-link of $B$ only (by definition, see Sect. 2.5). Since $e^{B^{\prime}, B}$ is the Euler obstruction of the $B$-toric variety at a point of its $B^{\prime}$-orbit (Proposition 2.27), and since Euler obstruction is a local topological invariant, then $e^{A^{\prime}, A}$ does not depend on $l^{\prime}$, and it is enough to prove Part 2 for $l^{\prime}=l$.

In the notation of Sect. 6.1, let $M$ be the lattice, generated by pairwise differences of points of the set $A_{0}+\cdots+A_{l}$, and let $\Delta_{0}, \ldots, \Delta_{l}$ be the Newton polyhedra of the functions $F_{0}, \ldots, F_{l}$. Recall that we denote the mixed fiber polyhedron of polyhedra $P_{1}, \ldots, P_{q}$ by the monomial $P_{1} \ldots P_{q}$.

\section{Theorem 6.10}

(1) If the leading coefficients of the functions $f_{a, i}, a \in A_{i}$, are in general position in the sense of Definition 6.5, and the sets $A_{0}, \ldots, A_{l}$ are analogous and not contained in an affine hyperplane, then the Newton polyhedron of the discriminant $D_{F_{0}, \ldots, F_{l}}^{\text {red }}$ equals

$$
\mathcal{N}=\frac{1}{\left|\mathbb{Z}^{k} / M\right|} \sum_{A_{0}^{\prime}, \ldots, A_{l}^{\prime}} e^{A_{0}^{\prime}, A_{0}} \sum_{\begin{array}{c}
a_{0}>0, \ldots, a_{l}>0 \\
a_{0}+\cdots+a_{l}=\operatorname{dim} A_{0}^{\prime}+1
\end{array}} \Delta_{0}\left(A_{0}^{\prime}\right)^{a_{0}} \cdots \Delta_{l}\left(A_{l}^{\prime}\right)^{a_{l}},
$$

where the collection $\left(A_{0}^{\prime}, \ldots, A_{l}^{\prime}\right)$ runs over all collections of corresponding faces $A_{0}^{\prime} \subset A_{0}, \ldots, A_{l}^{\prime} \subset A_{l}$, including $A_{0}^{\prime}=A_{0}, \ldots, A_{l}^{\prime}=A_{l}$.

(2) If the leading coefficients of the functions $f_{a, i}$ are arbitrary, then the Newton polyhedron of the discriminant $D_{F_{0}, \ldots, F_{l}}^{\mathrm{red}}$ is contained in $\mathcal{N}$. 
An example of application is given in Sect. 6.6.

Proof By Theorem 6.4, we have $D_{F_{0}, \ldots, F_{l}}^{\text {red }}=D_{F_{\{0, \ldots, l\}}}^{\text {red }}$. Thus, the Newton polyhedron of $D_{F_{0}, \ldots, F_{l}}^{\text {red }}$ is $\left|\mathbb{Z}^{k} / M\right|$ times smaller than the Newton polyhedron of $D_{F_{\{0, \ldots, l\}}}$. We compute the latter one by Theorem 5.10, and simplify the answer by Lemmas 6.9 and 2.12(2).

\subsection{The Case of Branched Coverings and Higher Additivity}

The assumptions of Theorem 6.10 can be significantly relaxed, especially for large $l$. We illustrate this for $l=k$ (elimination theory) and for $l=k-1$ (the projection of $F_{0}=\cdots=F_{l}=0$ onto $\mathbb{T}^{\tau}$ is typically a branched covering in this case). ables.

Let $\sigma_{m}\left(t_{1}, \ldots, t_{l}\right)$ be the symmetric function $\sum_{\substack{a_{1}>0, \ldots, a_{l}>0 \\ a_{1}+\cdots+a_{l}=m}} t_{1}^{a_{1}} \cdots t_{l}^{a_{l}}$ of formal vari-

Lemma 6.11 (Higher additivity)

$$
\begin{aligned}
\sigma_{m}\left(t_{0}+\tilde{t}_{0}, t_{1}, \ldots, t_{l}\right) \\
=\sum_{\mu=1}^{\infty} \sigma_{m}(\underbrace{t_{0}, \ldots, t_{0}}_{\mu}, \underbrace{\tilde{t}_{0}, \ldots, \tilde{t}_{0}}_{\mu-1}, t_{1}, \ldots, t_{l}) \\
\quad+\sigma_{m}(\underbrace{t_{0}, \ldots, t_{0}}_{\mu-1}, \underbrace{\tilde{t}_{0}, \ldots, \tilde{t}_{0}}_{\mu}, t_{1}, \ldots, t_{l}) \\
\quad+2 \sigma_{m}(\underbrace{t_{0}^{t_{0}, \ldots, t_{0}}}_{\mu}, \underbrace{\tilde{t}_{0}, \ldots, \tilde{t}_{0}}_{\mu}, t_{1}, \ldots, t_{l}) .
\end{aligned}
$$

Note that there are only finitely many nonzero terms (those for $2 \mu+k \leqslant m$ ) on the right-hand side. For $m=l+1$, the identity degenerates to $\left(t_{0}+\tilde{t}_{0}\right) t_{1} \cdots t_{l}=$ $t_{0} t_{1} \cdots t_{k}+\tilde{t}_{0} t_{1} \cdots t_{l}$. The proof is standard.

Let $\mathcal{M}_{\tau^{\vee}}\left(A_{0}\right)$ be the semigroup of all pairs of the form $(A, \Delta)$, such that the finite set $A \subset \mathbb{Z}^{k}$ is analogous to $A_{0} \subset \mathbb{Z}^{k}$, the polyhedron $\Delta$ is in $\mathcal{M}_{\tau^{\vee}}$, and its image under the projection $\mathbb{R}^{n} \oplus \mathbb{R}^{k} \rightarrow \mathbb{R}^{k}$ equals the convex hull of $A$ (this is a semigroup with respect to Minkowski addition of finite sets and polyhedra).

Definition 6.12 The higher mixed fiber polyhedron is the collection of symmetric functions

$$
\mathrm{HP}: \underbrace{\mathcal{M}_{\tau^{\vee}}\left(A_{0}\right) \times \cdots \times \mathcal{M}_{\tau}\left(A_{0}\right)}_{l+1} \rightarrow \mathcal{M}_{\tau \vee}(0)
$$

for $l \geqslant 0$, such that

$$
\text { (1) } \operatorname{HP}(\underbrace{(A, \Delta), \ldots,(A, \Delta)}_{l+1})=\sum_{A^{\prime} \subset A} e^{A^{\prime}, A}\left(\begin{array}{c}
\operatorname{dim} A^{\prime} \\
l
\end{array}\right) \int \Delta\left(A^{\prime}\right) \text {, }
$$


for every $(A, \Delta) \in \mathcal{M}_{\tau^{\vee}}\left(A_{0}\right)$ with $\operatorname{dim} A=k\left(A^{\prime}\right.$ runs over all faces of $A$ of dimension $l$ or greater $)$, and $\operatorname{HP}((A, \Delta), \ldots,(A, \Delta))=\tau^{\vee}$ for $\operatorname{dim} A<k$;

$$
\text { (2) } \begin{aligned}
& \operatorname{HP}\left(t_{0}+\tilde{t}_{0}, t_{1}, \ldots, t_{l}\right) \\
&=\sum_{\mu=1}^{\infty} \operatorname{HP}(\underbrace{t_{0}, \ldots, t_{0}}_{\mu}, \underbrace{\tilde{t}_{0}, \ldots, \tilde{t}_{0}}_{\mu-1}, t_{1}, \ldots, t_{l}) \\
&+\operatorname{HP}(\underbrace{t_{0}, \ldots, t_{0}}_{\mu-1}, \underbrace{\tilde{t}_{0}, \ldots, \tilde{t}_{0}}_{\mu}, t_{1}, \ldots, t_{l}) \\
&+2 \operatorname{HP}(\underbrace{t_{0}, \ldots, t_{0}}_{\mu}, \underbrace{\tilde{t}_{0}, \ldots, \tilde{t}_{0}}_{\mu}, t_{1}, \ldots, t_{l})
\end{aligned}
$$

for all pairs $t_{0}, \tilde{t}_{0}, t_{1}, \ldots, t_{l}$ in $\mathcal{M}_{\tau^{\vee}}\left(A_{0}\right)$.

By induction on $k-l$, these conditions uniquely define the function HP (at the base of the induction, for $l=k$, we have the definition of the mixed fiber polyhedron). On the other hand, by the lemma above, the polyhedron

$$
\begin{aligned}
& \operatorname{HP}\left(\left(A_{0}, \Delta_{0}\right), \ldots,\left(A_{l}, \Delta_{l}\right)\right) \\
& \quad=\sum_{A_{0}^{\prime}, \ldots, A_{l}^{\prime}} e^{A_{0}^{\prime}, A_{0}} \sum_{\substack{a_{0}>0, \ldots, a_{l}>0 \\
a_{0}+\cdots+a_{l}=\operatorname{dim} A_{0}^{\prime}+1}} \Delta_{0}\left(A_{0}^{\prime}\right)^{a_{0}} \cdots \Delta_{l}\left(A_{l}^{\prime}\right)^{a_{l}},
\end{aligned}
$$

with $\left(A_{0}^{\prime}, \ldots, A_{l}^{\prime}\right)$ running over all collections of corresponding faces $A_{0}^{\prime} \subset A_{0}, \ldots$, $A_{l}^{\prime} \subset A_{l}$, satisfies Definition 6.12. In particular, $\mathrm{HP}=0$ for $l>k$. We can now formulate Theorem 6.10 as follows.

Theorem 6.13 If $A_{0}, \ldots, A_{l}$ are analogous finite sets in $\mathbb{R}^{k}$, and $\sum_{i} A_{i} \times\{1\}$ generates $\mathbb{Z}^{k} \oplus \mathbb{Z}^{1}$, then, in the notation of Sect. 6.1, the Newton polyhedron of the discriminant $D_{F_{0}, \ldots, F_{l}}^{\text {red }}$ equals $\operatorname{HP}\left(\left(A_{0}, \Delta_{0}\right), \ldots,\left(A_{l}, \Delta_{l}\right)\right)$.

Unexpectedly, as soon as we formulate Theorem 6.10 in this form, it can be generalized to nonanalogous collections $A_{0}, \ldots, A_{l}$ in some cases (examples are Propositions 6.14 and 6.16 below), which motivates the following open question:

To what extent can one relax the assumption that the arguments of HP are analogous in Definition 6.12, so that the higher mixed fiber polyhedron still exists and Theorem 6.13 remains valid?

In Sect. 6.2, we computed the Newton polyhedron of the discriminant $D_{F_{0}, \ldots, F_{l}}^{\text {red }}$ of functions $F_{0}, \ldots, F_{l}$, whose leading coefficients are in general position. We denote this Newton polyhedron by $\mathcal{N}_{\Delta_{0}, \ldots, \Delta_{l}}^{A_{0}, \ldots, A_{l}}$, where $\Delta_{0}, \ldots, \Delta_{l}$ are the Newton polyhedra of the functions $F_{0}, \ldots, F_{l}$. The following description of $\mathcal{N}_{\Delta_{0}, \ldots, \Delta_{k}}^{A_{0}, \ldots, A_{k}}$ is equivalent to Theorem 6.10 for $l=k$ and analogous sets $A_{0}, \ldots, A_{k}$, but is valid for arbitrary sets $A_{0}, \ldots, A_{k}$. 
Proposition 6.14 Suppose that $l=k$, and the lattice $\mathbb{Z}^{k}$ is generated by pairwise differences of elements of $A_{i}$ for every $i=0,0^{\prime}, 1, \ldots, k$. Then Additivity: $\mathcal{N}_{\Delta_{0}+\Delta_{0^{\prime}}, \Delta_{1}, \ldots, \Delta_{k}}^{A_{0}+A_{0^{\prime}}, A_{1}, \ldots, A_{k}}=\mathcal{N}_{\Delta_{0}, \ldots, \Delta_{k}}^{A_{0}, \ldots, A_{k}}+\mathcal{N}_{\Delta_{0^{\prime}}, \Delta_{1}, \ldots, \Delta_{k}}^{A_{0^{\prime}}, A_{1}, \ldots, A_{k}}$. Unmixed case: If $\Delta_{0}=\cdots=\Delta_{k}$, and $A_{0}$ is not contained in a hyperplane, then $\mathcal{N}_{\Delta_{0}, \ldots, \Delta_{k}}^{A_{0}, \ldots, A_{k}}=\int \Delta_{0}$.

This is just another formulation of Theorem 4.3(1). We generalize this proposition to the case $l=k-1$ as follows.

Definition 6.15 Finite sets $A \subset \mathbb{Z}^{k}$ and $V \subset\left(\mathbb{Z}^{k}\right)^{*}$ are said to be compatible if

(1) $V$ contains the primitive external normal covector to every codimension 1 face of the convex hull of $A$

(2) for every linear function $v \in V$, the maximal and the next-to-the-maximal values of $v$ on $A$ differ by 1

(3) pairwise differences of elements of $A$ generate $\mathbb{Z}^{k}$

Note that, if $A_{1}$ and $A_{2}$ are compatible with the same $V$, it does not imply that $A_{1}$ and $A_{2}$ are analogous: the simplest example is $A_{1}=\{(0,0),(0,1),(1,0)\}$ and $A_{2}=\{(0,0),(0,-1),(-1,0)\}$.

Proposition 6.16 Suppose that $l=k-1$, and all the sets $\sum_{i \in I} A_{i}, I \subset\left\{0,0^{\prime}, 1, \ldots\right.$, $k-1\}$, are compatible with the same set $V \in\left(\mathbb{Z}^{k}\right)^{*}$. Then Higher additivity:

$$
\mathcal{N}_{\Delta_{0}+\Delta_{0^{\prime}}, \Delta_{1}, \ldots, \Delta_{k-1}}^{A_{0}+A_{0^{\prime}}, A_{1}, \ldots, A_{k-1}}=\mathcal{N}_{\Delta_{0}, \ldots, \Delta_{k-1}}^{A_{0}, \ldots, A_{k-1}}+\mathcal{N}_{\Delta_{0^{\prime}}, \Delta_{1}, \ldots, \Delta_{k-1}}^{A_{0^{\prime}}, A_{1}, \ldots, A_{k-1}}+2 \mathcal{N}_{\Delta_{0}, \Delta_{0^{\prime}}, \Delta_{1}, \ldots, \Delta_{k-1}}^{A_{0}, A_{0^{\prime}}, A_{1}, \ldots, A_{k-1}} ;
$$

Unmixed case: If $\Delta_{0}=\cdots=\Delta_{k-1}$, and $A_{0}$ has codimension 0 , then

$$
\mathcal{N}_{\Delta_{0}, \ldots, \Delta_{k-1}}^{A_{0}, \ldots, A_{k-1}}=k \int \Delta_{0}-\sum_{A^{\prime}} \int \Delta_{0}\left(A^{\prime}\right),
$$

where $A^{\prime}$ runs over all codimension 1 faces of $A_{0}$.

In the same way as for $l=k$, these two identities are enough to compute $\mathcal{N}_{\Delta_{0}, \ldots, \Delta_{k-1}}^{A_{0}, \ldots, A_{k-1}}$. The proof of Proposition 6.16 follows the same lines as for Theorem 6.10; the computations are not affected by the fact that the sets $A_{0}, \ldots, A_{k-1}$ may not be analogous under the assumptions above.

Remark Another way to prove additivity in Proposition 6.16 is to consider functions $F_{i}$ with generic leading coefficients and Newton polyhedra $\Delta_{i}$ for $i=$ $0,0^{\prime}, 1, \ldots, k-1$, and a function $F$ with generic leading coefficients and the Newton polyhedron $\Delta_{0}+\Delta_{0^{\prime}}$. Then, as $F$ tends to the product $F_{0} F_{0^{\prime}}$, the discriminant $D_{F, F_{1}, \ldots, F_{k-1}}$ tends to the product $D_{F_{0}, F_{1}, \ldots, F_{k-1}} D_{F_{0^{\prime}}, F_{1}, \ldots, F_{k-1}}\left(D_{F_{0}, F_{0^{\prime}}, F_{1}, \ldots, F_{k-1}}\right)^{2}$, as the following picture illustrates for $l=k-1=0$. 


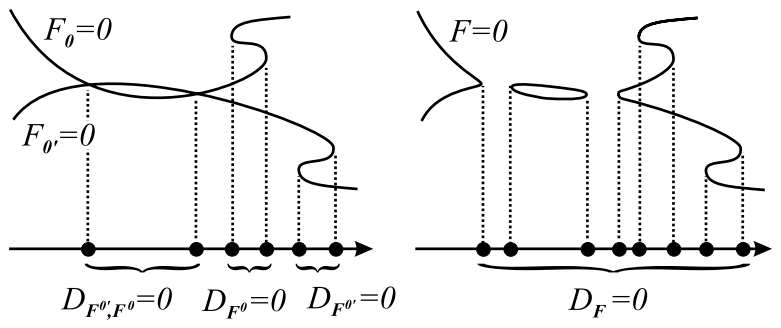

We omit a detailed proof of Proposition 6.16, because its only purpose is to provide a motivation for the question formulated after Theorem 6.13, but neither of the two mentioned ideas of the proof seem relevant to answer this question.

\subsection{Bifurcation Sets and Their Newton Polyhedra}

In this subsection, we study one more problem, similar to the one studied in the first part of this section. Namely, in the notation of Sect. 6.1, we study the minimal (closed) subset $S_{F_{0}, \ldots, F_{l}} \subset \mathbb{T}^{\tau}$, such that the restriction of the projection $\mathbb{T}^{\tau} \times(\mathbb{C} \backslash$ $0)^{k} \rightarrow \mathbb{T}^{\tau}$ to $\left\{F_{0}=\cdots=F_{l}=0\right\}$ is a fiber bundle outside of $S_{F_{0}, \ldots, F_{l}}$. It is called the bifurcation set of the projection. Note that, in contrast to $\left\{D_{F_{0}, \ldots, F_{l}}^{\text {red }}=0\right\}$, the set $S_{F_{0}, \ldots, F_{l}}$ takes into account "singularities at infinity" of fibers of the projection $\left\{F_{0}=\right.$ $\left.\cdots=F_{l}=0\right\} \rightarrow \mathbb{T}^{\tau}$. For details and examples, see Sect. 3.4 where the universal case of this problem is studied.

We are interested in the Newton polyhedron of the equation of $S_{F_{0}, \ldots, F_{l}}$, under the assumption that the Newton polyhedra of $F_{0}, \ldots, F_{l}$ are given, and their leading coefficients are in general position. If $\Delta_{0}, \ldots, \Delta_{l}$ are the Newton polyhedra of the functions $F_{0}, \ldots, F_{l}$, and the leading coefficients of these functions are in general position, then we denote the Newton polyhedron of the discriminant $D_{F_{0}, \ldots, F_{l}}^{\text {red }}$ by $\mathcal{N}_{\Delta_{0}, \ldots, \Delta_{l}}^{A_{0}, \ldots, A_{l}}$ (see Sect. 6.2 for its computation).

Theorem 6.17 If the collection $A_{0}, \ldots, A_{l}$ is $B$-nondegenerate (see Sect. 3.4), and the leading coefficients of the functions $f_{a, i}, a \in A_{i}$, are in general position in the sense of Definition 6.5, then

(1) the bifurcation set $S_{F_{0}, \ldots, F_{l}}$ is a hypersurface.

(2) Assigning appropriate positive multiplicities to the components of the hypersurface $S_{F_{0}, \ldots, F_{l}} \subset \mathbb{T}^{\tau}$ outside the maximal torus, it becomes a Cartier divisor, and the Newton polyhedron of its equation equals

$$
\sum_{A_{0}^{\prime}, \ldots, A_{l}^{\prime}} \mathcal{N}_{\Delta\left(A_{0}^{\prime}\right), \ldots, \Delta\left(A_{l}^{\prime}\right)}^{A_{0}^{\prime}, \ldots, A_{l}^{\prime}},
$$

where $A_{0}^{\prime}, \ldots, A_{l}^{\prime}$ runs over all collections of compatible faces of the sets $A_{0}, \ldots, A_{l}$, including $A_{0}^{\prime}=A_{0}, \ldots, A_{l}^{\prime}=A_{l}$.

Note that the first assumption in this statement can be omitted if Conjecture 3.28 is valid. 
The proof of Theorem 6.17 is based on the following idea. Define the function $H_{F_{0}, \ldots, F_{l}}$ on $\mathbb{T}^{\tau}$ as follows: $H_{F_{0}, \ldots, F_{l}}(x)=B_{A_{0}, \ldots, A_{l}}\left(F_{0}(x, \cdot), \ldots, F_{l}(x, \cdot)\right)$ (see Sect. 3.4 for the definition of the discriminant $\left.B_{A_{0}, \ldots, A_{l}}\right)$. Then $\left\{H_{F_{0}, \ldots, F_{l}}=0\right\}$ is the equation of $S_{F_{0}, \ldots, F_{l}}$ (the proof follows the same lines as the proof of Propositions 5.2 and 5.3), and equals the product $\prod_{A_{0}^{\prime}, \ldots, A_{l}^{\prime}} D_{F^{\Delta\left(A_{0}^{\prime}\right)}, \ldots, F^{\Delta\left(A_{l}^{\prime}\right)}}^{\text {red }}$, where $A_{0}^{\prime}, \ldots, A_{l}^{\prime}$ runs over all collections of compatible faces of the sets $A_{0}, \ldots, A_{l}$, including $A_{0}^{\prime}=$ $A_{0}, \ldots, A_{l}^{\prime}=A_{l}$, by Lemma 3.30 .

Corollary 6.18 If each of $A_{0}, \ldots, A_{l}$ is the set of integer lattice points in a Delzant polytope, these Delzant polytopes have the same dual fan, and the leading coefficients of the functions $f_{a, i}, a \in A_{i}$, are in general position in the sense of Definition 6.5, then $e^{A_{i}^{\prime}, A_{i}}=(-1)^{\operatorname{dim} A_{i}-\operatorname{dim} A_{i}^{\prime}}$ for every face $A_{i}^{\prime}$, and the Newton polyhedron of the equation of $S_{F_{0}, \ldots, F_{l}}$ equals

$$
\sum_{A_{0}^{\prime}, \ldots, A_{l}^{\prime}} \sum_{\substack{a_{0}>0, \ldots, a_{l}>0 \\ a_{0}+\cdots+a_{l}=k+1}} \Delta_{0}\left(A_{0}^{\prime}\right)^{a_{0}} \cdots \Delta_{l}\left(A_{l}^{\prime}\right)^{a_{l}},
$$

where $A_{0}^{\prime}, \ldots, A_{l}^{\prime}$ runs over all collections of compatible faces of the sets $A_{0}, \ldots, A_{l}$, including $A_{0}^{\prime}=A_{0}, \ldots, A_{l}^{\prime}=A_{l}$.

This is a corollary of Theorems 6.17, 6.10, and Proposition 3.29.

\subsection{Example and Computability Questions}

EXAMPLE. Consider the first coordinate in the torus $(\mathbb{C} \backslash 0)^{3}$ as the "height function" and the first coordinate line in $\mathbb{R}^{3}$ as the "vertical" line. We will compute the number of critical points of the restriction of the height function onto the curve $f=g=0$ and onto the surface $f=0$ for generic equations $f$ and $g$ with a given Newton polyhedron $\Delta \subset \mathbb{R}^{3}$. We can compute these numbers by the global version of Theorems 6.10 and 5.10 respectively with $k=2$ and $n=1$, because the number of critical points of the height function is the degree of the discriminant of the projection onto the vertical coordinate line. The answer is as follows.

Let $S_{1}, \ldots, S_{n}$ be the areas of vertical faces of $\Delta$, let $l_{1}, \ldots, l_{n}$ be the lengths of its vertical edges, and let $e_{1}, \ldots, e_{n}$ be the Euler obstructions of the projection of $\Delta$ to the horizontal plane at the corresponding vertices. Recall that the Euler obstruction of an integer polygon $P$ at its vertex $Q$ equals

$$
2-2 \cdot \text { area of } P \backslash P_{Q},
$$

where $P_{Q}$ is the convex hull of $\mathbb{Z}^{2} \cap P \backslash\{Q\}$ (see Example 2.26 for details), and note that the Euler obstruction of $P$ at all of its edges equals -1 . For instance, the Euler obstruction of the standard simplex at every vertex equals 1, while the Euler obstruction of the convex hull of the points $( \pm 1,0)$ and $(0,1)$ at every vertex equals 0 .

In this notation, the number of critical points of the restriction of the height function onto the curve $f=g=0$ equals

$$
12 \mathrm{Vol} \Delta-2 \sum_{i} S_{i} .
$$


The number of critical points of the restriction of the height function onto the surface $f=0$ equals

$$
6 \mathrm{Vol} \Delta-2 \sum_{i} S_{i}+\sum_{i} e_{i} l_{i} .
$$

To explain the first coefficient in the first of these answers informally, note that the desired critical points are solutions of the following system of equations:

$$
f=g=\operatorname{det}\left(\begin{array}{ll}
\frac{\partial f}{\partial y} & \frac{\partial f}{\partial z} \\
\frac{\partial g}{\partial y} & \frac{\partial g}{\partial z}
\end{array}\right)=0 .
$$

The Newton polyhedron of the first two equations is denoted by $\Delta$, thus the Newton polyhedron of the last equation "approximately" equals $2 \Delta$. Thus, if the Kouchnirenko-Bernstein formula were applicable to this system of the equations, then it would have approximately $6 \operatorname{MV}(\Delta, \Delta, 2 \Delta)=12 \operatorname{Vol}(\Delta)$ solutions. Although this illustrates why the coefficient in the first answer equals 12 , neither does the Newton polyhedron of the last solution equal $2 \Delta$ in general, nor are the equations generic with respect to their Newton polyhedra. Thus, such a straightforward way to count critical points would be irrelevant.

Computability Questions Since Euler obstructions of polyhedra may be negative, many statements and computations above involve subtraction of polyhedra. The difference of polyhedra $A$ and $B$ is by definition the solution of the equation $B+X=A$. It does not always exist (e.g., the difference of polygons $P$ and $Q$ exists if and only if, for every edge of $Q$, we can find a longer or equal edge of $P$ with the same external normal). If the difference $A-B$ exists, then it is unique for the following reason.

Recall that the support function $A(\cdot):\left(\mathbb{R}^{m}\right)^{*} \rightarrow \mathbb{R} \sqcup\{-\infty\}$ of a polyhedron $A \subset \mathbb{R}^{m}$ is defined by the equality $A(l)=\min _{a \in A} l(a)$. If the difference of support functions $A(\cdot)-B(\cdot)$ is not concave, then the Minkowski difference $A-B$ does not exist; otherwise, $A-B$ can be reconstructed from its support function, which equals $A(\cdot)-B(\cdot)$.

Thus, when computing the Minkowski linear combinations of mixed fiber polyhedra that appear throughout the paper, it is reasonable to encode polyhedra with their support functions. Then Minkowski summation and subtraction is substituted with summation and subtraction of support functions, and mixed fiber polyhedra can be computed by the method of [27] (where the corner locus of the support function of the mixed fiber polyhedron $\operatorname{MP}\left(P_{0}, \ldots, P_{k}\right)$ is computed in terms of the corner loci of the support functions of the arguments $P_{0}, \ldots, P_{k}$ ), or by means of Proposition 2.10 (in [11], this form of the answer is also represented as the mixed volume of certain bounded virtual polyhedra, i.e., the tropical intersection number of the corner loci of their support functions).

For instance, denote the support face of the polyhedron $P \subset \mathbb{R}^{m}$, at which a linear function $\mu \in\left(\mathbb{R}^{m}\right)^{*}$ attains its minimum, by $P^{\mu}$, and discuss the following problem regarding the Newton polyhedron $\mathcal{N} \subset \mathbb{R}^{n}$ of the discriminant that was discussed in the introduction: 
Given the Newton polyhedra $\Delta_{0}, \ldots, \Delta_{l}$ and a linear function $\mu \in\left(\mathbb{R}^{n}\right)^{*}$ that attains its minimum at a vertex of $\mathcal{N}$, compute the coordinates of the vertex $\mathcal{N}^{\mu}$.

We restrict our attention to a coordinate function that we denote by $\lambda$, and compute the coordinate $\mathcal{N}^{\mu}(\lambda)$ as follows:

(1) Since $(P \pm Q)^{\mu}(\lambda)=P^{\mu}(\lambda) \pm Q^{\mu}(\lambda)$, the theorem in the introduction represents $\mathcal{N}^{\mu}(\lambda)$ as a linear combination of values $R^{\mu}(\lambda)$, where $R$ runs over mixed fiber polyhedra of the form $\operatorname{MP}\left(\Delta_{i_{0}}, \ldots, \Delta_{i_{k}}\right)$.

(2) Since the support face of the mixed fiber polyhedron $\operatorname{MP}\left(P_{0}, \ldots, P_{k}\right) \subset \mathbb{R}^{n}$ for a linear function $\mu \in\left(\mathbb{R}^{n}\right)^{*}$ equals the Minkowski sum of mixed fiber polyhedra $\operatorname{MP}\left(P_{0}^{\mu^{\prime}}, \ldots, P_{k}^{\mu^{\prime}}\right)$ over all $\mu^{\prime}$ whose restriction onto $\mathbb{R}^{n}$ equals $\mu$ (see [23] or [13]), we can represent $\operatorname{MP}\left(\Delta_{i_{0}}, \ldots, \Delta_{i_{k}}\right)^{\mu}(\lambda)$ as the sum of $\operatorname{MP}\left(\Delta_{i_{0}}^{\mu^{\prime}}, \ldots, \Delta_{i_{k}}^{\mu^{\prime}}\right)(\lambda)$.

(3) The latter value can be computed by Proposition 2.10 .

In [6], the same problem (of finding the support vertex of a given linear function) for the Newton polyhedron of the discriminant $D_{A}$ is solved in another way, which has two advantages: it is positive (i.e., the algorithm is based on formulas that do not involve subtraction), and it works for tropicalizations of discriminant sets of higher codimension (i.e., when $A$ is dual defect). Thus, it would be useful to generalize the technique of [6] to our setting.

Acknowledgements I am grateful to Askold Khovanskii, Yutaka Matsui, Kiyoshi Takeuchi, Pedro González-Pérez, and Semion Tregub for their fruitful discussions.

\section{References}

1. Bernstein, D.N.: The number of roots of a system of equations. Funct. Anal. Appl. 9(3), 183-185 (1975)

2. Billera, L.J., Sturmfels, B.: Fiber polytopes. Ann. Math. (2) 135(3), 527-549 (1992)

3. Cattani, E., Dickenstein, A., Sturmfels, B.: Rational hypergeometric functions. Compos. Math. 128, 217-240 (2001)

4. Curran, R., Cattani, E.: Restriction of A-discriminants and dual defect toric varieties. J. Symb. Comput. 42, 115-135 (2007)

5. Di Rocco, S.: Projective duality of toric manifolds and defect polytopes. Proc. Lond. Math. Soc. (3) 93(1), 85-104 (2006)

6. Dickenstein, A., Feichtner, E.M., Sturmfels, B.: Tropical discriminants. J. Am. Math. Soc. 20, 11111133 (2007)

7. Ernström, L.: A Plücker formula for singular projective varieties. Commun. Algebra 25, 2897-2901 (1997)

8. Esterov, A.: Indices of 1-forms, resultants, and Newton polyhedra. Russ. Math. Surv. 60(2), 352-353 (2005)

9. Esterov, A.: Indices of 1-forms, intersection indices, and Newton polyhedra. Sb. Math. 197(7), 10851108 (2006)

10. Esterov, A.: Determinantal singularities and Newton polytopes. Proc. Steklov Inst. 259, 16-34 (2007)

11. Esterov, A.: On the existence of mixed fiber bodies. Mosc. Math. J. 8(3), 433-442 (2008)

12. Esterov, A.: Determinantal singularities and Newton polyhedra. arXiv:0906.5097

13. Esterov, A., Khovanskii, A.G.: Elimination theory and Newton polytopes. Funct. Anal. Math. 2(1) (2008) 
14. Gelfand, I.M., Kapranov, M.M., Zelevinsky, A.V.: Discriminants, Resultants, and Multidimensional Determinants. Birkhäuser, Boston (1994)

15. Gonzalez-Perez, P.D.: Singularites quasi-ordinaires toriques et polyedre de Newton du discriminant. Can. J. Math. 52(2), 348-368 (2000)

16. Hall, M.: Combinatorial Theory, 2nd edn. Wiley, New York (1986)

17. Karpenkov, O.: Completely empty pyramids on integer lattices and two-dimensional faces of multidimensional continued fractions. Monatsh. Math. 152(3), 217-249 (2007)

18. Kazarnovskii, B.: c-fans and Newton polyhedra of algebraic varieties. Izv. RAN. Ser. Mat. 67(3), 23-44 (2003)

19. Khovanskii, A.G.: Newton polyhedra and the genus of complete intersections. Funct. Anal. Appl. 12, 38-46 (1978)

20. Matsui, Y., Takeuchi, K.: A geometric degree formula for A-discriminants and Euler obstructions of toric varieties. arXiv:0807.3163

21. Matsui, Y., Takeuchi, K.: Milnor fibers over singular toric varieties and nearby cycle sheaves. arXiv:0809.3148

22. McDonald, J.: Fractional power series solutions for systems of equations. Discrete Comput. Geom. 27, 501-529 (2002)

23. McMullen, P.: Mixed fibre polytopes. Discrete Comput. Geom. 32, 521-532 (2004)

24. Oka, M.: Principal zeta-function of non-degenerate complete intersection singularity. J. Fac. Sci. Univ. Tokyo 37, 11-32 (1990)

25. Sturmfels, B.: On the Newton polytope of the resultant. J. Algebraic Comb. 3(2), 207-236 (1994)

26. Sturmfels, B., Tevelev, J.: Elimination theory for tropical varieties. Math. Res. Lett. 15, 543-562 (2008)

27. Sturmfels, B., Yu, J.: Tropical implicitization and mixed fiber polytopes. In: Software for Algebraic Geometry. The IMA Volumes in Mathematics and its Applications, vol. 148, pp. 111-131. Springer, New York (2008)

28. Tevelev, E.: Compactifications of subvarieties of tori. Am. J. Math. 129(4), 1087-1104 (2007)

29. Varchenko, A.N.: Zeta-function of monodromy and Newton's diagram. Invent. Math. 37, 253-262 (1976) 